\title{
The Influence of Flexural Stiffness on the Performance and Preference of Bendable Stylus Interfaces
}

\author{
by
}

\author{
Alfrancis Guerrero
}

A thesis submitted to the Faculty of Graduate and Postdoctoral Affairs in partial fulfillment of the requirements for the degree of

\author{
Master of Applied Science \\ in \\ Human Computer Interaction \\ Carleton University \\ Ottawa, Ontario \\ (C) 2020, Alfrancis Guerrero
}




\begin{abstract}
Flexible sensing styluses deliver additional degrees of input for pen-based interaction, yet no research on bendable styluses has looked at the influence of stiffness on performance or its integration with creative digital applications. We conducted two experiments that evaluated the influence of flexural stiffness on users' performance. To address this, we designed HyperBrush a flexible sensing stylus with interchangeable flexible components. We assessed the performance of different flexibilities on a bend menu technique. While bend input in styluses can perform similarly to pressure input, researchers only have measured stationary input. We furthered this discussion by evaluating performance of simultaneous bend and $\mathrm{X}-\mathrm{Y}$ pen movement. We conducted a third experiment that investigated how HyperBrush can be a beneficial tool to support users' creativity for digital drawing. We concluded that different flexibilities can pose their own unique advantages analogous to an artist's assortment of paintbrushes.
\end{abstract}




\section{Acknowledgements}

For these challenging past two years, I would like to thank my supervisors Dr. Audrey Girouard and Dr. Thomas Pietrzak for providing their endless guidance and support. If it wasn't for their exceptional experience, and knowledge, I would have not been able to reach this milestone today.

I would also like to give tribute to everyone from the Creative Interaction Labs. I am grateful for being part of a diverse lab culture with driven, enthusiastic, friendly, and welcoming students who shared great interest in their own research and as well as my own. I owe it to Alex Eady for influencing me to peruse a master's degree as well as giving me the opportunity to work alongside with. Yet it is sad to leave such an amazing academic community, everyone has taught me the practical and technical skills to enter the work force and to dominate my future goals and ambitions.

I couldn't have seen myself completing a master's degree without the constant support system from my family and friends. My friends were there for me whenever I needed a break from research as well as being available to study with. I would like to thank Amanda Lam, Heewon Suh, Adam Wang, Andrew Heo, Ramona Sartipi, Chonglu Huang, and Amir Didehkhorshid who demonstrated their encouragement, support, and belief in me every single day. They truly are great friends that I would not ever take for granted. My family supported me with their unconditional love, encouragement, and by always telling me that good things come with hard work and that greatness is right around the corner. 
Lastly, I specifically would like to thank my significant other Casey Wong, as she was there for me physically, emotionally, and mentally, throughout the entire journey always providing her love, empathy, support, and most of all her endless faith in me to achieve greatness. 


\section{Table of Contents}

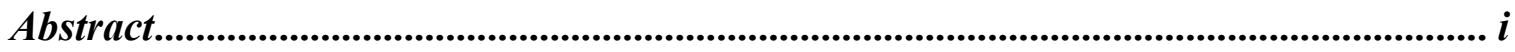

Acknowledgements.................................................................................................. ii

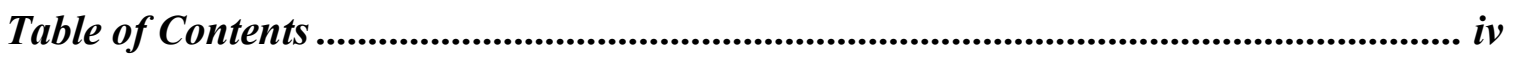

Table of Figures................................................................................................. viii

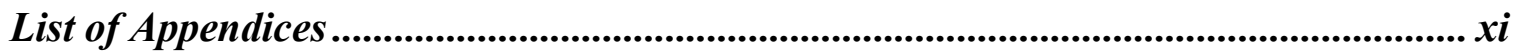

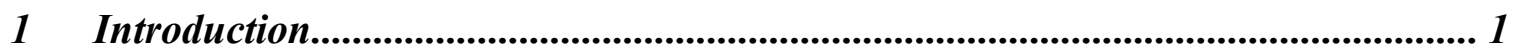

1.1 HyperBrush Prototype.........................................................................................................2

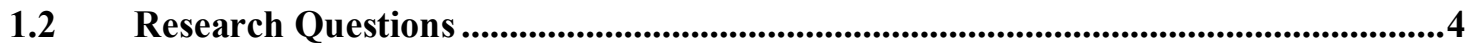

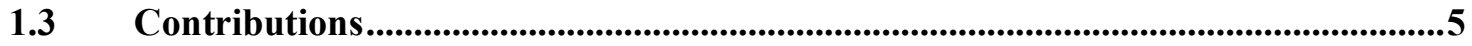

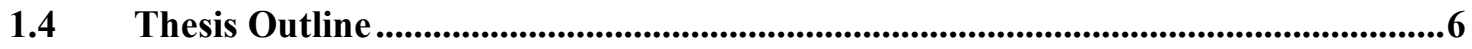

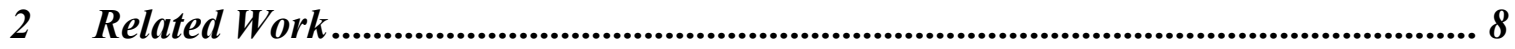

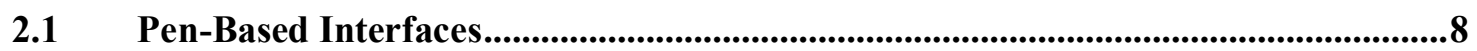

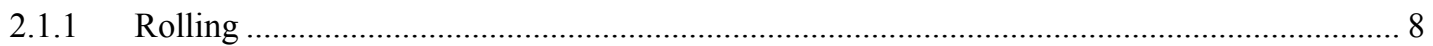

2.1.2 Tilt

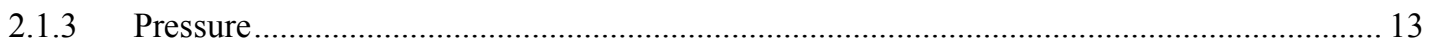

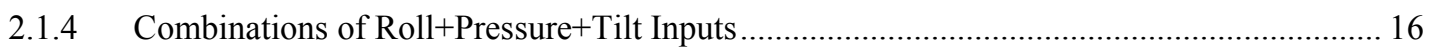

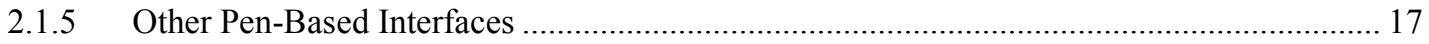

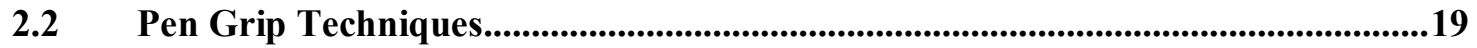

2.3 Pen-Based Menu Designs...................................................................................................21

2.4 Deformable User Interfaces........................................................................................24 
2.4.1 Measuring Flexibility......

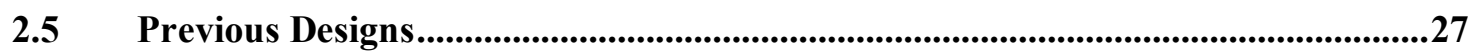

3 Apparatus Construction \& Design Process............................................................ 29

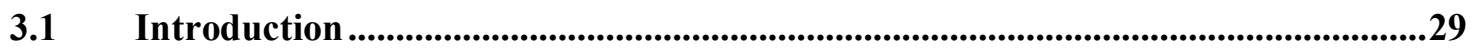

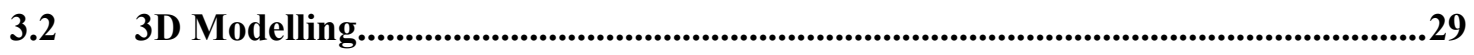

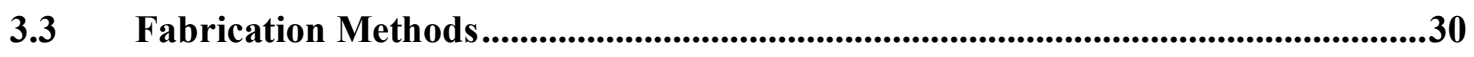

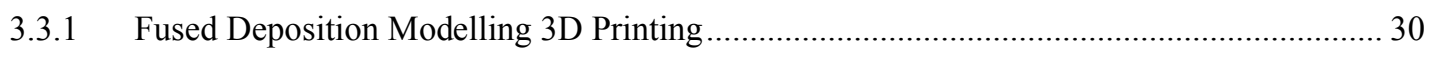

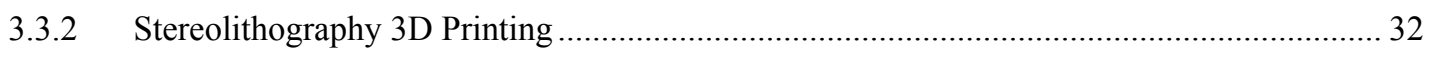

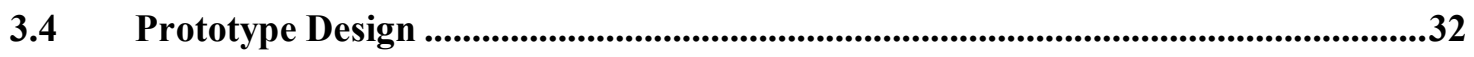

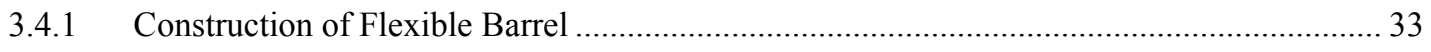

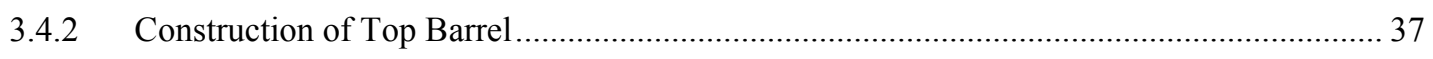

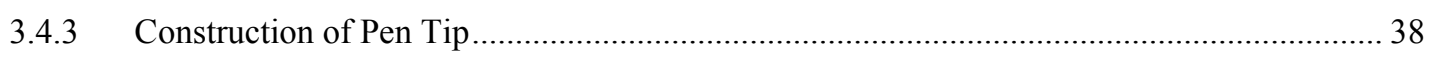

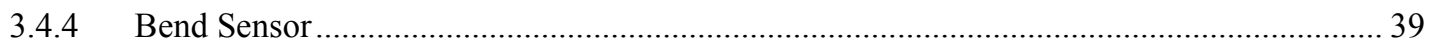

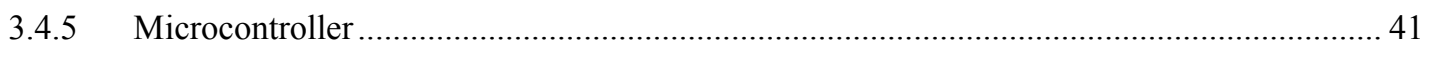

4 Study 1: Bend Menu Selection Technique....................................................... 42

$4.1 \quad$ Introduction ........................................................................................................................42

4.2 Methodology .............................................................................................................42

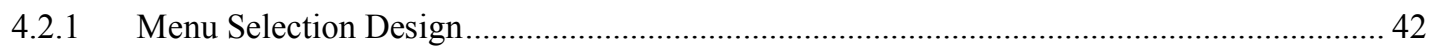

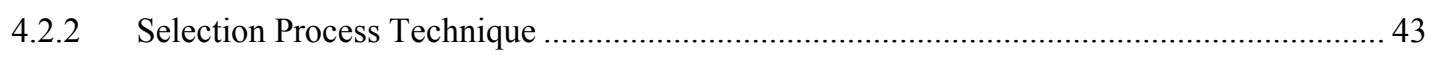

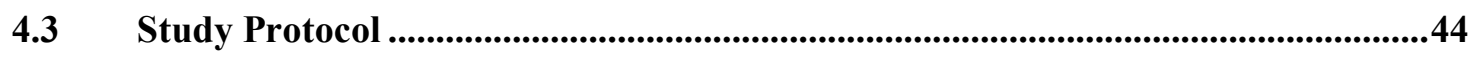

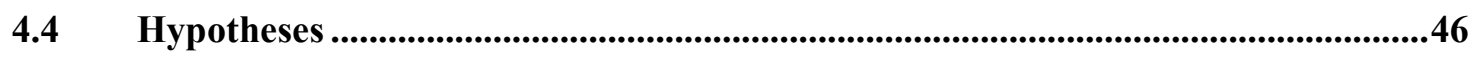

4.5 Participants ........................................................................................................................46

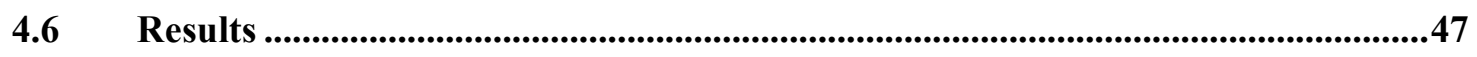




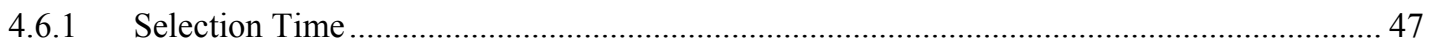

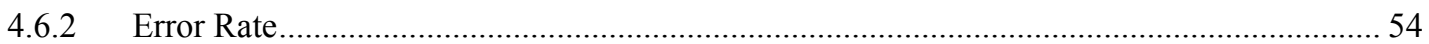

$4.7 \quad$ Flexibility Preference Results...........................................................................................55

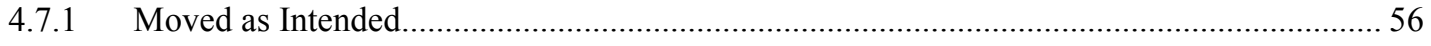

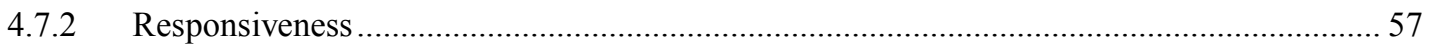

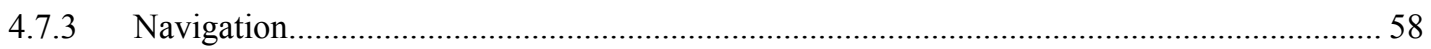

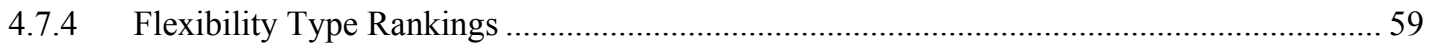

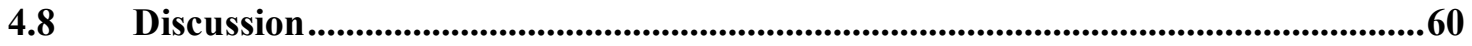

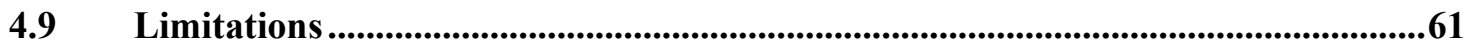

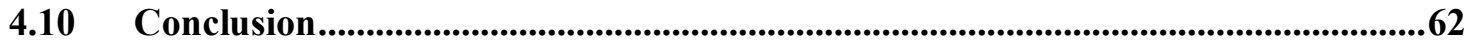

$5 \quad$ Study 2: Brushstroke Width Manipulation ...................................................... 64

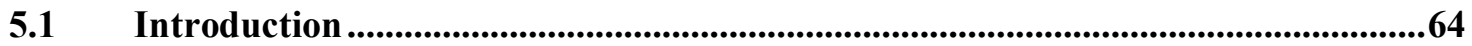

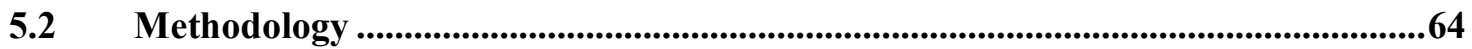

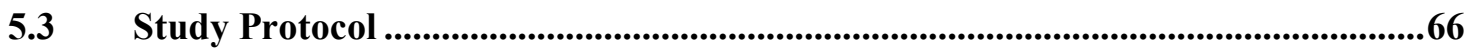

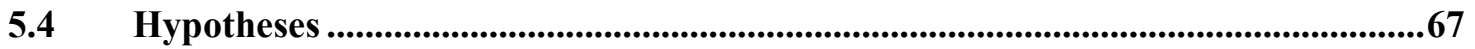

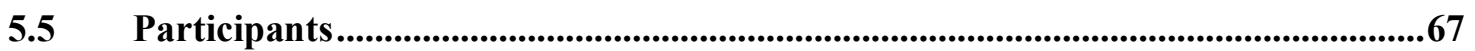

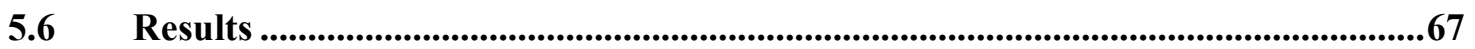

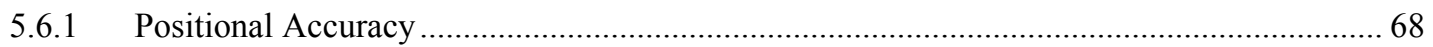

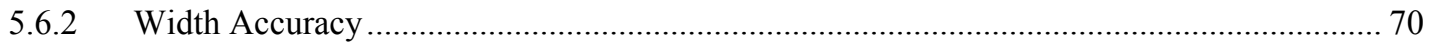

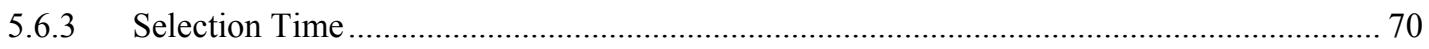

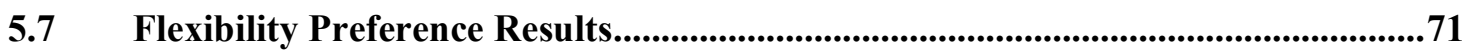

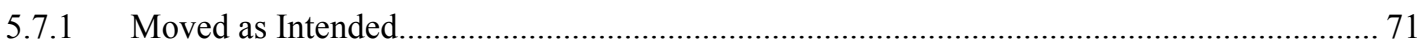

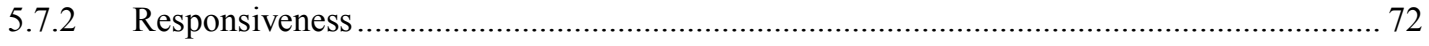

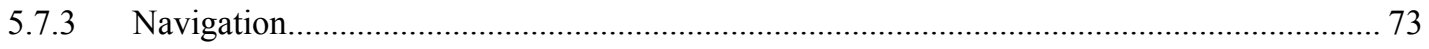




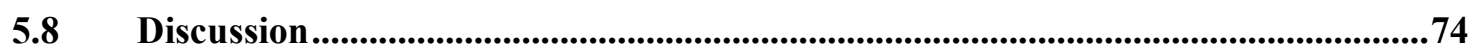

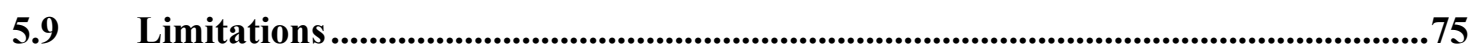

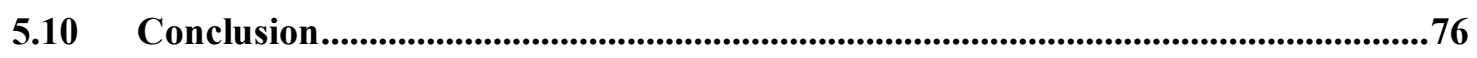

$6 \quad$ Study 3: Creative Digital Drawing Task ...................................................... 77

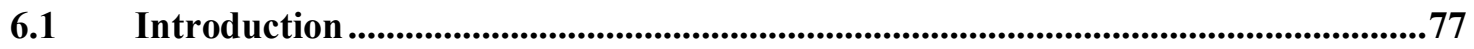

6.2 Methodology ...................................................................................................................77

6.3 Creative Digital Drawing Results .....................................................................................80

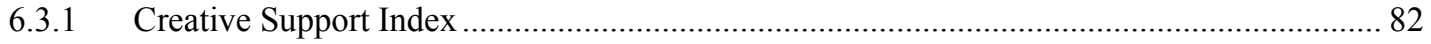

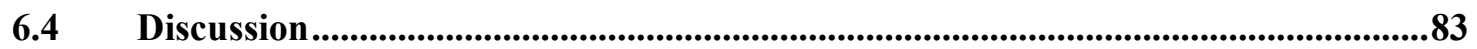

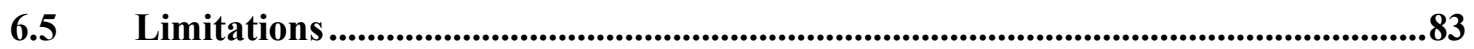

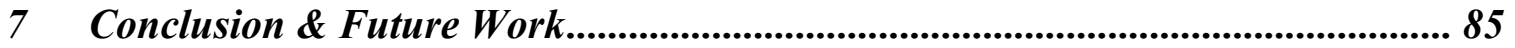

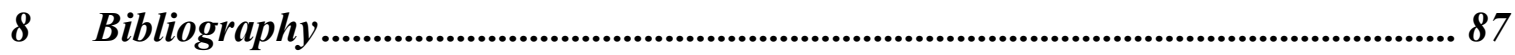




\section{Table of Figures}

Figure 1. Radial Bend Menu selection process. HyperBrush being used to search, select, and confirm selection with using both absolute and rotational bend inputs.

Figure 2. Brushstroke width manipulation study with static and dynamic target patterns. HyperBrush being used to match the target path and width size indicated in blue.

Figure 3. Free-form Digital Drawing Application used in Study 2. HyperBrush being used to draw a landscape by manipulating the size and color of the brushstroke with bending the device.

Figure 4. HyperBrush used for the second study with BendLabs bend sensor and Arduino Mega

Microcontroller.

Figure 5. Collage of iterations of prototypes made from a uniform single material (red thermoplastic urethane) to a multi-material design (blue, black, and transparent photopolymer resin).

Figure 6. Early design of a TPU flexible stylus prototype that was printed horizontally with PVA water soluble support material. (Top) Prototype split into two held together with blue electrical tape. (Bottom) Thicker prototype that did not split but difficult to bend.

Figure 7. To scale cross-section models of both experiments stylus designs. Experiment 1 (Top), with a concave upper barrel and pseudo-button that suggests orientation of the users' grip. Experiment 2

HyperBrush (Bottom) features additional inner screws that improve durability and modularity.

Figure 8. Top view of both final design's styluses separated apart with screw design.

Figure 9. Front cross section view of the five $3 D$ printed flexible barrels with flexural stiffness measurement not including the bend sensor inside. The medium* flexibility was used for the second study.

Figure 10. Experimental setup for a beam deflection test. HyperBrush being suspended at the fixed end and a load force applied at the other end of the flexible barrel (orange). 35

Figure 11. HyperBrush designed for menu selection experiment with pseudo-button (Blue) and the HyperBrush design for the brushstroke experiment (Black). Circled in Red is the pseudo-button. 37

Figure 12. BendLabs Inc. two axes soft angular displacement sensor. 39 
Figure 13. - Left. Top cross section view showing the paired capacitors for the X and Y planes. Right.

Perspective diagram of the barrel and its bending axis [52]. 40

Figure 14. Radial Menu designs. Left: 4-items, Middle: 8-items, Right: 12 items. 43

Figure 15. Menu selection process of the cursor (pink circle) to target (green item) shown in the medium size menu. 1. Cursor starting in the neutral zone. 2. Moving into selection zone (highlighted in red). 3.

Navigating to target item in green. 4. Exiting on the outside radius of the target item to confirm selection.

Figure 16. Average selection time and error rate for repetitions with $95 \%$ CI. 49

Figure 17. Average selection time (Left) and error rate (Right) with 95\% CI for each menu type. 51

Figure 18. Selection Time and Error Rate for items within the Small Menu. 53

Figure 19. Selection Time and Error Rate for items within the Medium Menu. 54

Figure 20. Selection Time and Error Rate for items within the Large Menu. 54

Figure 21 - Likert scale responses on the question, "Did the cursor moved as intended?" 56

Figure 22 - Likert scale responses on the question, "How responsive was our device?" 57

Figure 23 - Likert scale responses on the question, "Are you able to navigate to the target?" 58

Figure 24. Total participants ranking from most $\left(1^{\text {st }}\right)$ to least $\left(4^{\text {th }}\right)$ preferred flexibility input type. 59

Figure 25. Brushstroke width manipulation task with the static (top) and dynamic (bottom) target patterns. Illustration of a user matching their path (dark blue) to the target path (light blue). The cursor (Black rectangle with a circle in the centre) indicates the current position and width size. 65

Figure 26. Positional Accuracy - Participants average X-Y distance from the target position for the three flexibility conditions and the pressure condition. 68

Figure 27. Positional Accuracy - Participants average $X-Y$ distance from the target position for both target type. 69

Figure 28. Average completion times for each direction with 95\% CI. 71

Figure 29. Likert scale responses on the question, "Did the cursor moved as intended?" 71

Figure 30. Likert scale responses on the question, "How responsive was our device?" 72

Figure 31. Likert scale responses on the question, "Are you able to navigate to the target?" 73

Figure 32. Summary of participant rankings from most $\left(1^{\text {st }}\right)$ to least $\left(3^{\text {rd }}\right)$ preferred flexibility input type. 73 
Figure 33. Drawing Application with HyperBrush.

Figure 34. Menu buttons for Brushstroke Size Modifier (Active) and Gradient Toggle with Modifier

(Inactive). 78

Figure 35. Interface for the Colour Modifier Toolbar.

Figure 36. Menu shortcut buttons for black, eraser, and clear.

Figure 37 - Collage of participants' drawings of a landscape or cityscape from using both styluses.

Figure 38. Example of P12 drawing colorful gradient clouds with using repetitive circular motions while bending and rotating the HyperBrush.

Figure 39. A. Perceived average Likert scale ratings with SD for each dimension of creativity and B. Calculated Creative Support Index (CSI) scores with SD for our HyperBrush and the baseline condition MS Pressure Pen. 


\section{List of Appendices}

Appendix A............................................................ 97

A.1 Consent Form................................................... 97

Appendix B.......................................................... 96

B.1 Study $1 \& 2$ Demographics Questionnaire..................................99

B.2 Between Task Questionnaire...................................... 103

B.3 Post Questionnaire............................................... 104

B.4 Creative Survey Index Questionnaire...............................106

Appendix C. .......................................................... 108

C.1 Participant Drawings with HyperBrush..................................... 108

C.2 Participant Drawings with Pressure Pen............................... 111 


\section{Introduction}

Pen-based interfaces are used in various digital applications such as sketching, drawing, writing, and 3D modelling. Research prototypes and consumer electronic products can feature additional degrees of freedom to provide a larger input vocabulary: tip pressure [45, 46], tilting in a direction [56], rolling of with the fingers [8], or tapping the barrel have exhibited positive results $[21,53]$. One can use these extra degrees of freedom to create variable brushstroke sizes $[5,18]$, manipulating the orientation and positioning of models, selecting items [20], and operating menus [6,63]. The initial goal behind having additional inputs is to increase productivity, efficiency, and promote creativity [5]. Such research has attempted to simulate the experience of using non-digital tools such as the pencil [14] or paintbrush $[5,18]$. While these inputs can be of great benefit to stylus-based applications, there are still some limitations posed to each. For example, pen pressure input can be difficult to control while decreasing in pressure opposed to increasing in pressure [46, 64].

Research has also shown that pen tilt can be susceptible to hand occlusion making items in a radial menu difficult to reach or select [57]. Fellion et al. first introduced the bendable input in a stylus. Their study compared the performance of controlling the width of a brushstroke using bend and pressure input, which resulted in similar performances. While their studies showed promising results, pen movement was not evaluated which is an essential input for pen-based interfaces. Therefore, we decided to further this field of research by measuring pen movement and additionally measure the influences of flexural stiffness on users' performance and preference. Flexible devices are on the rise of much research and have shown to be a viable form of interaction $[18,30]$. When studying flexible 
devices, it is important to consider how flexible the device should be. With the advancements of $3 \mathrm{D}$ printing, we explore such flexibility by changing the physical properties in our devices design.

\subsection{HyperBrush Prototype}

We present HyperBrush, a flexible stylus capable of sensing bend input to be functioned as two additional degrees of freedom for pen-based interfaces. We derived the name HyperBrush inspired from feedback from participants that referred to our device as paintbrush like and "hyper" derived from "hyperextend" to extend or bend beyond limits. Our HyperBrush measures two degrees of bend input: absolute bend - the amount of bend being applied — and rotational bend — the azimuth angle relative to the barrel of the stylus.

We designed HyperBrush with interchangeable flexible components that vary in flexural stiffness. We further this field of research by evaluating the usability of bend while dynamically moving the stylus and compare creative performance and user's preference to pressure input. Additionally, we assessed the usability of bend input to select items in marking menus. We also measured the effects of various levels of flexural stiffness.

Figure 1, Figure 2, and Figure 3 illustrates our HyperBrush being used for the three studies. Our first study couples both absolute bend and rotational bend to navigate and select items in a radial menu while study 2 and 3 only incorporate absolute bend. Figure $1-2 \& 3$ demonstrates rotational bend is being used to search through items in the circular menu. While Figure 1-4 shows absolute bend being used to confirm selection by moving 
the cursor a greater distance from the center of the origin.
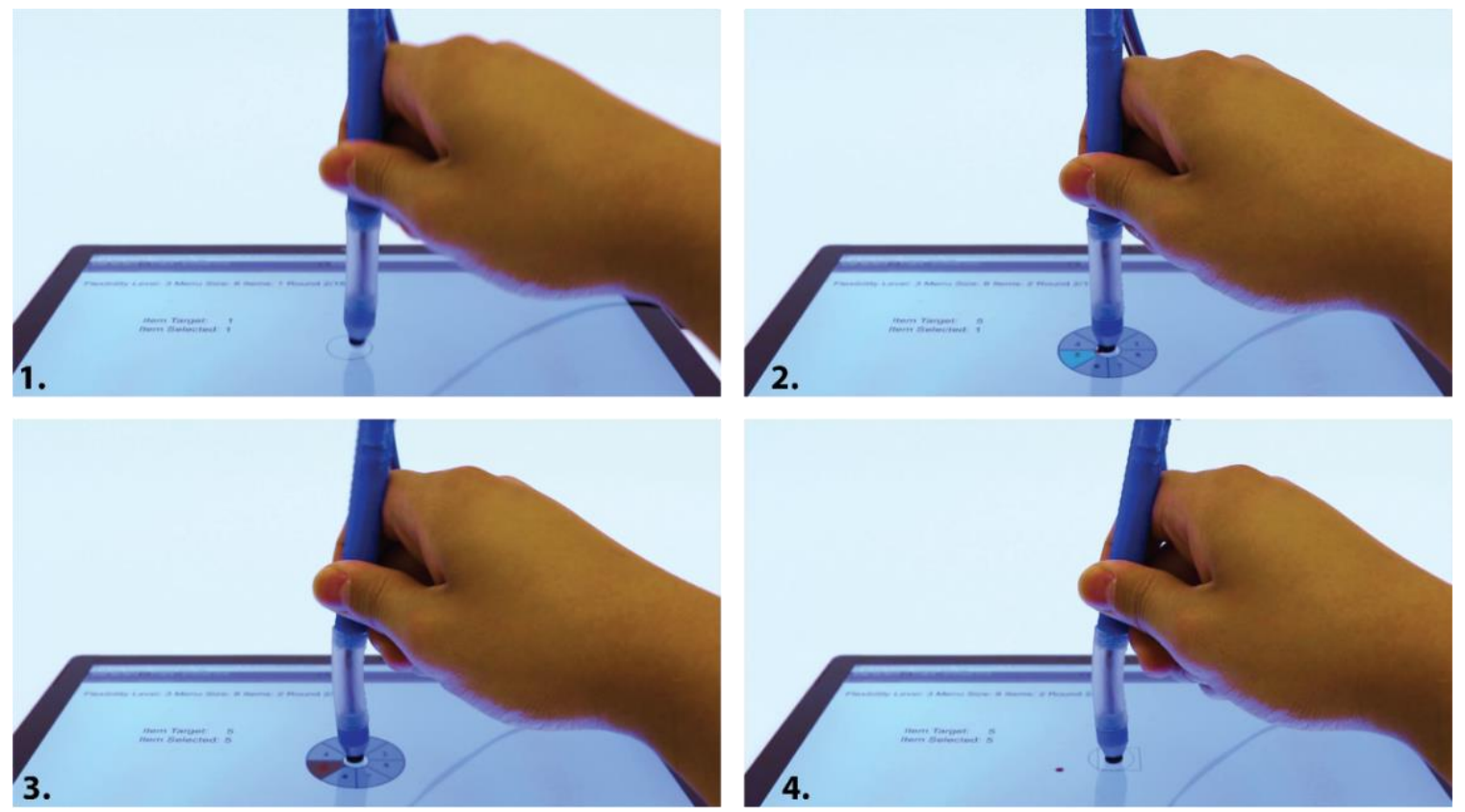

Figure 1. Radial Bend Menu selection process. HyperBrush being used to search, select, and confirm selection with using both absolute and rotational bend inputs.
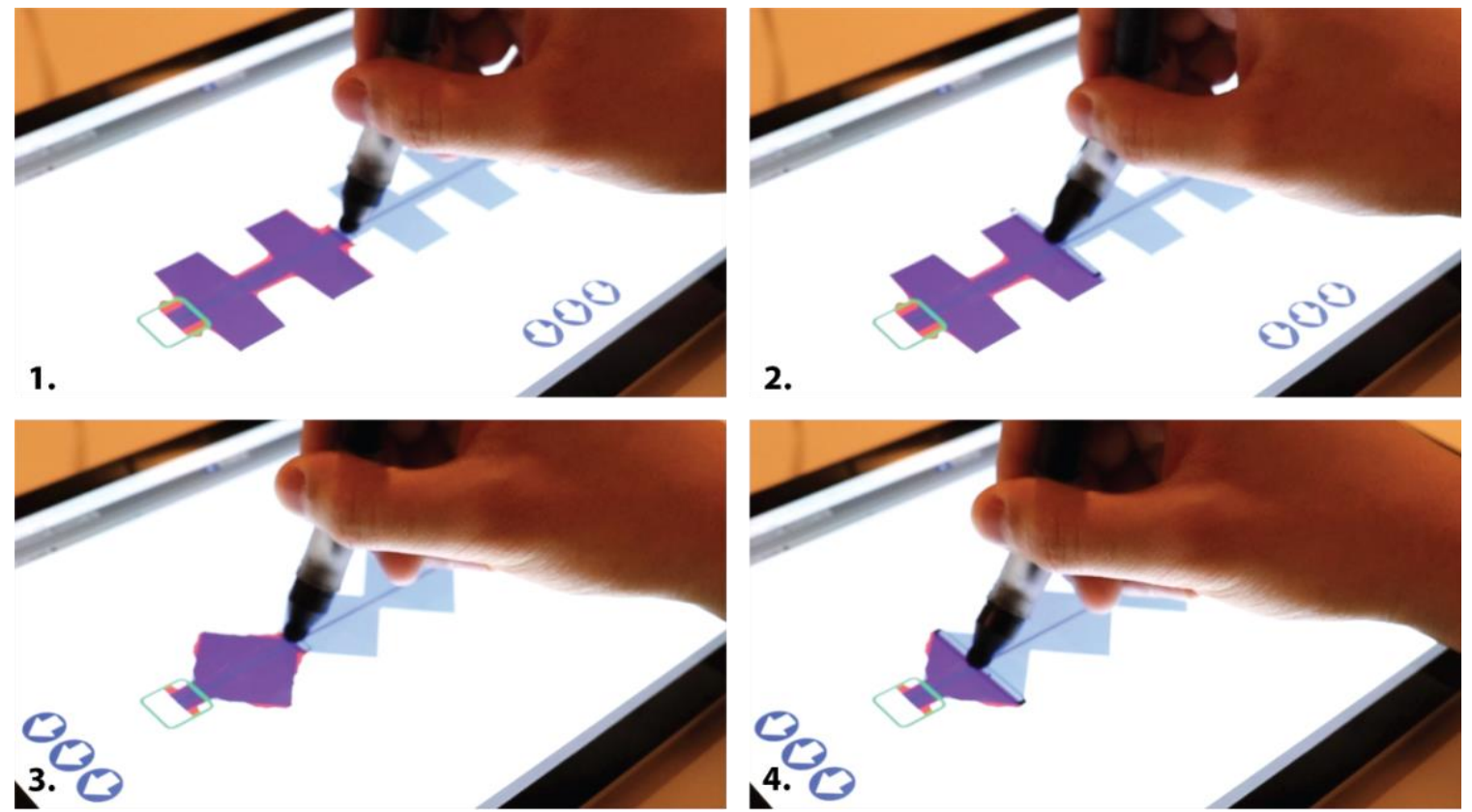

Figure 2. Brushstroke width manipulation study with static and dynamic target patterns. HyperBrush being used to match the target path and width size indicated in blue. 

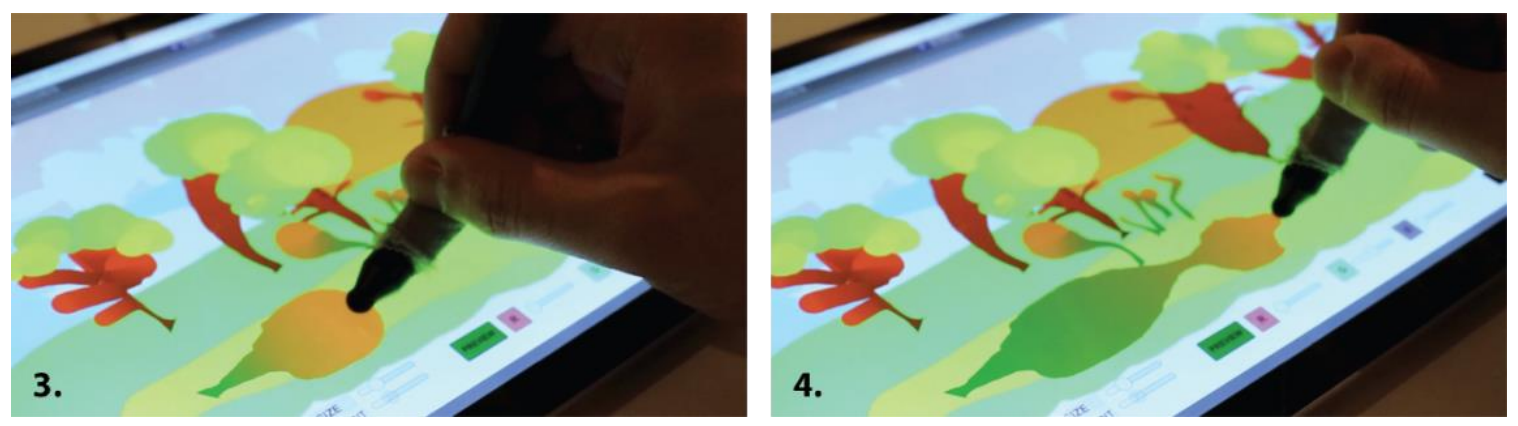

Figure 3. Free-form Digital Drawing Application used in Study 2. HyperBrush being used to draw a landscape by manipulating the size and color of the brushstroke with bending the device.
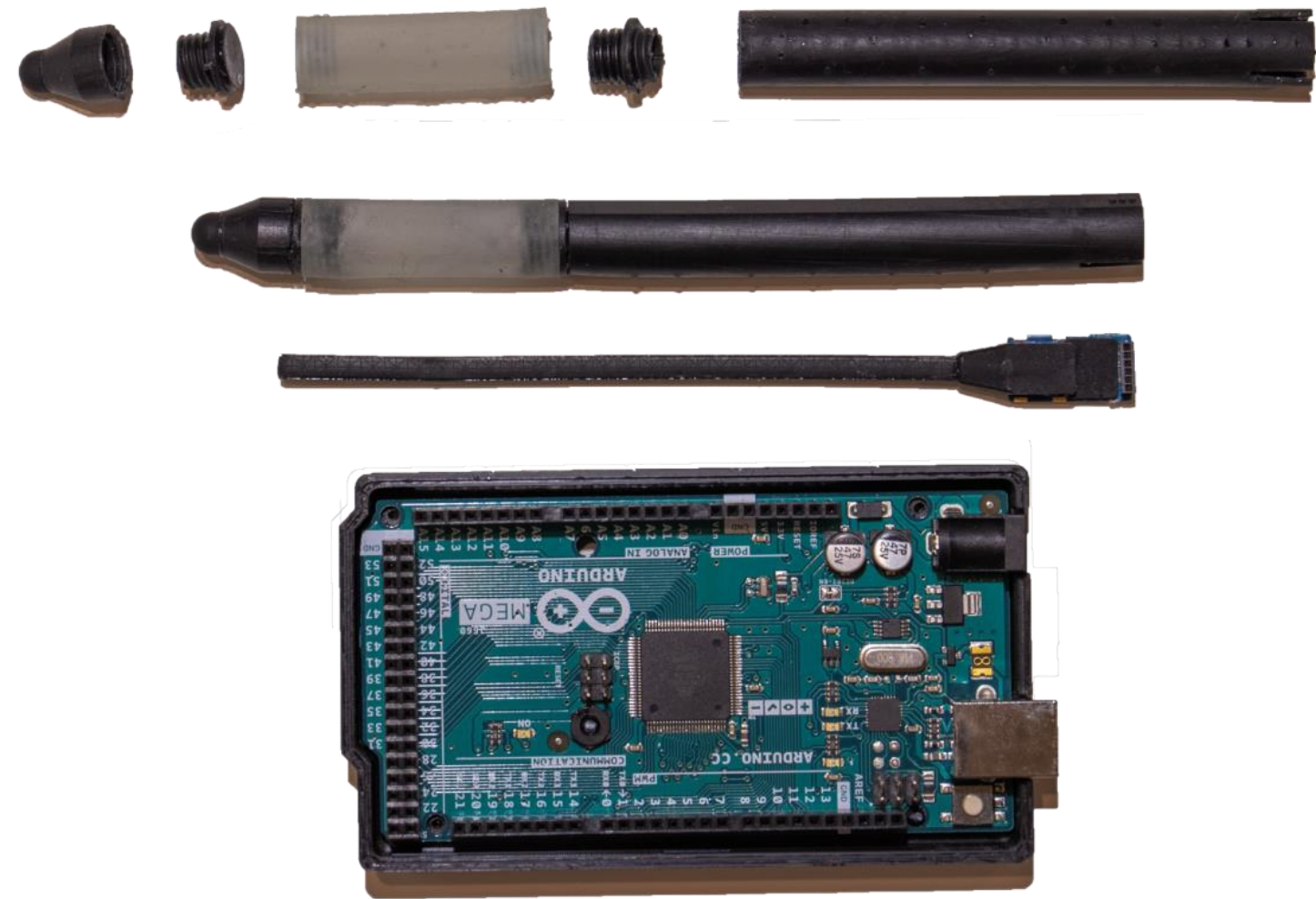

Figure 4. HyperBrush used for the second study with BendLabs bend sensor and Arduino Mega Microcontroller.

\subsection{Research Questions}

R1) What is the best performing and most preferable flexural stiffness for a flexible stylus device? 
R2) Can bend input perform similarly to our baseline pressure input during brushstroke width manipulation and pen movement?

R3) Can our HyperBrush support users' creativity better than our baseline pressure input device?

\subsection{Contributions}

We developed two HyperBrush prototypes one for each study and evaluated the influence of flexural stiffness being the main objective. Below we outline our contributions as the first attempts to design a modular flexible stylus, analyze the influences of flexural stiffness, and measure the creative supportiveness of a flexible stylus.

\section{C1: Design and fabrication of a modular flexible digital stylus with bend input} capabilities. Our first contribution is our design and construction of a modular 3D printed bendable stylus. We designed HyperBrush to be able to interchange flexible barrels to accommodate a range of flexural stiffnesses. We classified each flexibility type as a ratio of force applied per unit distance displaced. We walk through our iteration process, our past prototypes leading up to our latest design, our limitations, and previous designs such as FlexStylus [18] in Chapter 3.

\section{C2: Evaluation of flexural stiffness on bend input during menu selection. For our} second contribution, we performed a study on how flexural stiffness influence user's performance and preference during bend menu selection. We develop our own variation of this technique that was previously introduced by Fellion et. al [18]. 


\section{C3: Evaluation of flexural stiffness on simultaneous control of bend and positional}

input during brushstroke width manipulation. Fellion et. al compared pressure input to their FlexStylus's flexible input for stationary brushstroke manipulation and resulted in similar performance between the two [18]. Our goal was to evaluate the performance of flexural stiffness while simultaneously controlling pen movement. We used pen pressure as a baseline to see how our results compared to the baseline and more established pen pressure input technique.

\section{C4: Comparison of bend and pressure input on supporting creativity in digital}

drawing applications. As mentioned before, additional stylus input can enhance and support the user's experience. We incorporated a free form digital drawing task and measured users perceived creative support for bend and pressure. We followed a methodology previously done by Aslan et al. that also compared the supportiveness from their multimodal pen and a baseline pen device [13]. We emphasize the importance of C3 and $\mathrm{C} 4$ as these studies evaluate HyperBrush in a more realistic digital drawing context.

\subsection{Thesis Outline}

Chapter 1 discusses our motivation and research goals, following we discuss areas of research such as pen input techniques, menu interfaces, gripping techniques, and flexible interfaces in Chapter 2. Chapter 3 outlines HyperBrush's iterative prototypes, our method of interchangeable components, and how our prototype differs from previous designs as well as the sensing technique. Chapter 4 explains our first study that evaluated the effects 
of flexural stiffness on a bend menu selection technique. We also report our findings from our survey questionnaire and data analysis in Chapter 4 . Chapter 5 covers our second study in which we study the effects of simultaneously controls bend and pen position. Following, Chapter 5 also reports the data analysis and flexibility ranking preferences. Chapter 6 discusses our third study that evaluated how HyperBrush can be a tool to support creativity. Chapter 6 also entails the qualitative findings, feedback, and discusses patterns and trends found in participants illustrations. Lastly, Chapter 7 discusses the future work and conclusion of this thesis. 


\section{Related Work}

Our research was built on different areas of stylus interfaces, grip techniques, and designs. We explored prior work that has evaluated popular stylus-based input techniques such as pressure, tilt, rotation. We discussed research on pen gripping techniques that suggested contextually relevant tools and mode switching. Lastly, we examined different pen-based menu stylus, marking menu techniques, and deformable based interfaces.

\subsection{Pen-Based Interfaces}

Pen input is primarily focused on tracking the $\mathrm{X}-\mathrm{Y}$ position of the pen tip on screen with additional degree of inputs such as sensing pressure, tilt, or rotation [8]. In this section, we looked at previous research that explored the usability of additional degrees of inputs that extends the inputs available on pen-based devices.

Prior research explored various forms of auxiliary input such as rolling [8,21, 23], pressure $[11,17,45,46]$, tilt $[21,56,60]$, and bend $[17,18]$ that go beyond tracking the $\mathrm{X}-\mathrm{Y}$ position of the pen tip on the screen $[8,61]$. Pen rolling utilizes the $3 \mathrm{D}$ rotation of the pen along its longitudinal axis as an input [8]. Tilt is measured from the 3D orientation of the pen while the pen tip acting as the centre axis [57]. Lastly, pressure input is the amount of force applied at the stylus tip on the surface of the paired touch screen [45]. This section describes previous implementations and techniques involved with the three types of auxiliary inputs.

\subsubsection{Rolling}

Researchers have been exploring different pen rolling techniques $[8,21,23,50]$. Hinckley et al. [8] also proposed various pen rolling applications such as zooming, scrolling, rotating 
2D objects, and rotating the tangent of an anchor point for drawing based applications. They implemented selection tasks similar to a radial menu where each item was separated by $45^{\circ}$ rolling angles. This was utilized so that they could have users quickly select common tools such as an eraser, a brush, a pen, and a flood-fill tool.

Hinckley et al. conducted a study evaluating pen rolling behaviours and concluded 1) A distinction between accidental and intentional pen rolling and 2) A comfortable rolling range of degrees [8]. To determine the distinction between accidental and intentional rolling, they ran an experiment consisting of common stylus tasks measuring rolling angle and rolling speed. Rolling angle is defined as amount of rotation from start to end and rolling speed is how fast the user rolls the pen in an increase of delta time. From their experiment, they identified pen incidental pen rolling when it falls within the rolling speed range of $[-30,30]$ degrees/s and rolling angle of $[-10,10]$ degrees. They concluded that anything outside these ranges would be considered as intentional pen rolling. Their second experiment to determine a comfortable rolling range, had participants roll the cursor to the target degree location. From their results, they concluded that the most accurate and comfortable rolling range was between -90 and 90 degrees. Anything outside these bounds resulted in higher completion times and error rates, and overall, it was not desirable or comfortable to execute from participants.

Hinckley et al. observed that it is easier to roll in one direction than the other. For righthanded users, it is easier to roll the pen clockwise than rolling counterclockwise, and reversed for left-handed users [23]. They implemented a roll to undo where the user triggers 
the undo action by rapidly rolling the pen along its longitudinal axis in either direction. Following undo to roll motion, tapping the barrel then repeatedly invokes undo because pen rolling repeatedly would be found undesired and slow. However, their gesture removes the need of the user finding the undo button in an on-screen menu. From their evaluation, they found that many of their users experienced false positives while performing other actions during the study. They also combined rolling with touch selection to enable the manipulation of object orientation or to cycle through application layers. Feedback from users showed fewer problems than rolling to undo, however, the tapered shape of their pen used made it difficult for users to roll completely without unintended tumbling. They proposed that a uniformly cylindrical barrel in a stylus should be ideal for pen rolling techniques for reducing false positives.

In summary, pen rolling has shown that it can be effective for quick selection between tools, sliders, and scroll bars [8]. However, rolling can be restricted to the comfort of the user's grip and therefore rolling the pen more than 180 degrees can result in difficulties and become more error-prone [23]. Hinckley et al. also recommended a uniform cylindrical design of the stylus to improve user comfort during rolling [23].

\subsubsection{Tilt}

Pen tilt is different than pressure and rolling because tilt can be visually indicated by looking at the angle of the pens barrel relative to the surface of the screen. It is much more difficult to visualize a variable of pressure or the angle at which you are rolling a pen [60]. Xiangshi et al. [60] conducted two experiments on evaluating the human ability to control and perform selection tasks with pen tilt. Their first experiment assessed how well a user 
can acquire a target using pen tilt. Their tilt acquiring experiment had users tilt the angle of a pen to the target tilt angle then touch to land the pen tip on the tablet surface. After contact, the pen tilt angle was indicated on the interface and the users were to guide the cursor to the target angle. They tested various target angular widths of $5^{\circ}, 10^{\circ}, 20^{\circ}, 30^{\circ}$ and $90^{\circ}$ was located directly perpendicular to the tablet surface. They calibrated the pens angle at $0^{\circ}$ when lying flat on the surface of the tablet. They observed that when target angles were obstructed by the hand it made it difficult for users to select it resulting in higher completion times. They also measured the Number of Crossings (NC), which is how many times the user enters then leaves the targeted area. They noticed that the most amount of NCs occurred at the angle when the pen was at its most perpendicular to the screen's surface. They defined tilt comfort zones for pen tilt to range between $30^{\circ}$ to $150^{\circ}$ with an angular width of $30^{\circ}$ and $100^{\circ}$ to $150^{\circ}$ with an angular width of $20^{\circ}$. For these ranges their measured selection time averaged less than 1 second, error rate was lower than $10 \%$ and $\mathrm{NC}$ was less than 1 . Angular widths below $10^{\circ}$ resulted in unacceptable error rates. Most of their participants adjusted their pens angle in air then made fine adjustments after touching the surface. They sought that this was because there was no occlusion happening when tilting in air.

Xiangshi et al. proposed a taxonomy of conceptual interaction techniques that can benefit from using tilt as form of input [60]. They propose a fan menu, that is a radial pie menu divided into segments with a fixed angular width with each segment corresponding to the tilt angle of the pen. They augmented current sliders in GUI such as map navigation, video scrolling, or page scrolling to be manipulated using the displacement of pen tilt. They also 
propose a projection cursor that is shrinking when the pen tilt angle is reaching its perpendicular to the tablet's surface. They suggest that this technique can be effective for specifying an area or provide graphical visual feedback of the tilt angle. They describe how mode switching for drawing applications such as switching between a "hard" or a "soft" paint brush can be invoked by the gesture of a pen tilt. Lastly, they discuss how 3D manipulation of objects can be rotated according to the pen tilt or that objects can be multiplied where the subordinates are created in the direction of the bend.

Another variation of utilizing pen tilt for menu navigation was to also measure the horizontal angle of the pen known as the azimuth. Guozhong et al. takes advantage of this input with the vertical angle to navigate through radial menu items [57]. As opposed to other techniques that used only the vertical angle for item selection, this method allows items to be directly collocated underneath the pens azimuth angle when trying to select it [60]. They used the azimuth angle to navigate through items and the vertical altitude angle to initiate confirmation of selection.

Our first study looked at the effects of flexural stiffness on a menu selection tool. Our selection technique was similar to Tilt Menus selection technique [57]. Rather than the azimuth being used for navigation and tilt angle being used for confirmation, we couple our HyperBrush's rotational bend for navigation and absolute bend for selection confirmation. Furthermore, on our selection technique is explained in chapter 4.2.1 Menu Selection Design. 


\subsubsection{Pressure}

Pressure Widgets investigated the human ability to discriminate discrete levels of pressure [45]. They also considered the impact on visual feedback and what form it should take. Their experimented was a selection task that asked the user to control the linear movement of a small blue circle. The objective for their task was to move the ball into a target highlighted area by controlling the amount of pressure. They provided two forms of visual feedback, Full Visual (FV) - a series of gridded white rectangles outlining the range of ball movement, and Partial Visual (PV) - where there were no gridded white rectangles. They tested four different types of pressure selection methods each was used to confirm selection once the target pressure was acquired by the user. The techniques used; Dwell - held the cursor within the target for 1 second, Quick Release -quickly lifting off of the tablet, Stroke - spatially moving to the right, and Click - a press of the built-in barrel button. Dwell showed most accurate results as the user just had to hold the position and wait for the 1 second mark to confirm selection. Although the most accurate, the trade-off for this interaction is the 1-second delay after successfully navigating to the target. They labelled the Stroke to be the most difficult as feedback from most participants. Participants stated that it was difficult to maintain a consistent pressure value while moving the stylus in the horizontal direction. They also mentioned that this was the most difficult to learn, as participants performed the interaction at different rates when it was intended to be a quick fluid motion. Quick Release resulted in best completion times due to its fast motion during the second motion, as opposed to the $d$ well technique. Their last technique Click showed significant interference with pressure control. Overall, they determined that humans were able to identify between six discrete levels of pressure. 
While selections and actions in Graphical User Interfaces (GUI) are often broken down into two sequential commands [24] i.e., selecting a group of items then proceeding to copying, Pressure Marks, proposed a novel technique that performs both a selection-action command simultaneously by utilizing pressure input [46]. Their interaction established shorter completion times than sequential selection-action patterns. For their study, they classified four signature pressure marks that are indicated by the start and ending pressure level (e.g., Low-Low, Low-High, High-Low, High-High). They ran an exploratory user study that evaluated the latter for straight and lasso stroke types and found that pressure marks can be easily reproduced by users. Their initial observations found that the lasso strokes were more difficult when holding a consistent pressure than the straight strokes. Interestingly, they found that it was easy for their participants to execute Low-High strokes. However, strokes that were High-Low were the most difficult and the least desirable of the four signatures. Their second experiment found that their pressure marks interaction outperformed the existing technique of lasso + pigtal2 defined by Hinckley et al. [22].

Zhou et al. conducted a comparison study between the performances of pressure and tilt input when coupled to cursor position, angle, and scale [64]. For their first study, they mapped pressure and tilt control to control the position of a cursor that moved in a linear direction. They uniformly divided both inputs to $4,6,8$ and 12 discrete pressure and tilt levels. They programmed the on-screen cursor by an increase in pressure or tilt which will move the cursor to the right while a decrease will move the cursor to the left. The user's objective was to navigate to a target using either pressure or tilt and confirm selection with 
a key press when hovering over it. From this study, they found that moving towards the left: decreasing in pressure, resulted in a much higher error rate and completion time when compared to increasing in pressure and moving both directions for tilt. Their other two studies coupled pressure and tilt to controlling the angle on a rotating needle and a circular cursor. They programmed the needle to rotate around a $\left[0^{\circ}, 360^{\circ}\right]$ circle where 0 pressure or a $0^{\circ}$ tilt angle was mapped to $0^{\circ}$ rotation. An increase in pressure and a positive rotation in tilt would increase the angle of the needle. For the last study, an increase in either input would increase the scale of the circular cursor and decreasing vice versa. Interestingly, a decrease in pressure also resulted in significantly higher error rates and completion times.

While pressure input has been shown to be effective in many different pen-based applications such as selection-action commands, marking menus, and digital drawings, there still pose some limitations. Ramos et al. found that humans can only distinguish up to 6 levels of pressure [45]. Research has also found that it was difficult for users to perform tasks that required a decrease in pressure level as it compromised completion time or accuracy [64]. Therefore, within our second study we included a task that focused on measuring the performance of changing (increasing and decreasing) bend and pressure input during linear movement. We were interested in evaluating increasing and decreasing of bend input while moving the stylus position for different flexural stiffnesses. We used pressure input as our baseline input technique to link our bend input. 


\subsubsection{Combinations of Roll+Pressure+Tilt Inputs}

By combining two or more auxiliary inputs, this can expand the input space and introduce new forms of interaction [21]. Hasan et al. refers to their combination technique as a-coord input. They conducted a study that evaluated if users can successfully coordinate two auxiliary input channels simultaneously [21]. Their experiment tested three different $a$-coord techniques Roll + Pressure $(\mathrm{R}+\mathrm{P})$, Tilt + Pressure $(\mathrm{T}+\mathrm{P})$, and Roll + Tilt $(\mathrm{R}+\mathrm{T})$ for a radial menu selection targeting task. The task had the first channel control movement radially and the second channel control movement linearly outwards from the centre. Their results revealed that $T+R$ yielded the best overall results with acceptable completion times and error rates. They stated that rolling a pen within the bounds of +-80 degrees controlled more discrete levels than pressure and tilt. Tilt was found to be good at controlling the radial movement because it did not require the user to transverse through a path to get to its target location. In other words, participants were able to tilt the pen directly to where the target was located as opposed to roll or pressure which 
required participants to transverse to the target. Their experiment evaluated a two-level menu selection that which used two auxiliary input channels. However, the study was not restricted to simultaneously manipulating both at once therefore participants were able to focus on one input channel at a time.

In Hasan et al.'s second study, they evaluated the same a-coord inputs that involves continuous manipulation of both input channels simultaneously [21]. Unlike the first experiment, one channel is for item selection and the other channel is for continuous variable manipulation. This means the user holds the position of the primary channel steadily while manipulating the secondary channel. Their experiment had participants select a slider using pressure or tilt, then manipulate the value of the slider with roll. The $\mathrm{P}+\mathrm{R}$ technique had four sliders placed side by side while the $\mathrm{T}+\mathrm{R}$ was implemented as a FaST Slider which allowed for quick selection and movement [41]. They concluded that holding tilt while rolling was more controllable as opposed to holding a pressure value while rolling. In summary, the combination of tilt + roll was efficient for parametric selection and manipulation tasks and would be a viable augmentation to existing multiparameter input techniques.

\subsubsection{Other Pen-Based Interfaces}

This section highlights some novel pen interfaces that are not focused on the traditional input from rolling, pressure, or tilt to enhance the digital pen user experience. Rather, some 
gain inspiration and try to take affordances from analog tools such as writing on a piece of paper[14], or utilizing the physical features of a crayon [58].

RealPen mimicked the physical sensation of writing on a physical piece of paper with a pencil [14]. They achieve this sensation by the regeneration of the friction induced oscillation and sound. Conventional stylus and pen designs try to enhance other experiences such as improving GUI or sensing grips. Styluses slide across a glass surface making the experience not like a real paper or pen like what we are used to.

Conté explored the design space of a multimodal crayon that can exhibit mode switching techniques by utilizing the different contact points from a prism-shaped crayon [58]. Their device addresses common issues found in unimanual pen mode switching and inputs where the offhand was used for mode switching while the stylus hand was used for the primary interaction $[25,26]$. They proposed that because of the different contact points and physical features presented from a prism-shaped crayon, users can switch between different input modes by changing Conté's point of contact with the surface.

WatchPen addressed the issue of large GUI menus that disrupt drawing or that take up precious screen space [27]. They integrated an interactive screen to the end of the stylus that can receive and output information for the user. They were able to control parametric inputs such as brightness, tone, and saturation on the screen with the nondominant hand thus, not interrupting stylus workflow. I/O Brush explored adding a camera to the brush side of a stylus to read colour information from the real world to be used on digital drawing 
applications [47]. They propose this feature to be helpful for digitally recreation of realistic artwork by obtaining accurate colour values.

\subsection{Pen Grip Techniques}

We explored the literature of different pen gripping techniques as it played an important contribution to designing the ergonomics and physical features pertaining the HyperBrush prototype. Researchers have studied how stylus users can change their grip depending on the context of use such as writing, drawing, menu selection tasks or mode switching $[3,12$, $25,26,40,53]$.

Song et al. ran a pilot investigation on how their participants used a digital pen within their work environment and Hinckley et al. observed natural grip behaviours users go through while writing and web browsing $[25,53]$. Both studies defined a taxonomy of grip styles such as the tripod, sketch, tuck, or palm grips. The tripod grip is where three fingers grasp the barrel of the pen (often the index, middle, and thumb), mainly used during writing, drawing, or precision tasks $[25,53]$. The Sketch grip is where the user held the pen within the curl of the fingers, mainly used for drawing long and consistent strokes [53]. Lastly, the tuck or palm grip is when the pen is tucked away in the palm of the hand, freeing up the fingers to interact with a touch screen $[25,53]$. They concluded that the user's grip is often driven by users' comfort, fatigue or current function such as interacting with a barrel button [25]. 
Cami et al. proposed various pen postures during writing that can be configured to control short-cut keys, actions, and widgets [12]. Their input space included an assortment of postures composed of combining poses of the palm, grip, and non-grip fingers. They evaluated the usability of their postures in a realistic setting and found them to be valuable for mode switching between the pen, eraser, and highlighter tools and less desired for menu selection tools.

Matulic et al. sought to better understand pen grip postures during mode switching and asked participants what variations of pen grip they use [40]. Their results showed that most people favoured using a three-finger tripod grip while writing and more unpopular grips were ones that required a consistent pressure, such as gripping the pen firmly. They suggested that mature grips (tripod and drawing grips) are best used for maintained modes such as writing or drawing and postures that require pressure, finger extensions, or tilt, would be best configured for mode switching or menu based tools (tuck, finger extensions, or barrel presses).

From this survey, we found that the tripod grip was the most commonly used grip for writing and drawing tasks as it posed to be the most comfortable and versatile grip. We wanted design the physical features of our stylus to encourage a tripod like grip. Our first study required a higher grip relative to the barrel to minimize the screen occlusion produced from the hand. Therefore, we designed our HyperBrush for study 1 to encourage a tripod like grip with a pseudo-button placed above the flexible barrel to encourage the placement of the index finger. 


\subsection{Pen-Based Menu Designs}

Most applications offer their tools at the edges of the display, therefore having to travel across the display to interact with ultimately, disrupting the user's workflow and accumulating time and errors $[32,33]$. In this section, we look at research that has explored marking menu techniques that aim to enhance efficiency and find ways to overcome the shortcoming of lack of space.

A Marking Menu is a circular contextual menu centred around the cursor that enables users to perform selection tasks by using pen gestures and strokes, in place [32]. Stylus's benefit from marking menus speed, efficiency, and easy learning curve for novices to become experts [32, 33]. Grossman et al. found that their pen gesture-based technique Hover Widgets performed faster than traditional command activation techniques [20]. The disadvantage is that accuracy is restricted by the number of items within the marking menu, where performance starts to decrease with more than 8 items within a level $[20,28,56$, 63].

Tilt menu [57] presents a technique that utilizes tilt interaction to select items in a radial menu. For instance, the user would tilt the pen in the direction of the item they intend to select. They use three menu sizes with items of 4,8 , and 12 and evaluate the completion time and error rates from participants. Their results concluded that with more items in a radial pie menu will lead to higher error rates and completion times. They also found that ill-located items that are occluded by the user's hand during task selection may result in higher error rates. 
Ahlstrom et al. proposed their square menu design and conducted a comparison study to traditional and radial menu designs [1]. They concluded that their square menu design can outperform or perform similarly to the other traditional menus. They found that although the completion times are the slowest out of the three menus and that it could likely outperform the other menus when more trials were presented or with experienced users.

Hinckley et al. researched to improve marking menus by increasing the number of submenus available [63]. Menu breadth is defined as the number of items around the menu and menu depth is the number of submenus available. For instance, if a menu consists of a breadth of 8 and a depth of 2 , therefore a total number of 64 items are available to select. They also evaluated how many items can be in each hierarchical level before items become too slow to select or prone to errors. They concluded that when there are more than eight items, performance starts to decrease at each level of depth. The number anything greater than eight items show decrease in efficiency and accuracy. A reasonable accuracy rate should be greater than $90 \%$.

PushMenu, also determined that it is difficult for users to select items when depth is greater than 8 [26]. Their menu technique utilized pressure input to switch between different levels of eight items. Their experiment tested five levels of pressure that was directly mapped to five menu levels with a consistent breadth of eight items. They distinguish the selected level by enlarging the ring and colouring white while the other two levels remained gray and decrease their size. They inherently used the pens X-Y position for item selection 
within that menu. From their results, they state that a 2-level Push Menu with 16 items performs similarly to the traditional 8-item Marking Menu. In conclusion, they achieved increasing the number of items available without compromising time or accuracy.

Flower Menus are another variation of Marking Menus, which supports straight and curved gestures to encourages large number of items within a menu [6]. They ran a pilot study with right-handed participants and instructed them to perform Flower like gestures in order to accumulate a database of effective gestures. Their results demonstrated that straight lines are the fastest to perform and the more complicated the curve, the slower the performance is. With this information, they were able to assign where items are located based on their frequency of use. What makes their Flower Menu design more effective than Polygon Menus is the ability to group like items together. Their design is modular so that designers can create meaningful item groups in either breath or depth for organization. Their design is split into four orientations (north, south, east, and west) that each contain up to seven items each distinguished with a specific gesture. They designed seven gestures that are unique for each of the four rotations.

Hover Widgets [20] use small pen gestures or quick movements to activate a localized widget interface. In their experiment, they found that their method was faster than traditional command activation techniques. During stylus use, their users had to move across a screen to interact with menus and widgets which can lead to error-prone situations and add unnecessary time translating across the screen thus, dividing users' attention which can lead to more error-prone situations. Users must activate the widget by forming an " $\mathrm{L}$ 
shape" gesture with the pen. This is because the hover gestures and susceptible for accidental input and needs to be controlled with an activation toggle.

We reviewed different pen-based menu interfaces and implemented a radial menu design to be used as the testing application for our first study. We structured our study protocol and application around previously explored work such as evaluating the menu sizes of 4 , 8, and 12 items previously done by Tian et. al.

\subsection{Deformable User Interfaces}

Standard input tools such as the mouse and keyboard require a combination of indirect operations for complicated tasks in 2D or 3D shape manipulation [43]. Early research from Murakami et al. proposed using real deformable objects as input tools to provide users with more tactile and an intuitive feeling [43]. Their graphic interface modelled a virtual representation of their deformable cubic object. When the object was deformed: twisted, pressed, lifted, or squeezed, the virtual object would display the deformation within their interface. There is now a growing field of research in HCI dedicated to exploring physical interfaces that can deform by the user to be used as a form of input [37]. This is known as deformable (DUI) or bendable user interfaces which try to achieve a more intuitive and tactile interaction with physical objects $[38,43]$. DUIs are defined as devices that have components of soft or malleable material that require some physical exertion from the user and used as an input technique [9]. DUIs also made its statement in gaming [16, 51], accessibility [10, 15], electronic reading [59], and mobile handheld devices [19, 34, 39, 54] that replace or augment rigid input [9]. Research has also made it important to consider 
how the physical properties of stiffness [29, 30], material [38], and size $[36,44]$ of the device can influence user's preference and performance. This section discusses research that focused on evaluating the effects of materials properties on the user's performance and preferences while using a deformable device.

\subsubsection{Measuring Flexibility}

When researching flexible devices, it is also important to discuss how flexible something should be which can vary due to the type of material, shape, and density [30, 35, 37]. Kildal et al. explored the influence of flexural stiffness on users' performance [30]. Their experiment evaluated how precisely users can apply and maintain target levels of force by comparing the applied users force and target force. They find that the devices stiffness level did not affect the task performance, however, user comfort and preference favoured softer materials than stiffer materials. Kildal et al. also shows that increasing stiffness negatively influences performance and preference [29]. Their device interaction has the user bend the ridged edges of the device either upwards or downwards resulting in a flexed position. To classify the stiffness of the device, they looked at the relationship between the force being applied $(M)$ and amount of deformation in degrees $(\alpha)$. This is known as the rotational stiffness $(k)$ and a variation of Hooke's Law (1).

Lee et al. studied different flexible properties from different materials such as the paper, plastic, and cloth when studying how users manipulate a deformable display [35]. They found that paper and cloth-like material presented more input capabilities as compared to the plastic sheet. They found that paper and cloth material can be bent, twisted, crumpled, 
teared, rolled, and also stretched for the cloth material. While the plastic sheet can only be bent and twisted as forms of input, plastic is the closest material to current flexible displays.

Lo et. al designs a deformable user interface that mimics the material properties of a commercialized flexible device [37]. Their prototype Bendy, is made up of compositions of plastic and silicone material. They experiment with the shore hardness level (resistance of the material to permanent indentation) to create a prototype that can withstand repeated strain and bending without any permanent damage. They fabricated their prototype using three different flexibility levels by using different silicone resins rated at shore hardness levels of $70 \mathrm{~A}, 60 \mathrm{~A}$, and $30 \mathrm{~A}$. They found that the hardness levels greater than $30 \mathrm{~A}$ were significantly more difficult to perform bend gestures with. Our initial Thermoplastic Urethane (TPU) prototypes had a shore hardness level of 95A while our Elastic Polymer prototypes had a level of 50A being much softer material.

Shahmiri and Dietz developed ShArc, a bend or shape sensor capable of accurately measuring multiple bends along its length [49]. They achieve this by measuring the relative shift between two coherent electrodes from the bottom and top layers. They used a relatively simple technique that is also used in digital calipers that can measure change in position. The benefit of this sensor is that it allows for a robust sensor that can accurately reproduce curves with multiple bends while not being affected by angular errors which sum up each joint measured like jointed systems. 


\subsection{Previous Designs}

The concept of a flexible stylus interface has been introduced by Fellion et al. [18]. In their study, they evaluate the accuracy of bend as a variable input changing the brushstroke width of a digital paint brush. For a flexible pen, they propose three grip styles, tool grip, menu grip, and in air grip, each used for a different context during stylus use. Our first experiment expands and focus on their menu grip design, which enhances the range of motion from the wrist. This is achieved by adjusting the user's grip at a higher location along the barrel of the stylus allowing rotational bend input of $\left[0^{\circ}, 360^{\circ}\right]$. In our study, we use this grip mode to enable full range of motion for our radial pie menu selection tasks in Experiment 1.

FlexStylus's sensor was constructed from four fibre-optic flex sensing cables to measure bend input from four directions thus creating two new input modalities: the amount of flexion and direction of flexion [18]. For our HyperBrush, we used a commercial capacitive bend sensor that provided more reliability, robustness, and precision. Compared to FlexStylus's physical design, we implemented a modular design purposed to interchange flexible components which allowed us to evaluate the effects of flexural stiffness.

The original FlexStylus was also designed with a fixed flexibility strength that was not suited for our study's goals. Our goal for our studies was to evaluate performance and preference for different flexible styluses. In order to achieve this, we had to be able to prototype multiple styluses that vary in stiffness. We began with researching on which fabrication methods and materials should we utilize. 


\section{Apparatus Construction \& Design Process}

\subsection{Introduction}

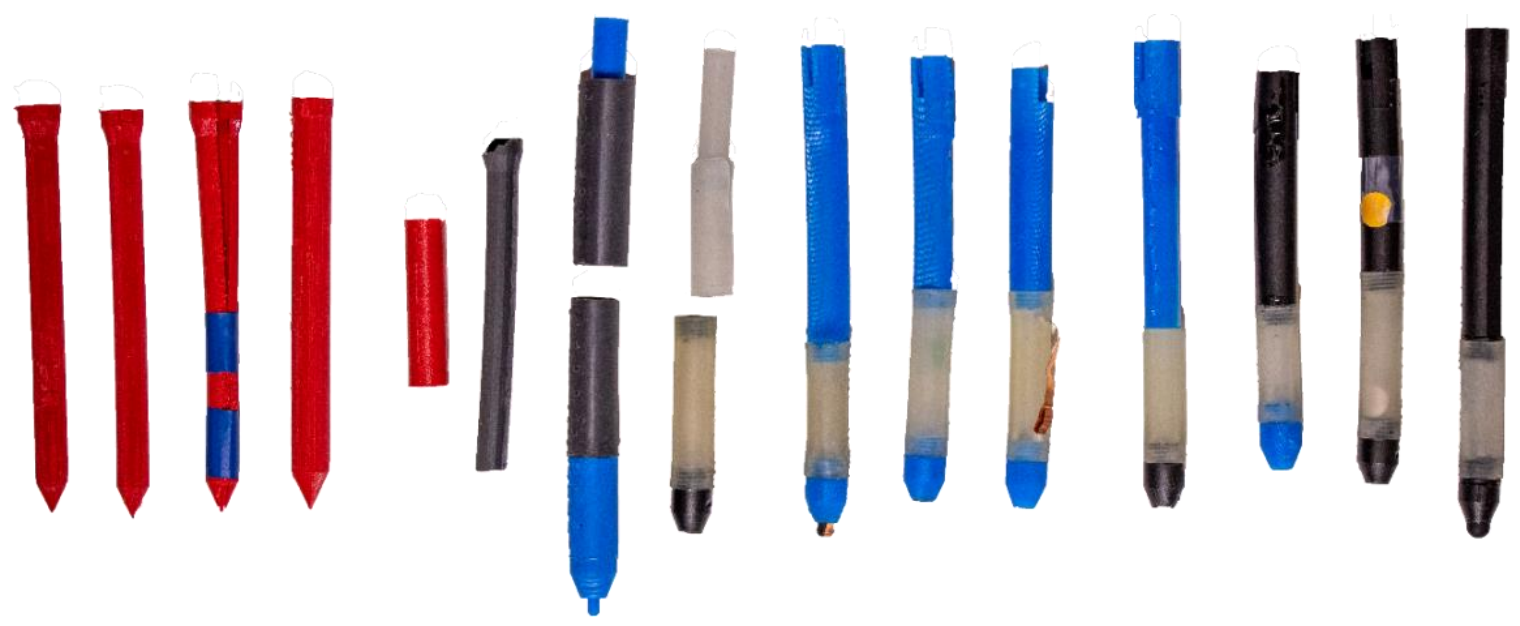

Figure 5. Collage of iterations of prototypes made from a uniform single material (red thermoplastic urethane) to a multi-material design (blue, black, and transparent photopolymer resin).

We designed HyperBrush, a flexible stylus that features interchangeable components that vary in flexural stiffness. We decided to have a modular design and separated our stylus into three main components that attach the upper barrel, flexible barrel, and tip. Our pen design enabled swapping of the flexible section, interchangeable amongst different stiffnesses. In this section, we discuss our design process from sketching to $3 \mathrm{D}$ printing, the design of physical components, and compare our sensing technique to previous designs.

\subsection{D Modelling}

We started with initial sketches that were drawn on paper and then moved towards a professional 3D modelling software, Autodesk Fusion 360. We choose this software because of its convenient 3D modelling tools such as Bevel, Extrude, Curve, and Hollow. 
These tools eased our modelling process of constructing complex curvatures and polygons with precision.

\subsection{Fabrication Methods}

In this section we explain what fabrications methods were used to materialize our HyperBrush prototype. We decided to $3 \mathrm{D}$ print our device as it allowed us to rapidly prototype and iterate through different designs.

\subsubsection{Fused Deposition Modelling 3D Printing}

We used the Ultimaker 3 Extended, a commercial Fused Deposition Modelling 3D printer (FDM) that allowed us to print at an Extra Fine layer height $(0.01 \mathrm{~mm})$. We printed our HyperBrush out of a flexible plastic material, Thermoplastic Urethane (TPU), which presented qualities similar to rubber or plastic. We modified the amount of flexibility of our prototype by changing the material density also known as the infill setting on the Cura 3D Printing Software. Cura enabled us to control printer settings such as the, material type, infill, print temperature. We used the Cura software to calculate the operations needed for the Ultimaker 3D printer to fabricate our prototypes. We attempted to have the lowest infill setting of $0 \%$ to achieve the most flexible prototype that the Ultimaker can print.

We attempted printing at $0 \%$ infill from our Ultimaker, we could not achieve the desired flexibility as it was too stiff to bend comfortably. We experimented with the positioning of the stylus on the print bed to be placed both horizontally and vertically when printing. We noticed that when printing the pen in a vertical position, print layers were perpendicular to 
the force being applied during bending. We suspected this was due to the fact that the areas between each print layer are considerably weaker.
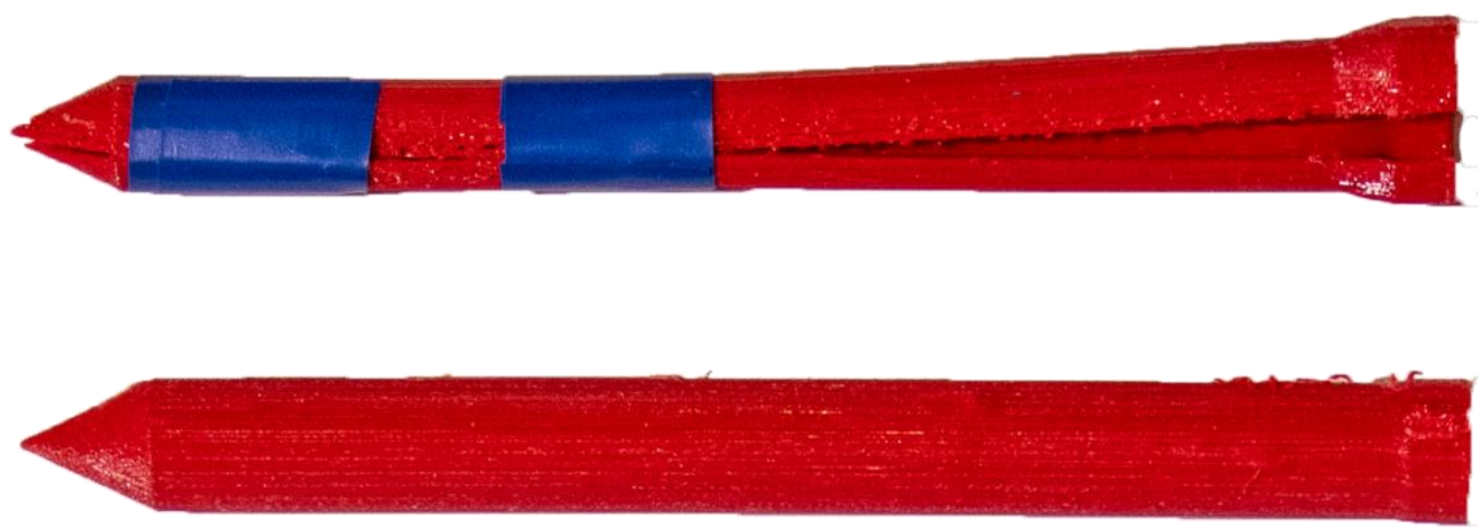

Figure 6. Early design of a TPU flexible stylus prototype that was printed horizontally with PVA water soluble support material. (Top) Prototype split into two held together with blue electrical tape. (Bottom) Thicker prototype that did not split but difficult to bend.

Our next trial was to print our prototype horizontally on the print bed which resulted in slower print times and the additional requirement of water-soluble support material, Polyvinyl Alcohol (PVA) (Figure 6). The support material was used to support upright overhangs to address the curvature design of our pen and then removed after by dissolving in water. We noticed that the prototype was able to bend without snapping in half. However print layers were now parallel along the pens barrel being more susceptible to sheering and peeling along the length of the pen.

While FDM printing allowed us to rapidly prototype designs to test general sizing and ergonomics, it did not give us the precision, quality, and robustness needed. Therefore, we moved towards other methods of fabrication that produced higher quality and robustness for our HyperBrush. 


\subsubsection{Stereolithography 3D Printing}

We began researching into Stereolithography (SLA) printing, which is well known for greater precision and quality in comparison to FDM printing, however, print times tend to be much longer than FDM printing. We used the Form Labs 2 by FormLabs Inc. SLA printer, along with Elastic Photopolymer resin with a tensile strength of 3.23Mpa and a shore hardness of 60A. Previously were able to control the infill amount for FDM printing to obtain different flexural stiffnesses. The disadvantage for SLA printing is that we have no control over the amount of infill material because of its liquid resin properties. Therefore, we used a different approach by modifying the thickness of the flexible component by changing the diameter of the inner wall to vary the flexural stiffness Figure 9. We discuss the flexural stiffness properties and measurements in detail within section

\subsection{1.}

\subsection{Prototype Design}
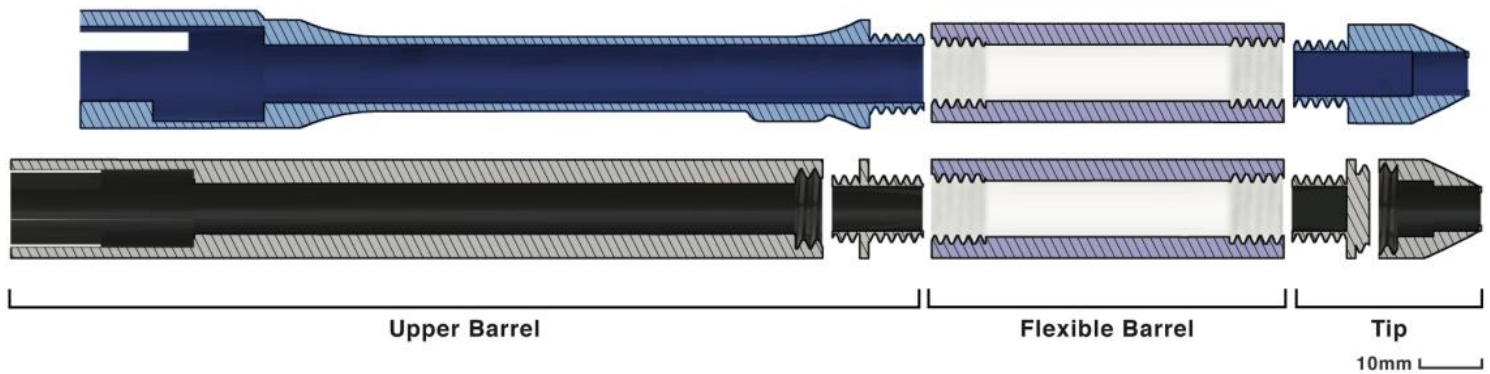

Figure 7. To scale cross-section models of both experiments stylus designs. Experiment 1 (Top), with a concave upper barrel and pseudo-button that suggests orientation of the users' grip. Experiment 2 HyperBrush (Bottom) features additional inner screws that improve durability and modularity. 

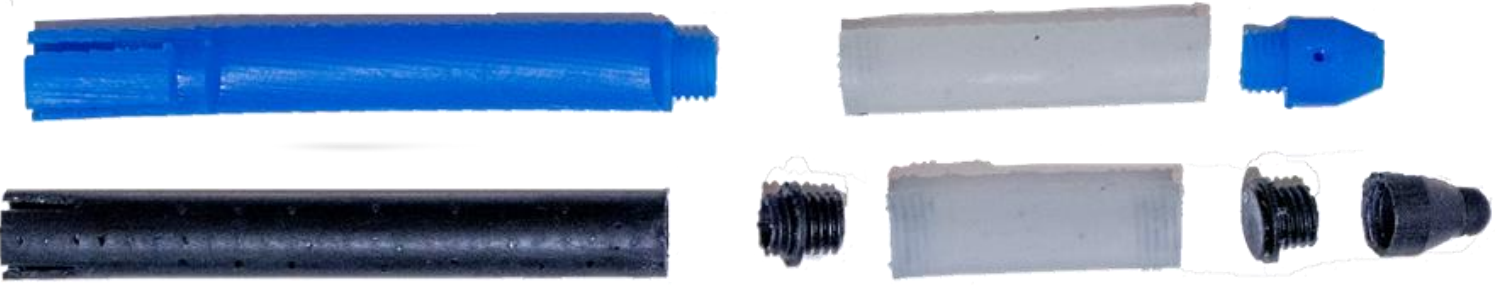

Figure 8. Top view of both final design's styluses separated apart with screw design. Early prototypes of HyperBrush were designed with a uniform flexible structure. For our purposes we only required a specific location on the barrel of the pen to be flexible, similarly to FlexStylus [18]. Therefore, we moved towards a hybrid design of both flexible and non-flexible components (Figure 8). We split the pen into three sections: pen tip, flexible barrel, and upper barrel. We decided to have the flexible barrel at a low position because for our menu interaction the user's grip shown best performance when placed above the flexible segment. If the user's grip were over the flexible barrel, it would restrict the total range of movement that the user can bend the device. If we made the ideal gripping location too high, the user's wrist would be positioned at such an extreme angle that it would become uncomfortable after a short period of time. From our pilot study, we had qualitative feedback that supports our grip design decisions.

\subsubsection{Construction of Flexible Barrel}

To maintain a uniform outside diameter, we varied the stiffness by manipulating the inner wall thickness of the barrel (Figure 9). We choose not to change the material in order to vary flexibility because we did not want to compromise the physical texture properties between each prototype. We aimed at having each HyperBrush flexibility type indistinguishable by visuals and touch. Our first study we studied four HyperBrush's at soft, medium soft, medium hard, and hard flexibility while our second study had only three 
soft, medium, and hard flexibility. We explain our rationale further in our discussion section from study one.

We classified each HyperBrush's flexibility by calculating the flexural stiffness as a ratio of amount of force applied per unit of deflection. We equated the flexural stiffness for a cantilever beam modified for a hollow cylinder to classify each HyperBrush's flexibility [48].
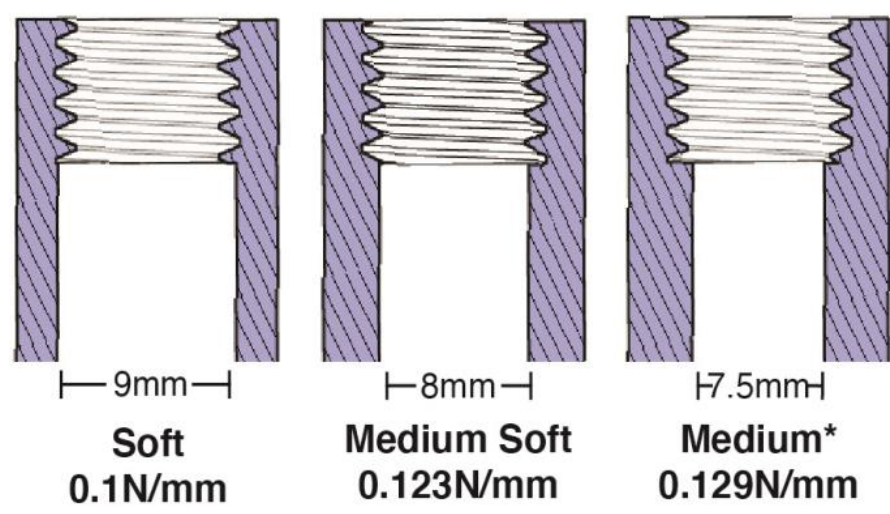

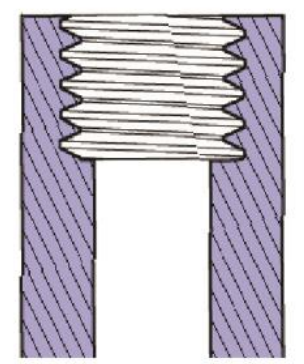

$1-7 \mathrm{~mm}-1$

Medium Hard

$0.134 \mathrm{~N} / \mathrm{mm}$

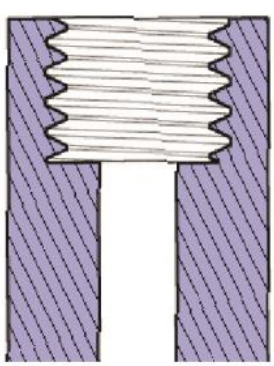

$\mid 6 \mathrm{mml}$

Hard

$0.139 \mathrm{~N} / \mathrm{mm}$

Figure 9. Front cross section view of the five 3D printed flexible barrels with flexural stiffness measurement not including the bend sensor inside. The medium* flexibility was used for the second study.

\subsubsection{Flexural Stiffness}

We classified each flexibility as a ratio of amount of force applied per unit of deflection known as the flexural stiffness. We describe this relationship $k$, as the ratio of the torque applied $M$, and the angular displacement produced $\alpha$. This is also known as the rotational equivalent of Hooke's Law [30], (1). 


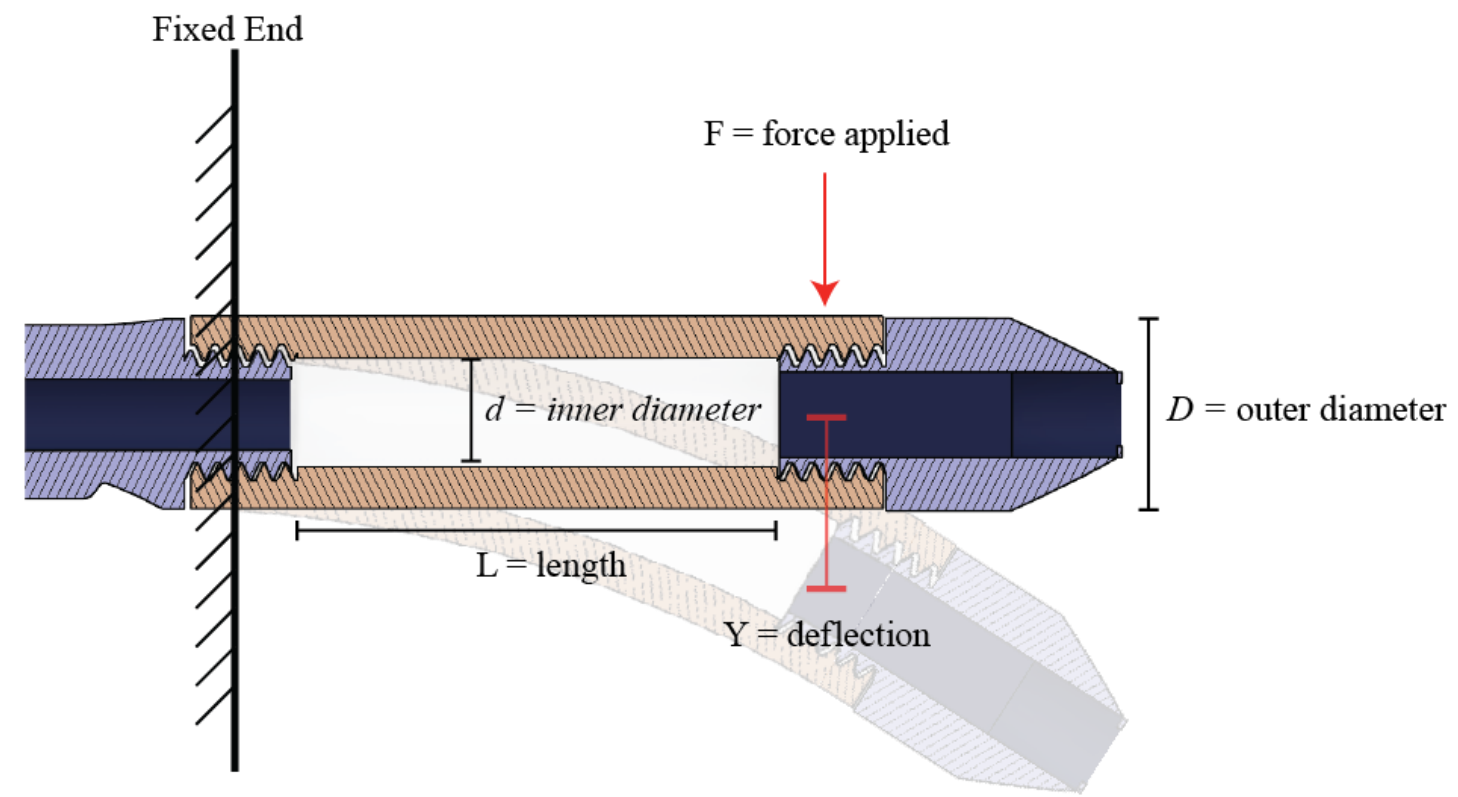

Figure 10. Experimental setup for a beam deflection test. HyperBrush being suspended at the fixed end and a load force applied at the other end of the flexible barrel (orange).

We calculated the flexural stiffness similarly to how a hollowed cylinders stiffness is calculated on a cantilever beam test in engineering design $[48,55]$. Figure 10 shows a cantilever test where our HyperBrush is suspended horizontally with a downward load force, $F$, is applied resulting in a deflection, $Y$, along the flexible barrel $[48,55]$. Flexural stiffness is then calculated as a ratio of, $k=\frac{F}{Y}$. We found this experimental setup appropriate as it simulated the force of a user bending the stylus during interaction.

The cantilever test required precise measurement tools, various weights to be hung from the device, and manual measurements to be recorded. However, when given the Area Moment of Inertia, I, (2) and the Youngs Modulus, E, of our device, we can equate the flexural stiffness (3). Young's Modulus is a constant measurement defined as the mechanical property of stiffness of a given material [62]. Our elastic photopolymer resign 
was given a Young's Modulus of, $E=3.23 \mathrm{MPa}$ based on BendLabs material data sheet. Our final calculations are presented on Figure 9.

$$
\begin{array}{lc}
\text { Hooke's Law } & k=\frac{M}{\alpha} \\
\text { Area Moment of Inertia } & I=\frac{\pi \cdot\left(D^{4}-d^{4}\right)}{64} \\
\text { Flexural Stiffness } & k=\frac{3 \cdot E \cdot I}{\mathrm{~L}^{3}}
\end{array}
$$

The flexible segment of the pen was riveted on both sides so that the pen tip can screw on one side while the rest of the pen shaft can screw on the opposing side. We implemented this design because we wanted to interchange flexibility and exchange parts that have been damaged during use. However, during testing the screw design would not hold the parts together when flexing at extreme angles. To prevent tearing of parts, we had to apply additional adhesive inside the screw segments. We decided to have duplicate prints of pens ready to go if one breaks during testing for further precaution. 


\subsubsection{Construction of Top Barrel}
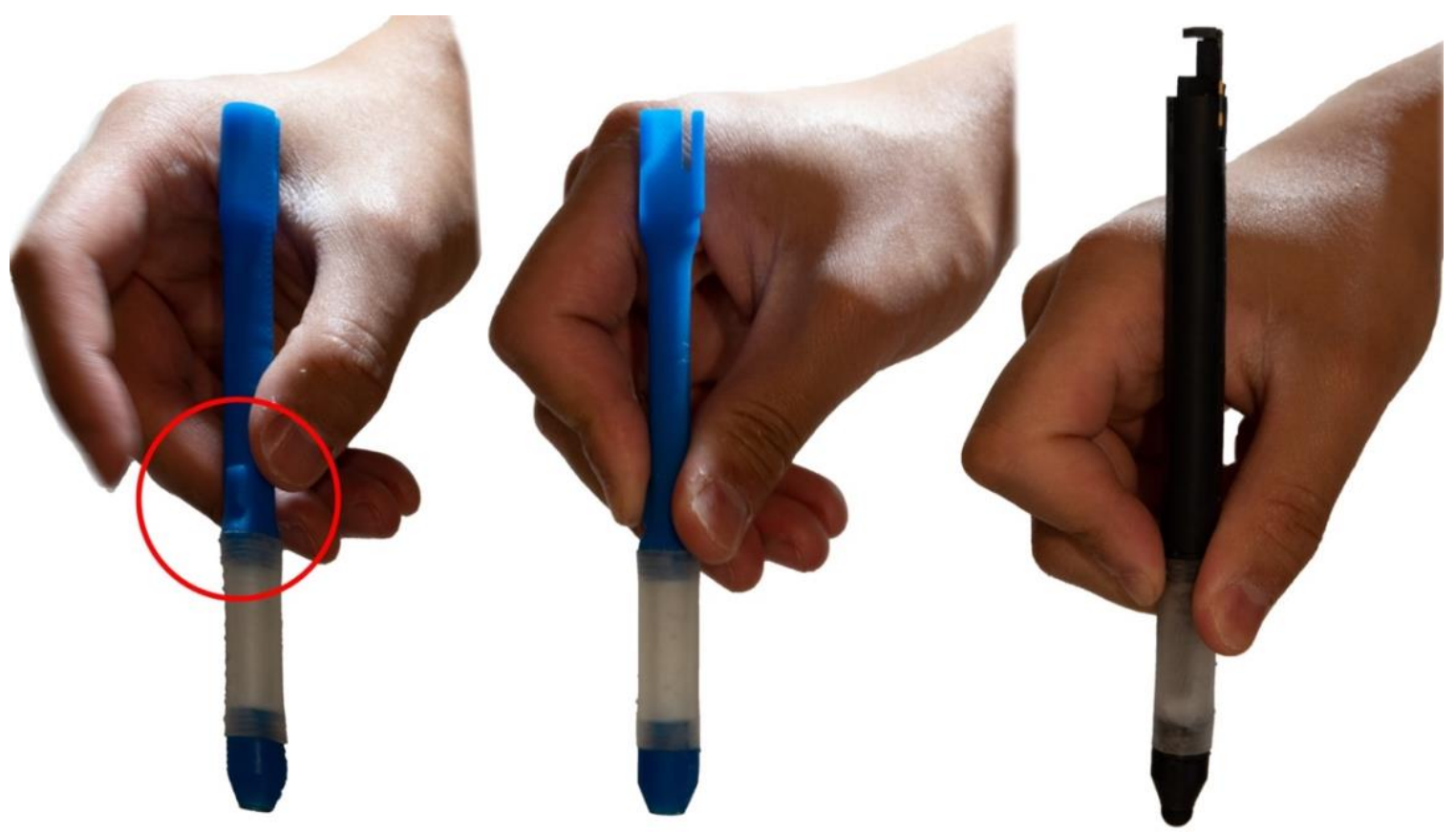

Figure 11. HyperBrush designed for menu selection experiment with pseudo-button (Blue) and the HyperBrush design for the brushstroke experiment (Black). Circled in Red is the pseudo-button.

For our radial menu selection study (Chapter 4), we wanted participants to holster HyperBrush at a high position along the pens barrel to allow full range of rotational bend motion (Figure 11). We also wanted to reduce the amount of natural or accidental rotation of the HyperBrush from the user during use. Therefore, we drew our focus towards its ergonomics, grip, and thermoplastic overall comfort. We noticed that participants naturally regrip and rotate the pen within their hand. In order to read our sensor data properly, our software required the device to be oriented in a specific direction. While a gyroscope could account for this issue, which could calculate the rotational offset and neutralize the rotation axis we decided to change the physical design of the pen to guide the affordance of the grip. 
As an alternative, we added an extrusion on the barrel, a pseudo-button as an indication for where the users must place their index finger. We noticed that participants were able to maintain their grip better and produce fewer natural movements that resulted in accidental rotation. We also modified the curvature of the barrel so that it is more ergonomic to holster. Instead of having an equidistant ellipse, we moved towards having an oval shape to adhere naturally to the user's grip.

\subsubsection{Construction of Pen Tip}

We designed the pen tip to be made from rigid material as it is not a requirement for it to be flexible. We attached a rubberized conductive nib that was deconstructed from commercial digital styluses. We connected it to copper wire cut from a sheet that was placed along the outside of the pens barrel. This allowed us to transfer electrical capacitance from a user gripping the pen, to the pen tip, then to the surface of the tablet ultimately registering as a touch input device.

From feedback given from our first pilot test, we had to redesign where the copper wire was placed. Users were frustrated always trying to regrip the pen and maintaining their finger on top of the copper wire in order for the stylus to work. Our solution was to move the copper wire within the same cavity as the sensor. Fortunately, the sensor worked off of capacitance therefore the copper wire read the capacitance off of the sensor instead of the user's hand. 
To connect the pen tip to the flexible segment of the device, we implemented a screw design with additional adhesive glue so that the tip would not snap off during bending (Figure 8).

\subsubsection{Bend Sensor}

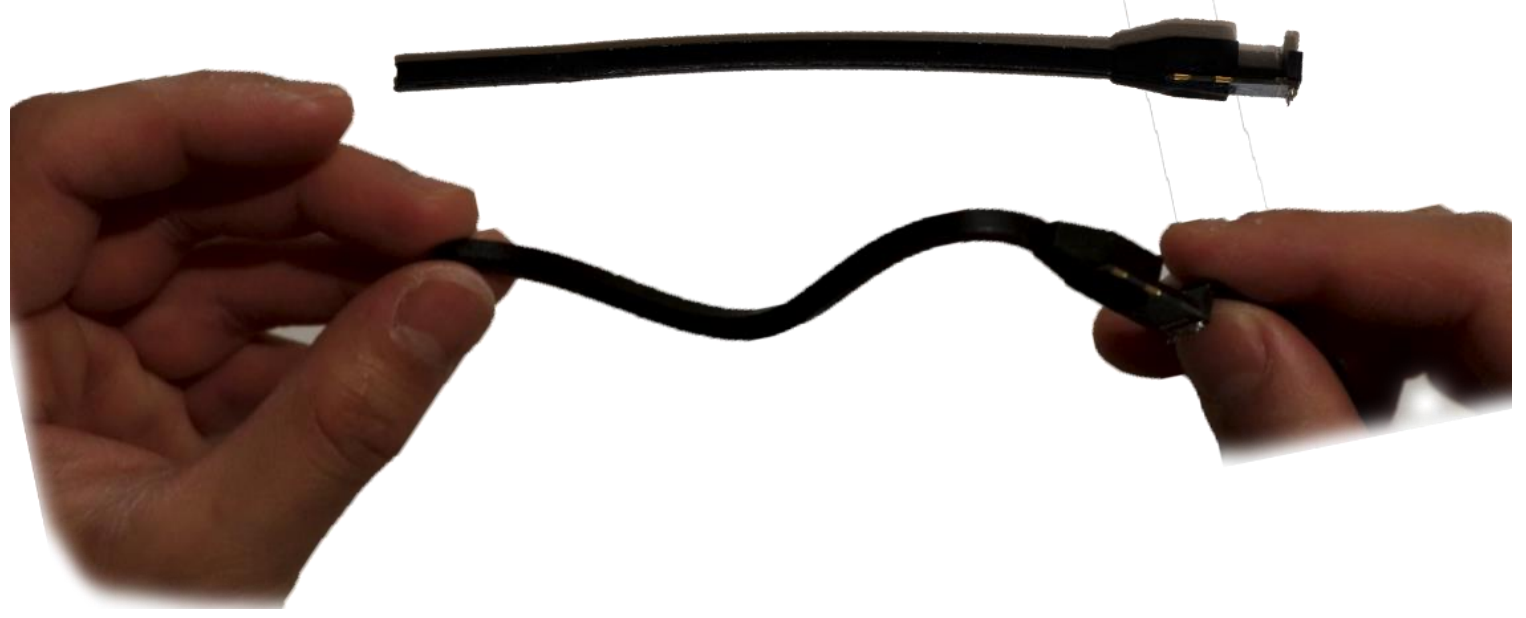

Figure 12. BendLabs Inc. two axes soft angular displacement sensor.

We used the soft angular displacement bend sensor (Figure 12) by BendLabs Inc. [7]. Their sensor is made up of two capacitive flexible sensors positioned perpendicular to one other measuring bend input from two orthogonal planes. The sensor only measures the angular displacement at the two ends of the sensor therefore any additional superimposed bends will be ignored [52]. They measure the differential capacitance between the two parallel capacitors which is used to calculate the angular displacement of the plane. This technique offers a high precision sensor that rejects most strain, noise, and temperature factors that can disrupt output accuracy. As per the diagram below, we obtained two measurement angles produced from the $\mathrm{X}$, and $\mathrm{Y}$ planes. We coupled the angle measurements to the $\mathrm{X}$ and $\mathrm{Y}$ position of our bend cursor for the bend menu study. Figure 13 shows that when the 
sensor is bent, it produces a corresponding $\mathrm{X}(\varphi=[180,180])$ and $\mathrm{Y}(\theta=[180,-180])$ where the unbent state is at $(0,0)$ degrees.
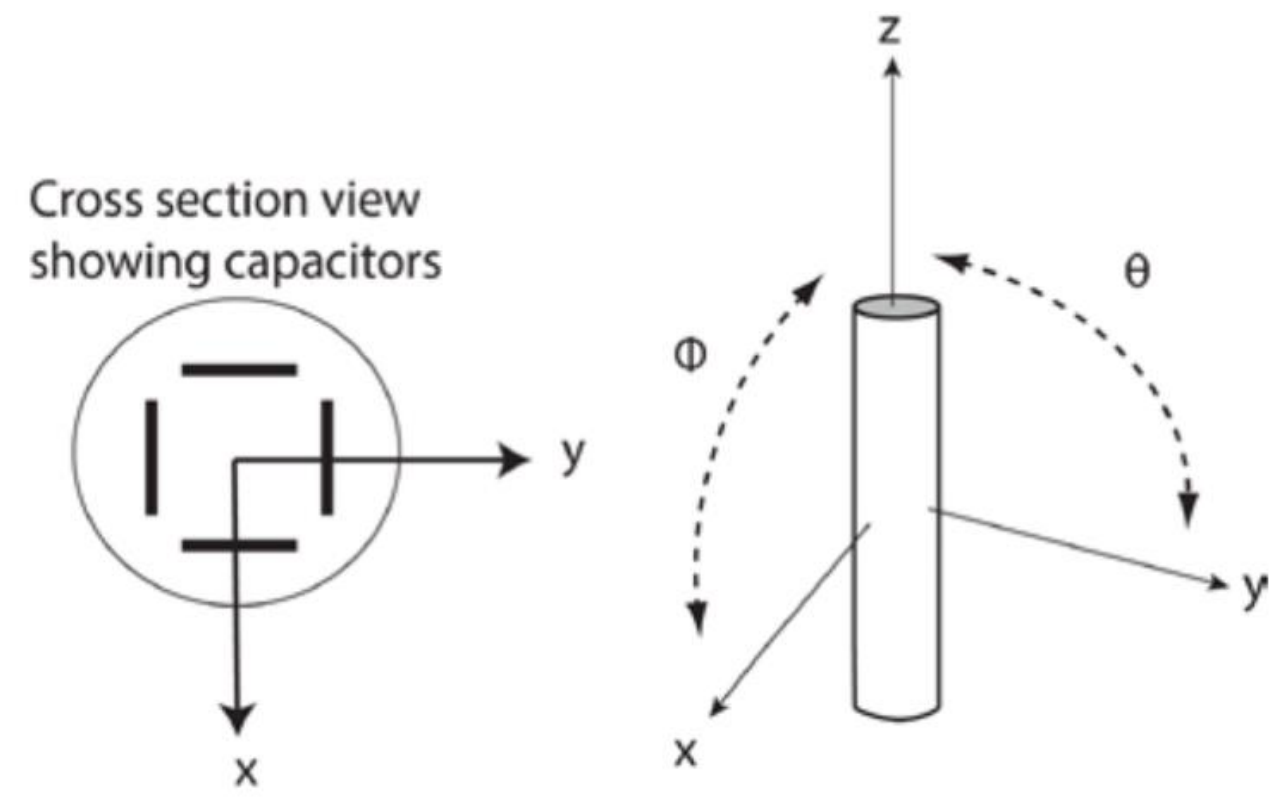

\section{Two Axis Measurement}

Figure 13. - Left. Top cross section view showing the paired capacitors for the $X$ and Y planes. Right. Perspective diagram of the barrel and its bending axis [52].

BendLabs design utilized parallel compliant capacitors that run along the length of the sensor in both $\mathrm{X}$ and $\mathrm{Y}$ directions (Figure 13). The sensor is constructed from four silicone capacitors that run along each side of a flexible rectangular material. This is so that they can measure the difference in capacitance between two capacitor sensors then they can calculate the angular displacement for that plane.

ShArc, developed by Shahmiri and Dietz, developed a bend sensor capable of accurately measuring multiple bends along its length producing a shape or curve [49]. They achieve this by measuring the relative shift between two coherent electrodes from the bottom and top layers. They used a relatively simple technique that is also used in digital calipers that 
can measure change in position. The benefit of this sensor is that it allows for a robust sensor that can accurately reproduce curves with multiple bends while not being affected by angular errors which sum up each joint measured like jointed systems.

\subsubsection{Microcontroller}

We used an Arduino Mega Microcontroller [4] (Figure 4) to read and send serial data information from the sensor to our touch screen tablet, the Microsoft Surface Pro (12" display at 1080 p resolution) [42]. We programmed the microcontroller to interpret the bend input from the BendLabs. This allowed our device to measure not only absolute bend, but also the azimuth angle or what we refer to as the bend rotation. 


\section{Study 1: Bend Menu Selection Technique}

\subsection{Introduction}

Our first experiment evaluated the performance and preference of flexibility within a bendable stylus. We tested four different flexural stiffnesses on a bend menu technique previously introduced by Fellion et al. [18]. In this section, we explained our testing application and discussed the outcomes from our experiment.

\subsection{Methodology}

\subsubsection{Menu Selection Design}

Fellion et al. previously introduced the technique of menu selection by using bendable stylus gestures, however further evaluation pertaining to the usability of the interaction was yet explored [18]. For our study, we decided to follow a similar study design to Tilt Menu's experiment, where we evaluated conditions of menu size and item location [56], as well as our flexural stiffness level. We designed menu selection tasks for three menu sizes each with a radius of $2.5 \mathrm{~cm}$ from the centre. Figure 14 illustrates the location of each item distributed by $90^{\circ}$ (small), $45^{\circ}$ (medium), and $35^{\circ}$ (large) where number of items increased by 4,8 , and 12 , respectively. We developed our testing application using Unity and coupled the bend input from the stylus to control the $\mathrm{X}-\mathrm{Y}$ position of the cursor. 

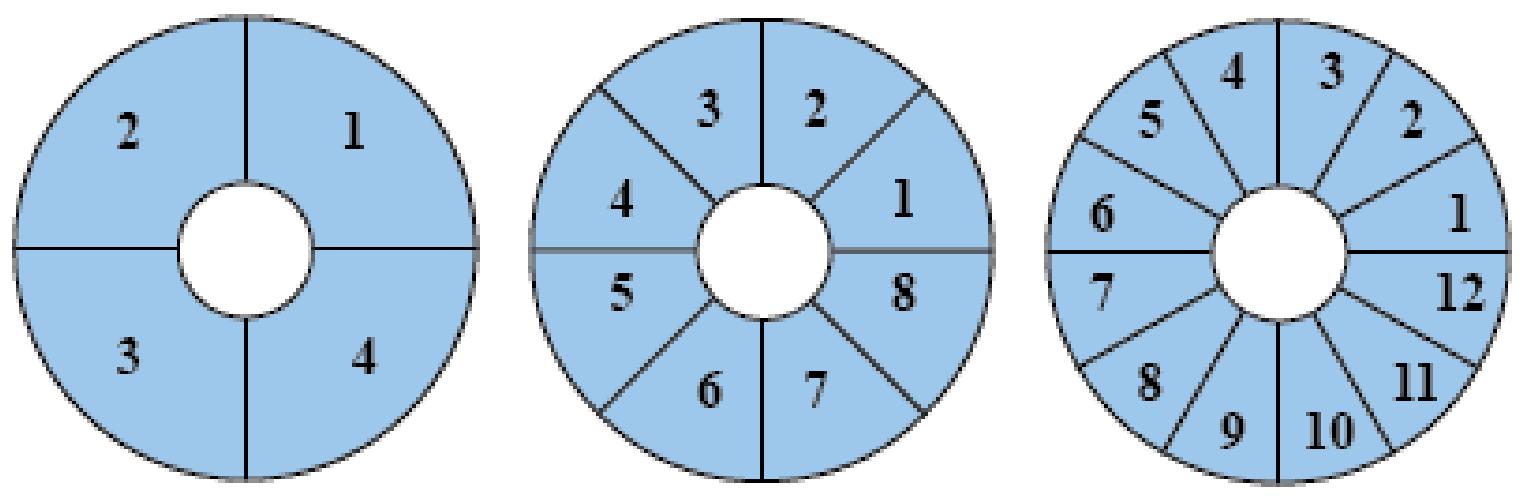

Figure 14. Radial Menu designs. Left: 4-items, Middle: 8-items, Right: 12 items.

\subsubsection{Selection Process Technique}

We applied a selection-confirmation process for our bend menu similar to Tilt Menu's other marking menu methods. We coupled the $\mathrm{X}$ and $\mathrm{Y}$ angles produced from the sensor to control the X-Y position of the bend cursor (red circle in Figure 15).

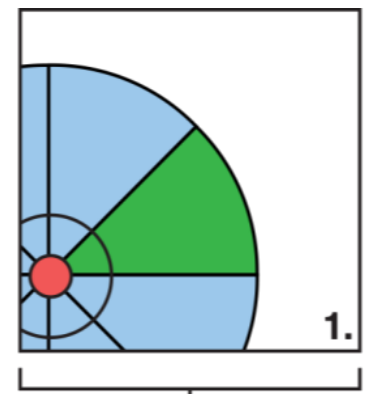

Neutral Zone

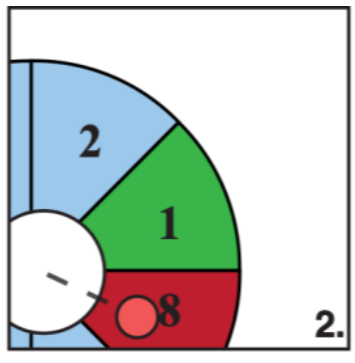

Selection Zone

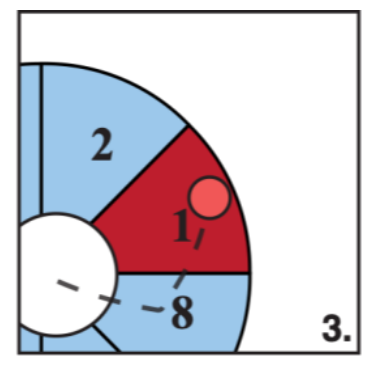

3.

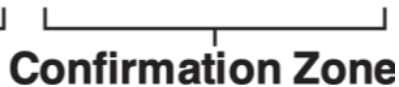

Figure 15. Menu selection process of the cursor (pink circle) to target (green item) shown in the medium size menu. 1. Cursor starting in the neutral zone. 2. Moving into selection zone (highlighted in red). 3. Navigating to target item in green. 4 . Exiting on the outside radius of the target item to confirm selection.

Search. We initialize the menu when the stylus tip comes in contact with the tablet screen.

We display the menu at which its position is attached to the X-Y position of the stylus tip

(Figure 15, 1). At this state, the user is currently searching for what item they intend to select - the neutral zone indicated in white; no current items are selected. 
Selection. Once the bend cursor is positioned in the selection zone, whichever item underneath the cursor will highlight in red indicating that it is the current item being selected (Figure 15, 2-3). The user is also able to transverse between adjacent items so that the user does not have to navigate back to the neutral zone to re-enter the selection zone of a different item.

Confirmation. To confirm the selection of an item, the user must exit the selection zone of the item by moving the cursor through the outer ring of the item (Figure 15, 4.). Once exited, the item is selected and then the menu interface disappears. We restart the process once the user touches the screen again.

\subsection{Study Protocol}

We started with a pilot study that had the additional parameter of measuring bend cursor sensitivity/speed. The study design was the same, however, evaluated a high sensitivity with a fast cursor speed and a low sensitivity with a slow cursor speed. From our questionnaire and observation, participants favoured the higher sensitivity regardless of the flexibility condition or menu size. Therefore, we proceeded to only use a high sensitivity for our experiment.

We began the experiment with a demographic's questionnaire including the participants' experience with using digital and non-digital pens. Afterwards, we briefed them with a tutorial and explanation of the experiment, which also allowed time for training and familiarity with the device. The tutorial presented a random target to select mimicking the 
actual experiment setup with no repetitions involved. We selected a flexibility type at random during this session to counterbalance learning effects.

Our experiment followed a within-subject design where participants used 4 flexible pens for each of the 3 menu sizes. We randomized the order in which participants selected each item to account for counterbalancing. Each item within each menu $(4+8+12)$ was selected 6 times for a total of 144 selections, for 4 flexible conditions (soft, medium soft, medium hard, and hard) resulting in an overall total of 576 selections.

We recorded participants selection time and error rate. We informed participants that we are more concerned with their performance rather than speed of task completion. Participants started the selection time by moving the cursor into the selection zone and then stopped when an item has been selected. We calculated participants error rate by dividing their total missed targets by their total overall targets. We recorded a missed target when the target item was not successfully selected by the user. Between flexibilities, we asked participants to fill a 5-point Likert scale questionnaire regarding the styluses perceived movement, responsiveness, and accuracy. At this time, we also encouraged participants to take a short break. We ended the experiment with a post questionnaire asking about their experience using a flexible stylus for menu selection and to rank their most to least preferred flexibility input type. 


\subsection{Hypotheses}

H1. We hypothesize that an increase in flexural stiffness will result an increase in error rate. Research has found that stiffer devices may take more physical exertion to bend, which could make selection tasks more difficult [30].

H2. We hypothesize that the hard stylus will be the least preferred flexibility, as Kildal et al. found that users preferred softer materials as they required less effort to manipulate [30].

H3. We hypothesize that the increase of the menu breadth will also increase error rate. This is supported by previous menu selection studies showing that increase in menu breadth (number of items) increases the likelihood of missing the target $[6,56,63]$.

\subsection{Participants}

Participants were recruited through email, social media, and posters. There were a total of 18 participants who participated in the study. 8 of which were male and 10 were female and 3 left-handed users. Majority of participants were University students and the average age was 23 years old. None of the participants were experienced with using a bendable device or participants from the last pilot study. Study was scheduled to be 45-60 minutes long and a compensation of $\$ 15.00$ would be provided for their time if they complete the study or not. To measure users' performances while performing selection tasks, we look at the effects of several independent variables on two dependent variables. 


\subsection{Results}

We first report the effects of repetition, menu size, flexibility, and item location on selection time and following reports the effects of the latter on error rate. Following, we discuss the preference results filled out by the participants in our survey.

\section{Dependent Variables:}

Completion Time - The total time taken from starting to confirming selection of an item. Error Rate - Represented as the percentage of the sum of failed selections over the sum of total selections.

\section{Independent Variables:}

Menu Size - the three menus with items of size 4, 8 and 12.

Device Flexibility - the four types of pens, soft, medium soft, medium hard, and hard. Repetitions - the N value between 1 to 6 , number of times a set has been completed. Item Location on Menu 4 - Items 1 through 4 locations relevant to the centre of the menu. Item Location on Menu 8 - Items 1 through 8 locations relevant to the centre of the menu. Item Location on Menu 12 - Items 1 through 12 locations relevant to the centre of the menu.

\subsubsection{Selection Time}

We conducted a multi-way ANOVA across repetition $\mathrm{x}$ flexibility $\mathrm{x}$ menu size on selection time. We found a significant effect of repetition $\left(F(5,90)=11.7, p<0.01, \eta \mathrm{p}^{2}=0.36\right)$ 
and menu size $\left(F(2,36)=124.79, p<0.01, n_{p}^{2}=0.87\right)$ however, did not find any interaction effects nor effects of flexibility on selection time.

\subsubsection{Repetition}

We proceeded with a pairwise comparison test with Bonferroni adjustments that revealed a significant difference from the $1^{\text {st }}$ to all other repetitions, $p<0.05$ for both dependent variables. We found that the $1^{\text {st }}$ repetition $(\mathrm{ST}=1.46 \mathrm{~s})$ had a significantly higher completion time compared to repetitions 2 to $6(\mathrm{ST}<1.37 \mathrm{~s})$. This suggested that learning effects started to plateau after the first repetition (Figure 16). 

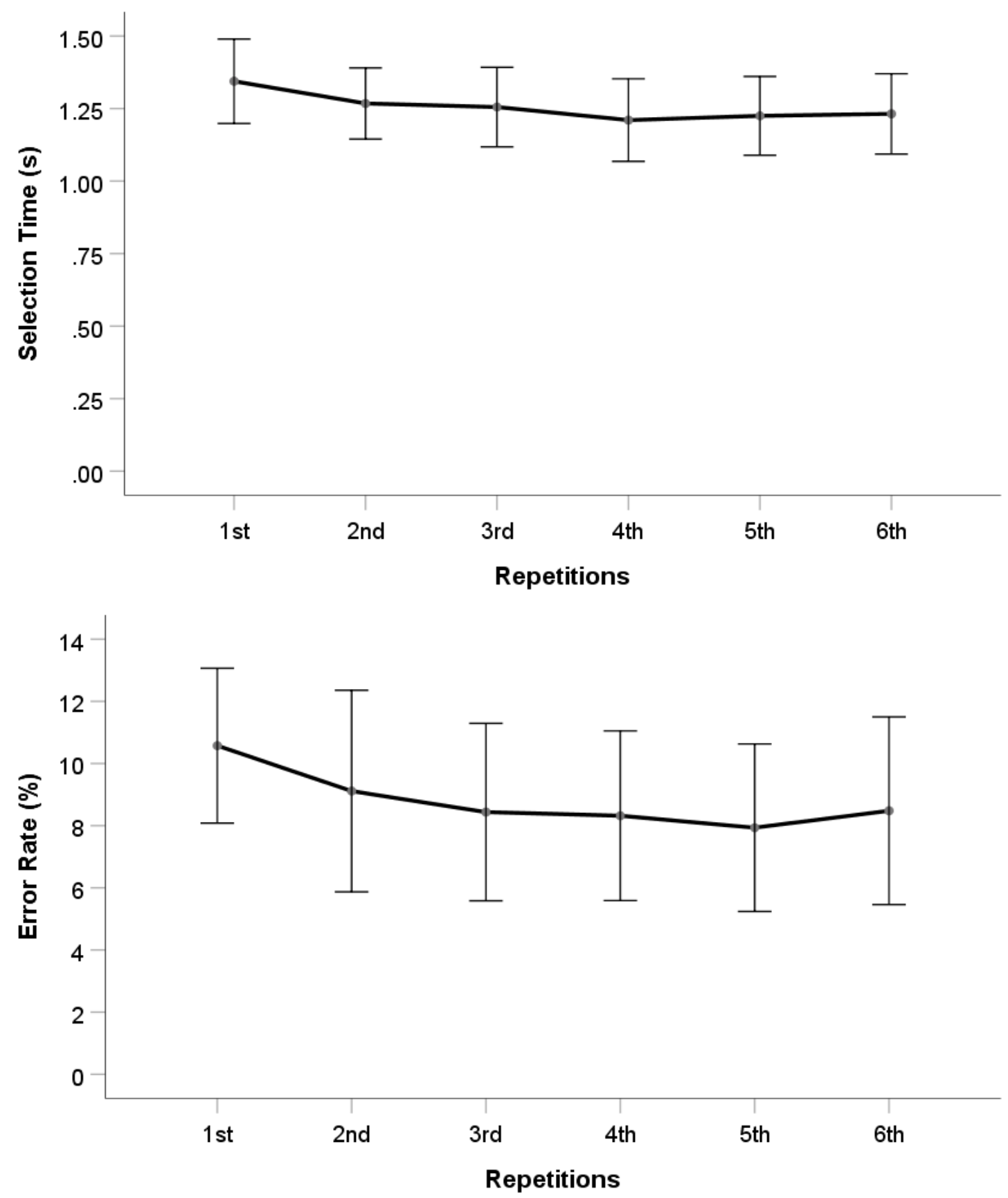

Figure 16. Average selection time and error rate for repetitions with $95 \%$ CI.

\subsubsection{Menu Size}

We found significant difference across our three menu sizes on selection time, $\left(F(2,36)=124.79, p<0.01, n_{p}^{2}=0.87\right)$. Our pairwise comparison using the Bonferroni adjustment revealed a significant difference where $p<0.01$ for each pair. This suggested 
that the increase in items within a menu also increased the overall selection time for participants. We found these results to be on par with Tilt Menu and which evaluated a ranking of menu sizes [57]. As per Figure 17, this shows that the average selection time increased when increasing from the small to large menu sizes. 

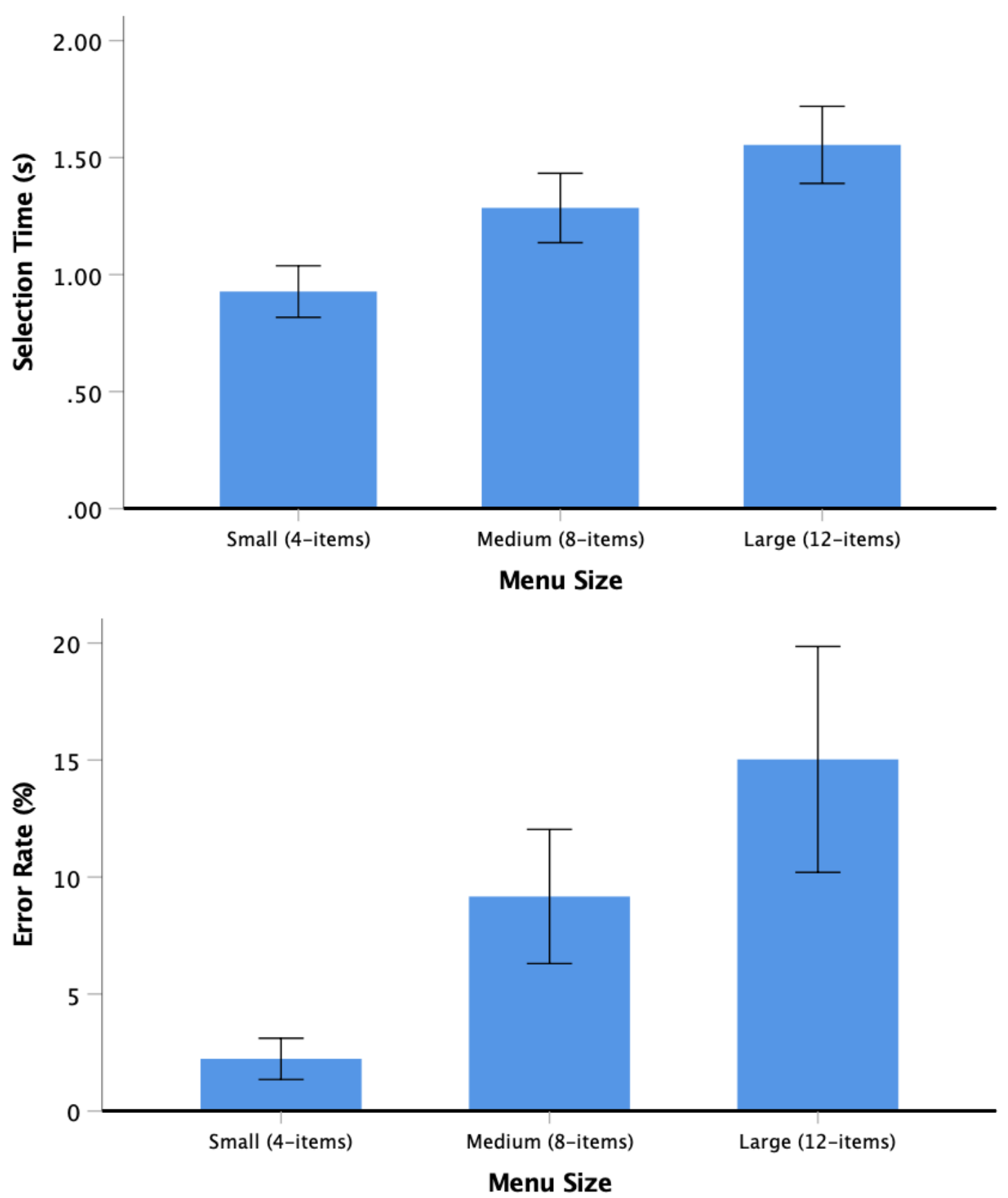

Figure 17. Average selection time (Left) and error rate (Right) with $95 \%$ CI for each menu type.

\subsubsection{Flexibility}

Our proposed hypothesis (H2) proposed that an increase in flexural stiffness will yield in a decrease user's performance. Unfortunately, we did not find any effects of flexibility type 
for both selection time and error rate. This was interesting for us, furthermore; we discussed this factor in depth alongside with our flexibility preference results.

\subsubsection{Item Location}

We were also interested in comparing each item's selection time within its respected menu. We discarded the data from our left-handed participants and evaluated the right-handed participants only $(n=15)$ to mitigate the possibility of left-hand/right-hand occlusion, similarly done by Tilt Menu [56].

Our One-Way ANOVA investigated the influence of item location on selection time and found significant differences for all three menu types: small $-F(2,27)=10.8, \mathrm{p}<0.01, \eta$ $\mathrm{p}^{2}=0.44$, medium $-F(3.3,46)=2.7, \mathrm{p}=0.05, \quad \eta \mathrm{p}^{2}=0.16$, and hard $-F(4,51)=4.5$, $\mathrm{p}<0.01, \eta \mathrm{p}^{2}=0.24$

Table 1 shows that across all three menu sizes, items located within the south-west region yielded the lowest selection times with relatively low error rates (small: \#3, medium: \#6, large: \#8, \#9). Comparatively, items located south-east were more difficult to select resulting in relatively high selection times and error rates (small: \#4, medium: \#7, \#8, large: \#11, \#12).

Table 1 - Average selection times (ST) represented in seconds and error rate (ER) in percentage per item (\#) within each menu size, grouped by location on a compass. ST coloured in red and ER in blue, where darkest shade (red or blue) indicates highest value and white indicates lowest value.

\begin{tabular}{|l|lll|lll|lll|}
\hline & \multicolumn{2}{|c|}{ Small Menu (4 items) } & \multicolumn{3}{|c|}{ Medium Menu (8 items) } & \multicolumn{3}{|c|}{ Large Menu (12 items) } \\
Location & $\#$ & ST & ER & $\#$ & ST & ER & \# & ST & ER \\
\hline North- & 1 & 0.82 & 1.33 & 1 & 1.22 & 11.33 & 1 & 1.54 & 24.02 \\
East & & & & 2 & 1.22 & 7.00 & 2 & 1.42 & 13.00
\end{tabular}




\begin{tabular}{|l|lll|lll|lll|} 
& & & & & & & 3 & 1.51 & 15.36 \\
\hline North & 2 & 0.95 & 2.33 & 3 & 1.30 & 8.67 & 4 & 1.49 & 16.11 \\
West & & & & 4 & 1.31 & 8.33 & 5 & 1.45 & 14.12 \\
& & & & & & & 6 & 1.56 & 17.07 \\
\hline South- & 3 & 0.82 & 1.00 & 5 & 1.21 & 10.33 & 7 & 1.46 & 18.18 \\
West & & & & 6 & 1.17 & 6.33 & 8 & 1.36 & 12.67 \\
& & & & & & & 9 & 1.40 & 13.12 \\
\hline \multirow{2}{*}{ South- } & 4 & 0.92 & 4.67 & 7 & 1.26 & 12.00 & 10 & 1.46 & 15.00 \\
East & & & & 8 & 1.36 & 10.97 & 11 & 1.59 & 16.67 \\
& & & & & & & 12 & 1.73 & 21.54 \\
\hline
\end{tabular}

We followed each analysis with a pairwise comparison test using Bonferroni adjustments. Within the small menu size, items $1 \& 3$ both yielded in a significantly lower completion time as opposed to items $2 \& 4(p<0.01)$. For the medium menu size, item 6's selection time was significantly lower than items $3,4,7, \& 8(p<0.05)$. We also found that items 1 , $2, \& 5$, were also significantly lower than items $3,4, \& 8$ in selection time $(p<0.05)$. Lastly, for items within the large menu we found that items $8 \& 9$ were significantly lower than items $1,6,11 \& 12(p<0.03)$. Also, item 2 was significantly lower than items 1,6 , $\& 12(p<0.03)$. From this analysis, we can conclude that items located generally in the North-East or South-West region are much faster locations to complete a selection. We further this analysis and all the latter by looking at its error rates in the next section.
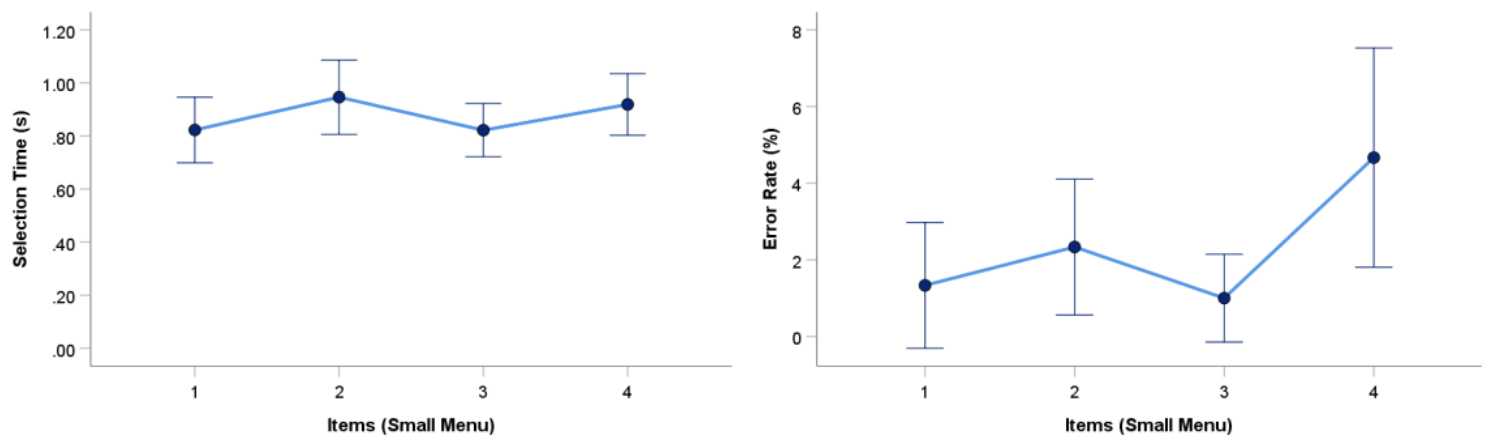

Figure 18. Selection Time and Error Rate for items within the Small Menu. 

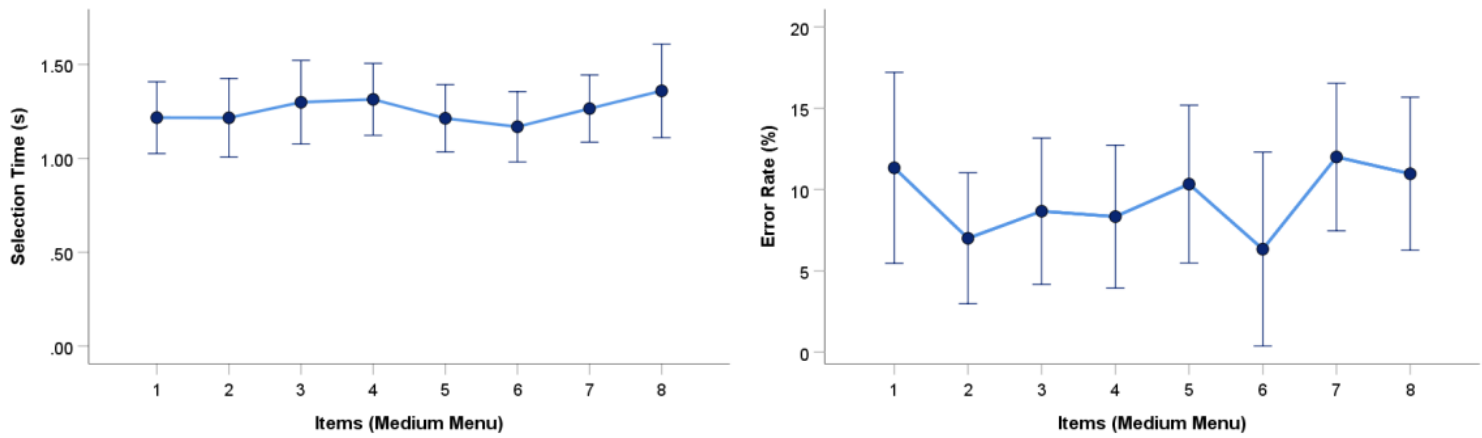

Figure 19. Selection Time and Error Rate for items within the Medium Menu.
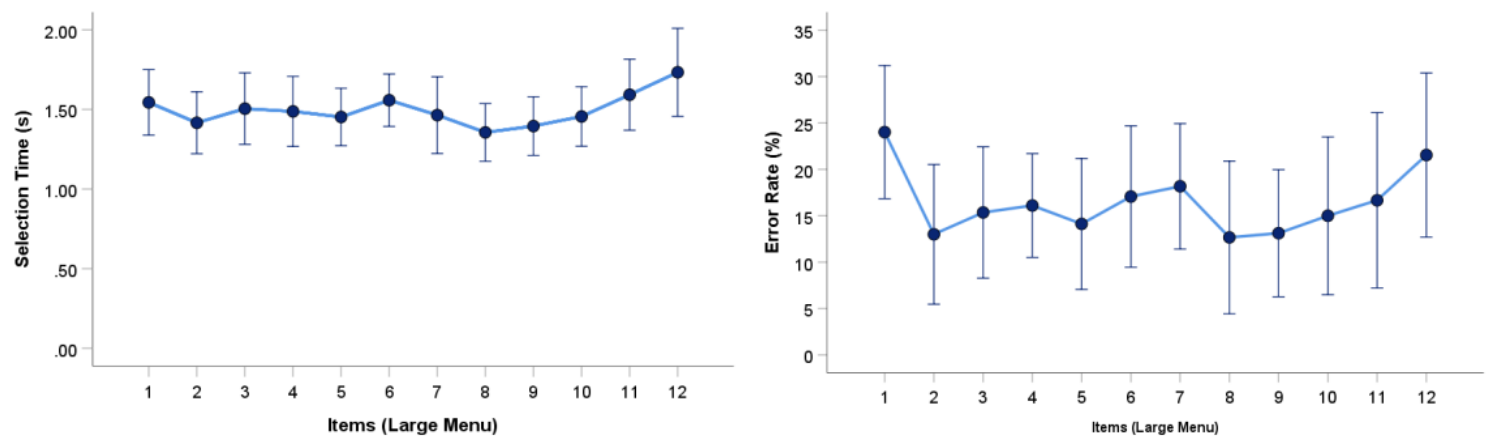

Figure 20. Selection Time and Error Rate for items within the Large Menu.

\subsubsection{Error Rate}

We conducted a multi-way ANOVA across repetition $\mathrm{x}$ flexibility $\mathrm{x}$ menu size on error rate. We found a significant effect of repetition $\left(F(5,90)=3, p=0.02, \eta p^{2}=0.14\right)$ and menu size $\left(F(2,36)=32.29, p<0.01, n_{p}^{2}=0.65\right)$ on error rate, however, did not find any main effects of flexibility or interaction effects.

\subsubsection{Repetition}

We proceeded with a pairwise comparison test with Bonferroni adjustments that revealed that the 1 st repetition $(10.5 \%)$ was significantly higher than the $5^{\text {th }}$ repetition $(7.9 \%)$, $p<0.01$. Although not significantly different the $1^{\text {st }}$ repetition averaged the highest error rate compared to all other repetitions (Figure 16). 


\subsubsection{Menu Size}

Our pairwise comparison for menu size found a significant difference for each menu pair where $p<0.01$ which suggested that an increase in items within a menu also increases error rate (small: $2.2 \%$, medium: $9.1 \%$ and large: $15.1 \%$ ).

\subsubsection{Item Location}

We performed a One-Way ANOVA investigating the effects of item location on error rate and found significant differences for the small, $F(3,42)=13.44, p=0.025, n^{2}=0.19$ and large, $F(11,154)=2.1, p=0.025, n_{p}^{2}=0.13$ menu sizes. We did not find any significance for the medium menu size.

We followed up with a pairwise comparison and found that within the small menu size, item 4 was significantly higher than items 1 and 3 where $p<0.01$. For the large menu size, we found that item 1 was significantly lower in error rate than items $2,4,5,8,9$, and 10 where $p<0.05$.

\subsection{Flexibility Preference Results}

After each completion of using a flexibility type and before starting the next set, we gave the participants a quick 3-Question Likert Scale questionnaire reflecting on their experience. Our goal is to obtain participants feedback just after they finished using that flexibility type. Our questions were aimed to understand if the pen was usable, responsive, and predictable. They were asked to provide a rating on their agreeableness. 


\subsubsection{Moved as Intended}

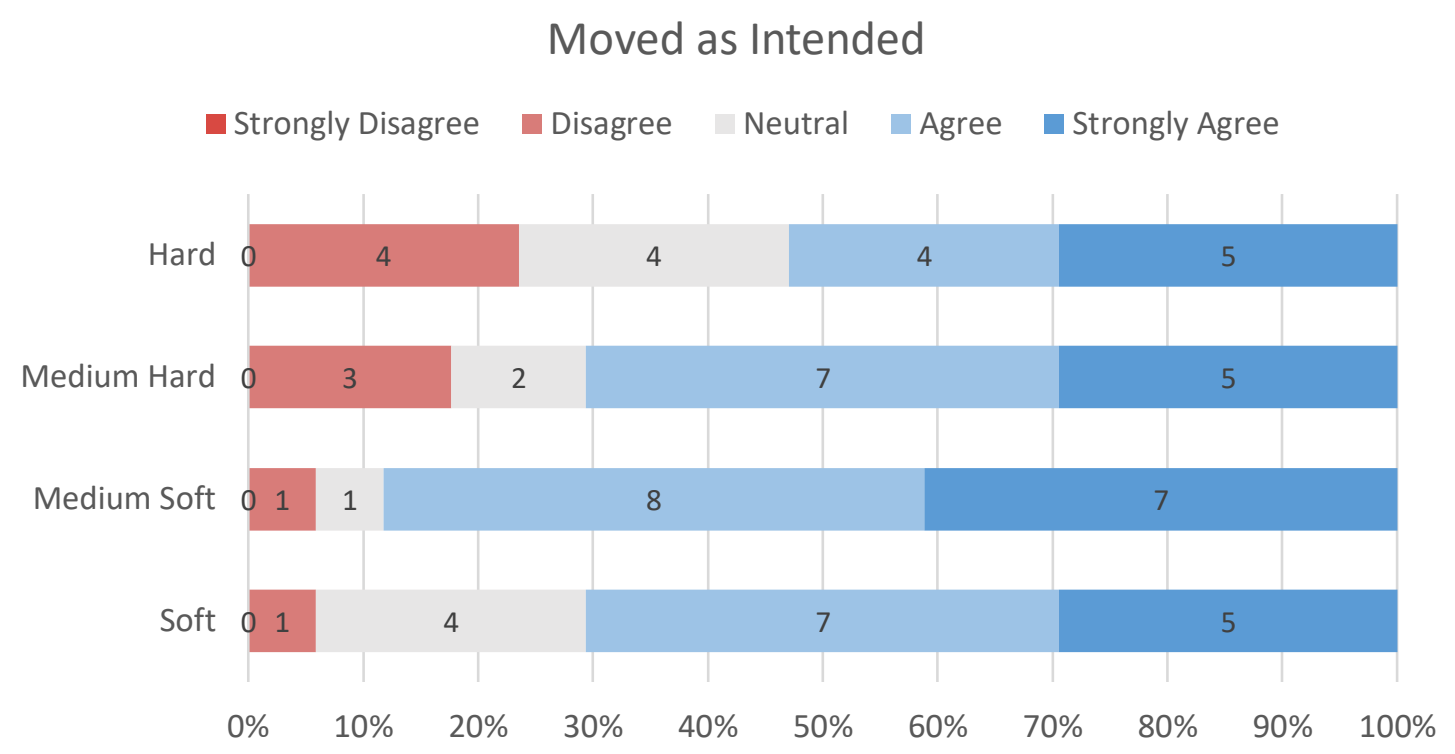

Figure 21 - Likert scale responses on the question, "Did the cursor moved as intended?"

Figure 21 represents the results asking if participants were able to move the cursor as they intended to. Majority of people Agreed/Strongly Agreed to the Medium Soft and Soft Flexibility type. Comparatively, more people voted to disagree with the Hard and Medium Hard Flexibility types. 


\subsubsection{Responsiveness}

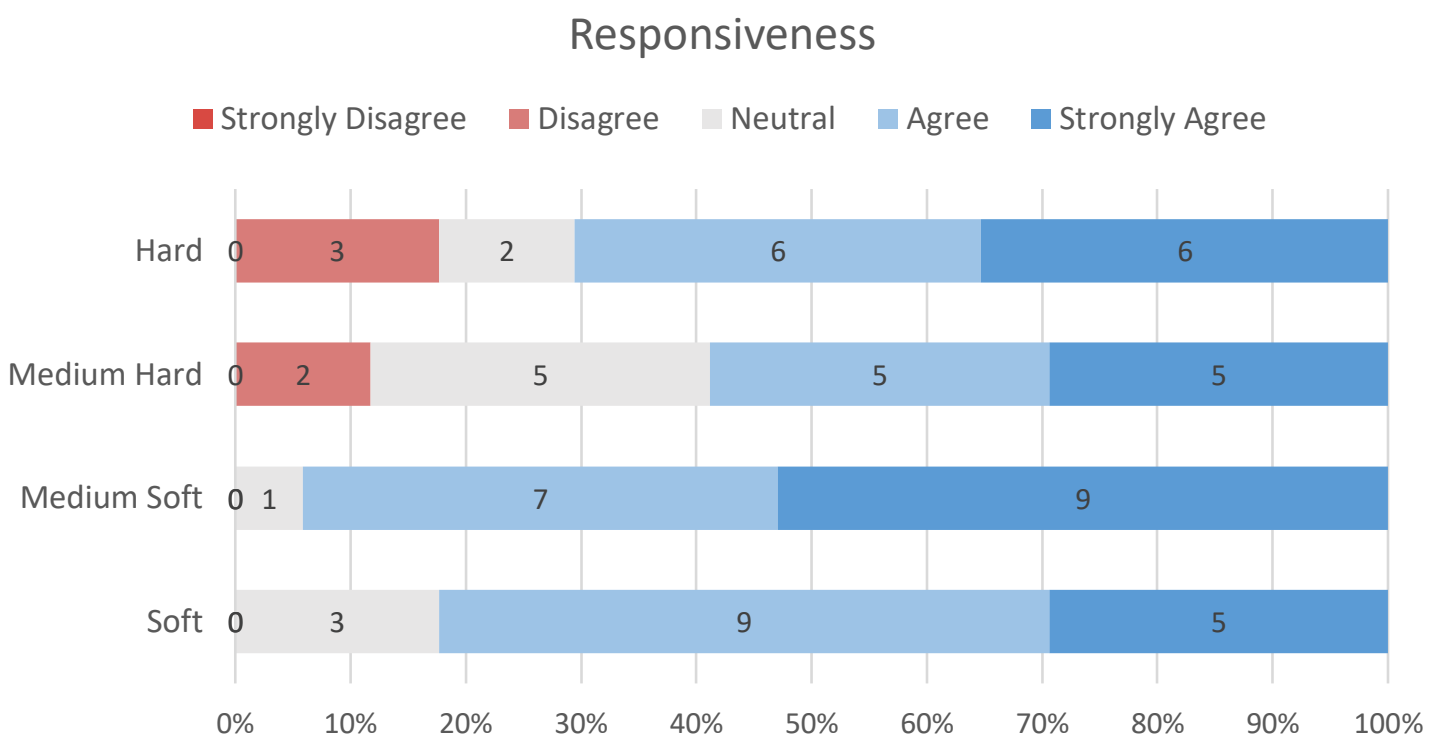

Figure 22 - Likert scale responses on the question, "How responsive was our device?"

Figure 22 shows participants Likert scale on how much they agree or disagree with the responsiveness of the device. Responsiveness is the delay between when the device receives the input from the user and the visual feedback given back to the user. If this delay is too long, it makes the prototype unusable or unfavoured. This can be due to inefficient programming or data being sent too quickly from the device to the application that it takes a noticeable amount of time for the application to parse the information in the queue before it can move on to the next set of data. As a result, this can show poor performances and latency issues. 


\subsubsection{Navigation}

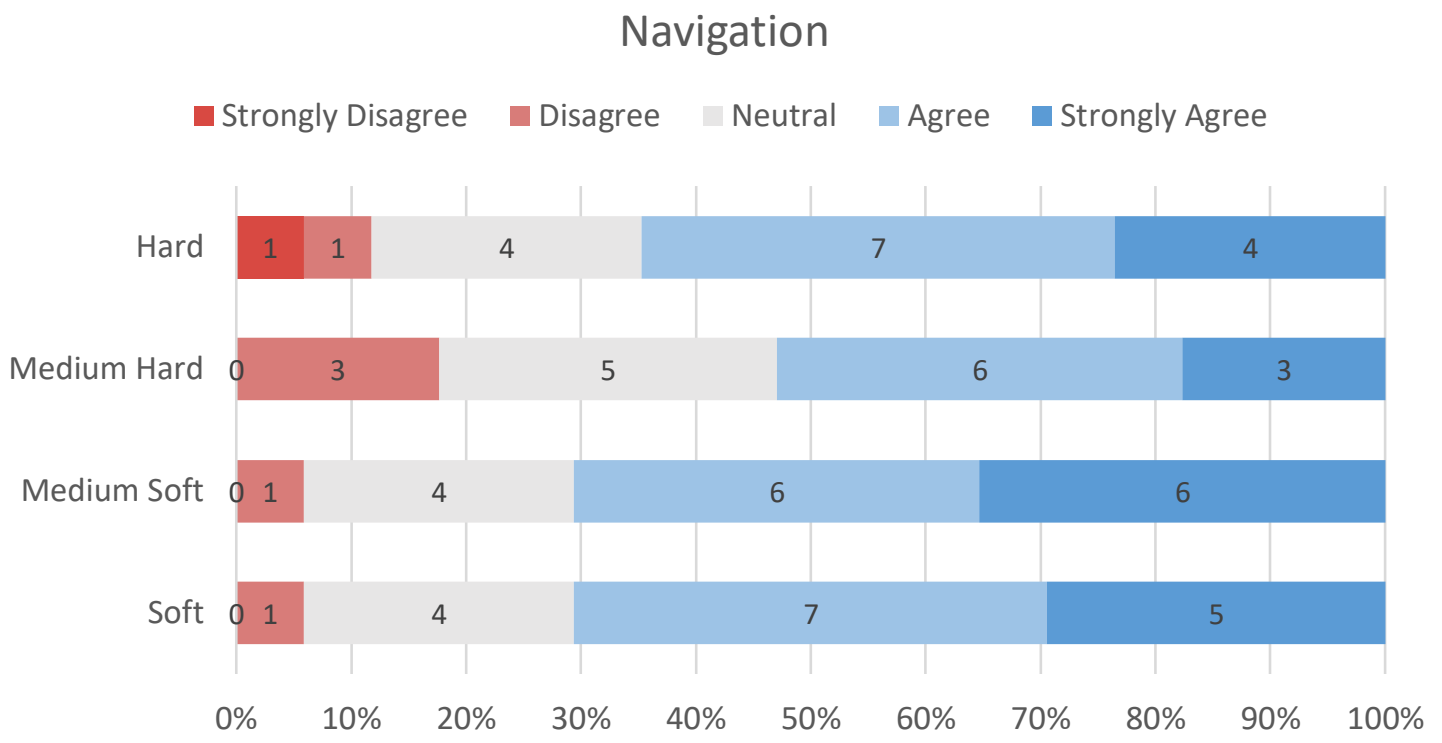

Figure 23 - Likert scale responses on the question, "Are you able to navigate to the target?"

Figure 23 outlines participants agreeableness ratings for their ability to navigate to the target correctly. This shows if participants were able to or not able to grasp our bending technique. We notice that results are fairly distributed across all four Flexibility conditions, however, one participant Strongly Disagreeing with the Hard condition and few disagreeing with the Medium Hard condition.

We performed a Wilcoxon signed rank test for each condition: Intention, Responsiveness, and Navigation to find any differences in perceived preferences. Our Wilcoxon Signed Rank test did not find any differences across the three conditions for each flexibility. We assumed that this could mean that the participants could not feel any differences in intention, responsiveness, and navigation for each of the flexibilities. 


\subsubsection{Flexibility Type Rankings}

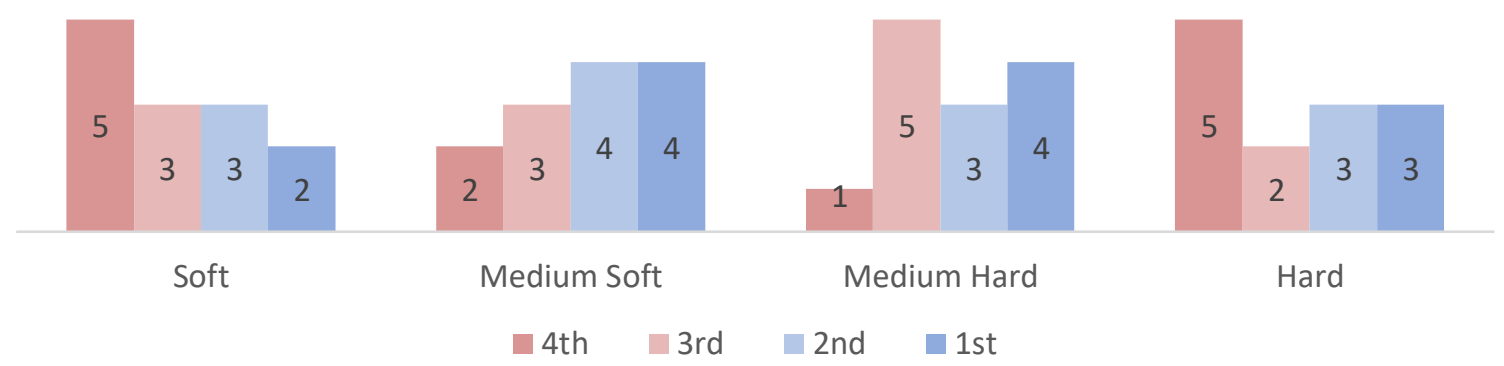

Figure 24. Total participants ranking from most $\left(1^{\text {st }}\right)$ to least $\left(4^{\text {th }}\right)$ preferred flexibility input type.

We had participants fill out a preference post questionnaire that asked to rank each flexibility type from most preferred to least. We gathered a total of $n=13$ rankings as we neglected 4 participants who misunderstood the question and only provided their most preferred flexibility. Figure 24 indicated that most people ranked the medium soft $(n=8)$ and medium hard $(\mathrm{N}=7)$ flexibilities as their $1^{\text {st }}$ or $2^{\text {nd }}$ preferred flexibility. While the hard and soft flexibilities had the most people rank $4^{\text {th }}$ ( $n=5$ for both).

P2, P3, P11, and P14, favored the harder flexibilities commenting that they felt they had more accuracy which lead to fewer unintentional movements. They also stated that the soft flexibility was too difficult to be precise when making small movements. Opposingly, P1, P6, P8, and P10, argued that the soft flexibilities were more responsive and easier to control. P6 and P10, also mentioned that the soft flexibility took the least amount of physical effort to move. $\mathrm{P} 5, \mathrm{P} 9, \mathrm{P} 13$, and $\mathrm{P} 16$, preferred the medium flexibilities as they stated that it was the perfect balance between the stylus being too soft or too flexible to control. 
P1, P5, P8, P9, P13, and P14, expressed that they admired the ability to quickly flick or point/bend towards an item without having to move the position of the stylus. We found this feature beneficial in a practical setting, users can quickly select items in a radial menu without having to disrupt their current pen position or mode. Interestingly, this was also found to be a feature of Tilt Menu where the user tilted the pen towards an item without having to lift off or move the stylus tip from its current position [57].

\subsection{Discussion}

We hypothesized that with an increase of items within a menu will also increase error rate (H1). From our analysis, we found this was correct for error rate and selection time as well. We hypothesized that an increase of flexural stiffness may result in higher error rates (H2). We also theorized that the stiffer materials, medium hard, and hard will be the least favoured (H3). Our subjective rankings did show that many people did not prefer the hard flexibility type $(n=5)$. Moreover, there was an equal amount that disliked the soft flexibility type $(n=5)$. Being there no distinct preferred flexibility type from our analysis, we could not conclude that users favoured the softer conditions for our HyperBrush device.

For a menu selection technique to work with bendable gestures, a menu size no greater than 8 items should be ideal. P7, P8, P10, P14, and P17, mentioned that it was difficult to select items that were located directly underneath their hand (south east areas). We advocate that menu tools of higher importance should be mapped to items located in areas that are not occluded by the users' hand to yield in best efficiency. For right-handed 
participants, we support that higher importance items should be placed in the south west or north west regions.

In spite of not finding a significant difference in preferred flexibility type, results still indicated that majority of people agreed to the softer flexibility being more responsive and moved as they intended. Participants also commented that they did not like the harder flexibility types because it required too much effort to control leading to muscle fatigue. This was also evident from observing the participants during the tasks. Opposingly, for participants that did not like the softer flexibility they stated that it was too flexible making it difficult to control and to be precise. The soft flexibility did not require a lot of physical exertion to bend and reach the maximum bend range, however, when it came to tasks where precision was critical, similarly the large menu size, this condition performed poorly.

Our interesting feedback revealed to us that the type of task may have an influence on what flexibility users favour. Many who preferred a stiffer HyperBrush, reasoned that they felt more control over executing fine and precise movements. While others who preferred a softer HyperBrush, stated that it did not require a lot of physical strength to move and being more comfortable to use.

\subsection{Limitations}

Bending our device does not require a lot of force, however, when consecutive repetitions occur, it can lead to hand strain or fatigue. We did not measure any effects of fatigue on accuracy although, it was evident from participants criticism. We emphasize that this 
should be considered during the protocol planning and longer breaks between tasks should be further encouraged.

We also asked the participants on the responsiveness and latency on the device. We noticed there were instances where the latency of bending the device and visualizing the movement of the cursor was too drastic that it could have affected performance. This resulted in us restarting and troubleshooting the device midway through experiments resulting in delay. We believed these issues were from transmitting an overflow of serial data from our device to the tablet causing in slow processing speeds. We accounted for these issues in study 2 $\& 3$ with a noticeable shorter difference in delay.

Our interface was reliant on the styluses orientation to be facing forward (directly north from the participant) at all times. This means that if the user regrips the device and rolls it dramatically within their hand, we lose calibration of our initial direction. It is difficult for the user to notice that they had rotated the stylus hence our implementation for the pseudobutton. To combat this issue completely, a gyroscopic sensor should be implemented to neutralize the rotational offset.

\subsection{Conclusion}

We evaluated the usability of a marking menu technique controlled by our HyperBrush's bendable input. Our results have concluded that an increase of menu size decreases performances. For a menu selection technique to work with bendable gestures, a menu size no greater than 8 items should be ideal. We advocate that menu tools of higher importance 
should be mapped to items located in areas that are not occluded by the users' hand to yield in best efficiency. For right-handed participants, we support that higher importance items should be placed in the south-west or north-west regions.

We did not find any effects of flexibility and this may be due to the small sample size used. Nonetheless, we are still interested in this examination for our subsequent study due to it being a completely different task that involved pen movement. For this, we decided to average the two (medium soft $\&$ medium hard) and reduce the number of flexible conditions to only soft, medium, and hard since the mean results from participant rankings being similar (2.23 for both).

When a device is too stiff, it is difficult to manipulate or deform that can lead to user fatigue or strain. When a device is also too rigid, there is a lot of force given back to the user to maintain or hold a position or gesture leading to user fatigue or strain. 


\section{Study 2: Brushstroke Width Manipulation}

\subsection{Introduction}

Our first study explored the usability of our HyperBrush for a bend menu selection technique that did not incorporate pen movement. Previous work compared stationary bend and pressure input for brushstroke width manipulation [18]. Our goal in the second study was to compare the ability to simultaneously control pen movement with bend or pressure

input. Specifically, we measured the performance (width and movement accuracy) of controlling bend and pressure input while moving on an X-Y plane simultaneously and compared it with the Microsoft (MS) Surface Pen, which includes pressure input. Carrying on from our previous study, we also incorporate measuring the influence of flexural stiffness.

\subsection{Methodology}

We investigated the influence of manipulating the brushstroke width by evaluating the user's performance on matching two target patterns: static and dynamic while moving in the given direction: North, South, East, West. This additional factor of moving in a direction goes beyond previous work [18], to provides a more comprehensive analysis of real brushstroke patterns, which are not always left to right (i.e. east). 


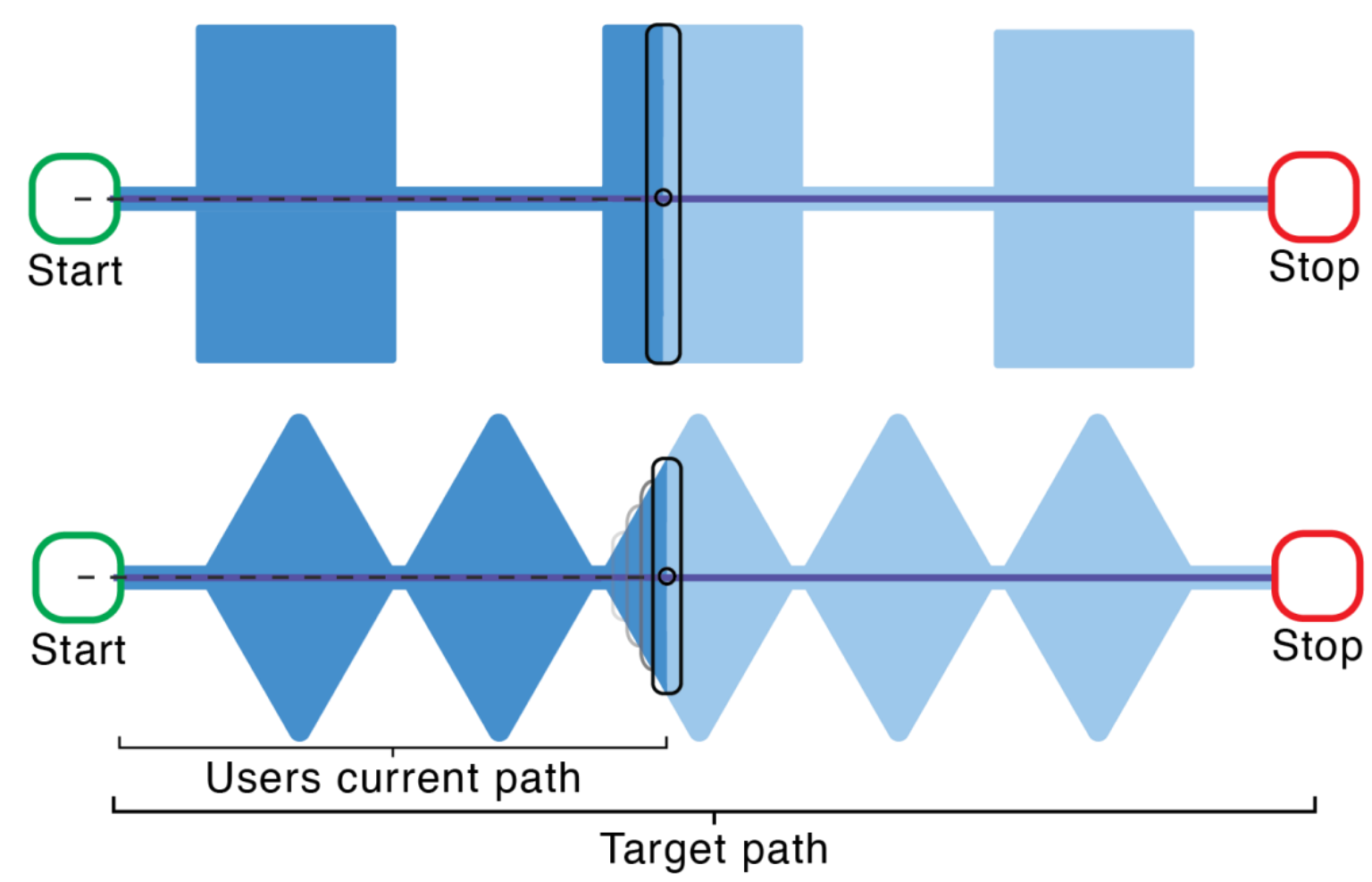

Figure 25. Brushstroke width manipulation task with the static (top) and dynamic (bottom) target patterns. Illustration of a user matching their path (dark blue) to the target path (light blue). The cursor (Black rectangle with a circle in the centre) indicates the current position and width size.

Static Target Pattern - As the user follows the path and its direction, they alternate between holding the maximum width of $4 \mathrm{~cm}$ and then the minimum width of $0.5 \mathrm{~cm}$ each for $10 \mathrm{~cm}$ of travelling distance.

Dynamic Target Pattern - User starts at the minimum width $(0.5 \mathrm{~cm})$ and increases the width to its maximum $(4 \mathrm{~cm})$ moving $10 \mathrm{~cm}$ amount of distance towards the given direction. Afterwards, the pattern then starts to decrease from the maximum width towards the minimum width and the cycle repeats. 
Before we start each task, we displayed only the start and stop indicators outlined in green and red as per Figure 25. We begin the task when the user places the stylus tip within the area of the green indicator. We end the task when the user enters the area of the stop indicator and then repeats presenting the next randomized task.

\subsection{Study Protocol}

We followed a within-subject design where participants used 4 inputs (soft, medium, hard flexibilities, and pressure) $\times 4$ directions (north, south, east, west) $\times 2$ target patterns $($ static and dynamic $) \times 5$ repetitions $=160$ trials that were randomized. Participants were prompted to rotate the tablet landscape for moving in the west/east directions and portrait for north/south directions. Between inputs, participants filled a Likert scale questionnaire regarding the input type.

We stored our target path in a comma-separated file (CSV) with 215 data points representing the path for the target. We assigned each point with a corresponding X-Y position on the path and a brushstroke width value that essentially displayed the target path. In order for us to measure the positional accuracy, we calculated the mean Euclidian distance between the target's points and the user's points, known as Proportional Shape Matching [2]. This method was also used to calculate the accuracy between a target and users set of points formed from a marking gesture $[2,31]$. Similarly, we calculated the width accuracy as a measurement of the absolute difference from the target and user widths. 


\subsection{Hypotheses}

H1. We hypothesized that the soft flexibility would perform better in width accuracy as opposed to the other flexibility conditions.

H2. We suspected that when decreasing in brushstroke width, our HyperBrush would perform better than the pressure stylus in width accuracy.

\subsection{Participants}

We recruited participants through email, social media, and posters. The majority were university students with no prior experience using flexible styluses. No participants were a part of the first study. We had 18 participants, 10 of which men and 8 women: 17 righthanded and 1 left-handed. These participants conducted study 2 and 3 in a single session (1h total), starting with the task in study 3 . We choose to present the studies that evaluated effects of flexural stiffness sequentially (study 1 and 2) and following, discuss the creative drawing task (study 3). The study was approved by our institution's research ethics board and we provided each participant $\$ 15$ compensation.

\subsection{Results}

We first decided to remove any effects of repetition on width \& positional accuracy and conducted a One-Way ANOVA for both. We found significant main effects on both width accuracy, $\mathrm{F}(4,64)=13.40, \mathrm{p}<0.01, \eta \mathrm{p}^{2}=0.46$ and positional accuracy, $\mathrm{F}(4,56)=22.95$ $\mathrm{p}<0.01, \eta \mathrm{p}^{2}=0.62$. A pairwise comparison with Bonferroni adjustment found a significant higher difference in accuracy from the $1^{\text {st }}$ to all other repetitions for width 
accuracy and $5^{\text {th }}$ to all other repetitions for positional accuracy, both at $\mathrm{p}<0.01$. Based on this result, we proceeded to disregard both the first and last $\left(1^{\text {st }}\right.$ and $\left.5^{\text {th }}\right)$ repetitions for the following analyses as we were not too particular on the learning effects of this study.

\subsubsection{Positional Accuracy}

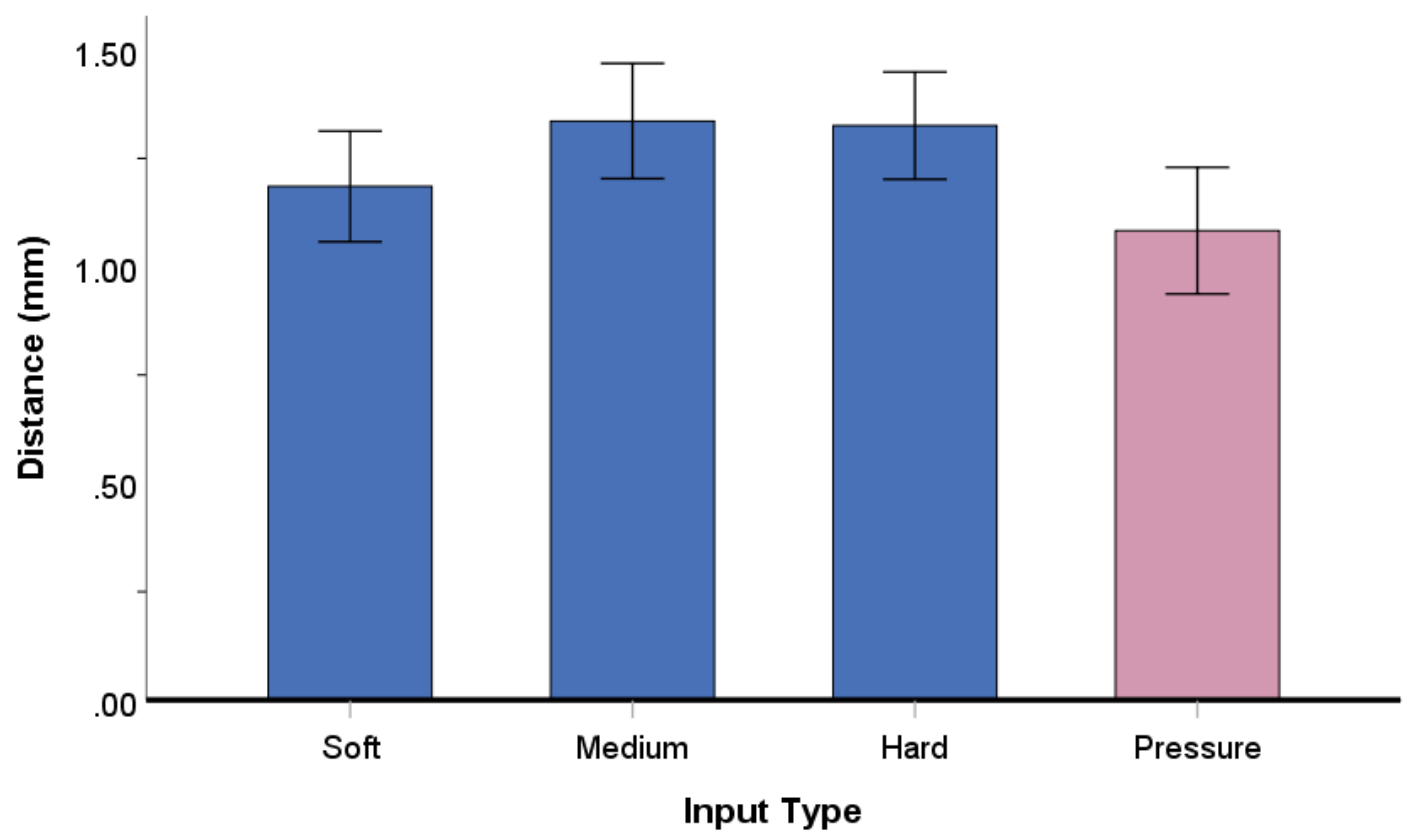

Figure 26. Positional Accuracy - Participants average X-Y distance from the target position for the three flexibility conditions and the pressure condition. 


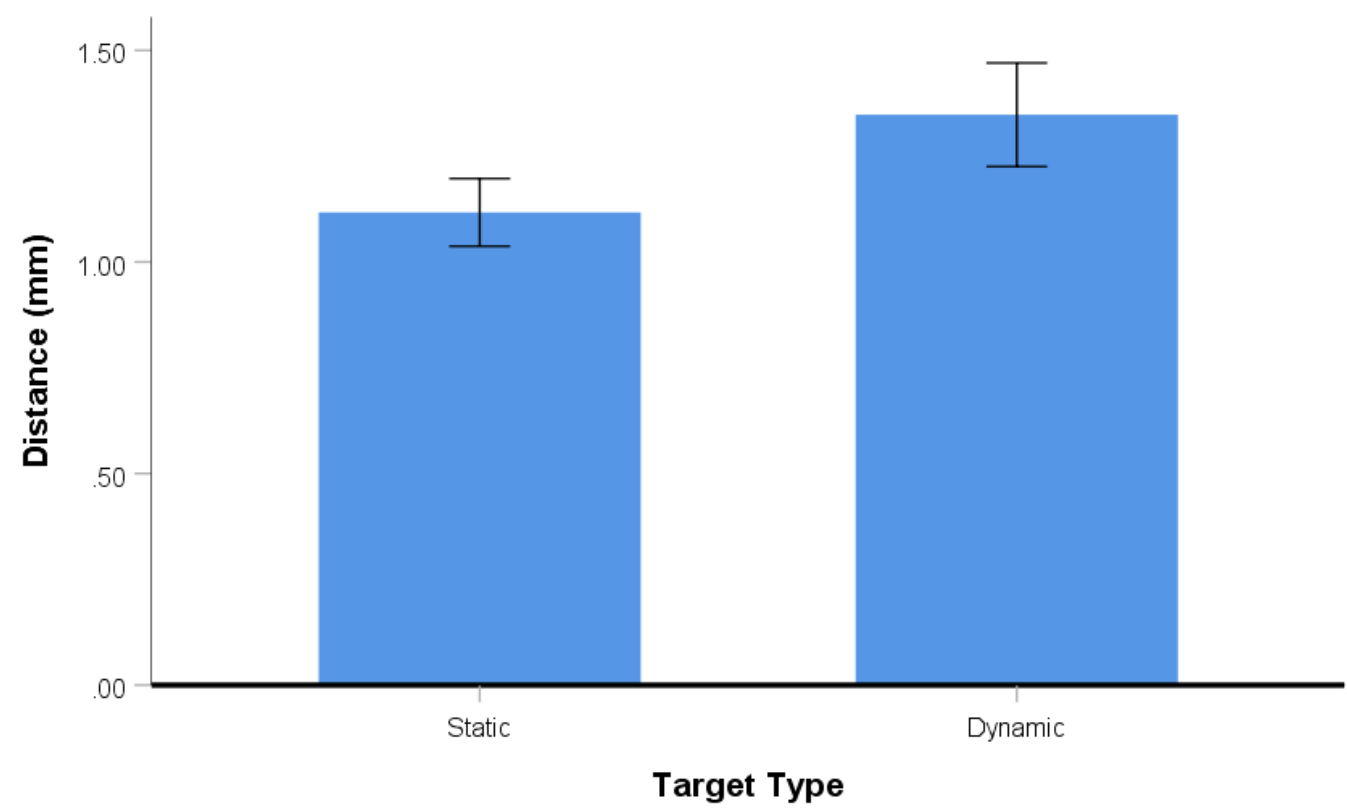

Figure 27. Positional Accuracy - Participants average X-Y distance from the target position for both target type.

We found that the soft flexibility was significantly lower than the medium flexibility, $p=$ 0.04. Interestingly, the baseline MS pressure pen condition was also significantly lower than the medium, $p<0.01$ as well as the hard, $p<0.01$ flexibility types. Being that the soft flexibility also yielded in the lowest average distance as opposed to the other flexibilities, this could suggest that the soft flexibility is easier to control the position of the stylus than the other flexibility types (Figure 26).

Our results found that the static target averaged in a significantly lower distance than the dynamic target, $p<0.01$ for positional accuracy. This could assume that users are more accurate with controlling the styluses X-Y position when not consistently changing the bending position of the device (Figure 27). 


\subsubsection{Width Accuracy}

We conducted a multi-way ANOVA across input type $\times$ direction $\times$ target type on width accuracy. Unfortunately, we did not find any main effects or interaction effects on any of the factors on width accuracy.

\subsubsection{Selection Time}

Lastly, we also conducted multi-way ANOVA for Selection Time and found a significant effect of direction on completion time, $\mathrm{F}(3,45)=3.8, p=0.02, \eta \mathrm{p}^{2}=0.34$ and no further interaction effects were found. We followed with pairwise comparison test using Bonferroni adjustment and revealed that moving in the east direction (31s) yielded in significantly lower average completion time than moving in the west direction (37s) where, $p=0.04$. Also, we found that moving towards the north direction (32s) was also significantly lower than moving in the south (35s) and west (37s) directions where $p=0.4$ and 0.1, respectively. We illustrate this relationship below in Figure 28.

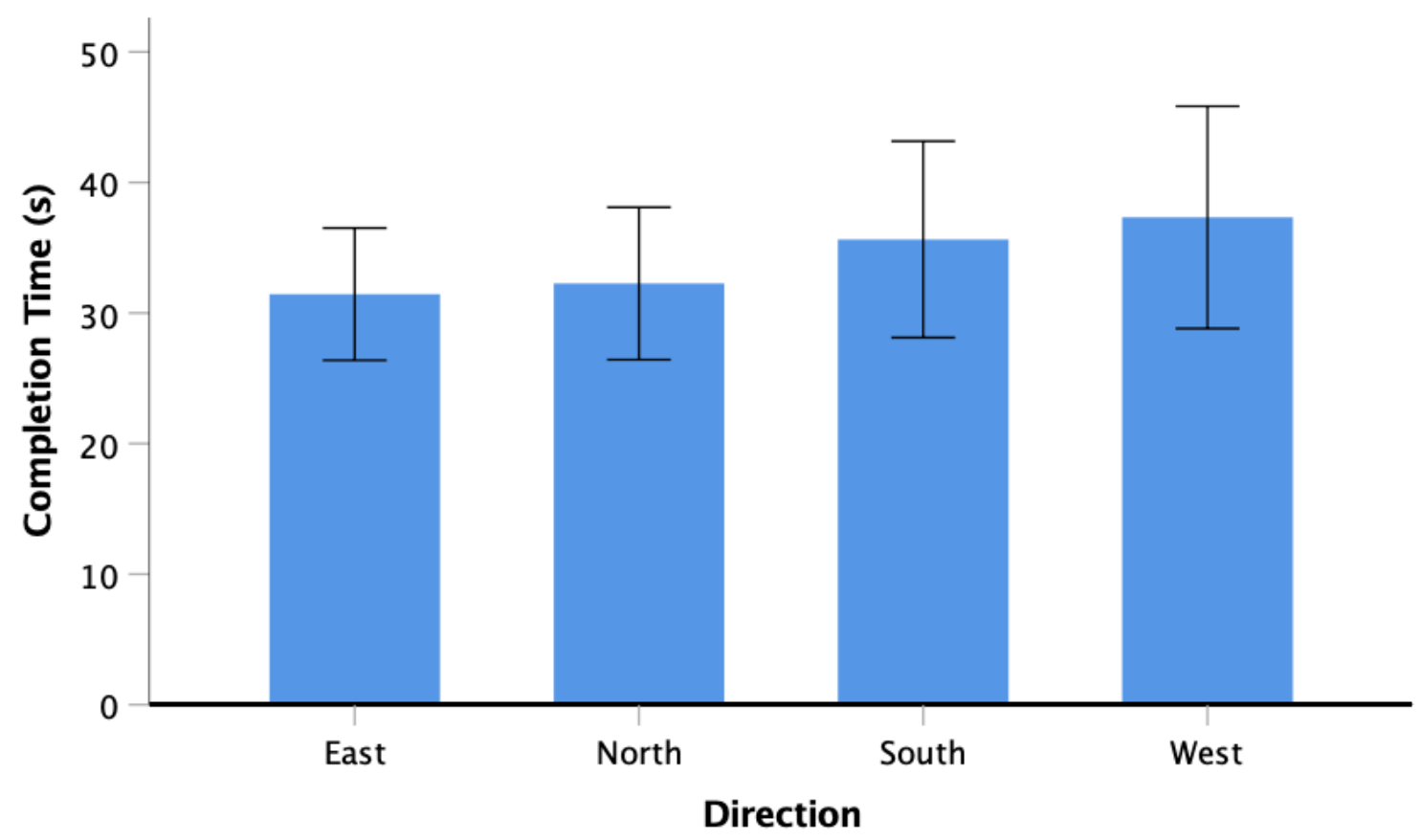


Figure 28. Average completion times for each direction with $95 \%$ CI.

\subsection{Flexibility Preference Results}

We performed a Wilcoxon Signed Rank Test on the three conditions: Intention, responsiveness, and navigation. We did not find any significant differences. Coincidentally, these results are on par to our results from our first study where we also did not find any differences.

\subsubsection{Moved as Intended}

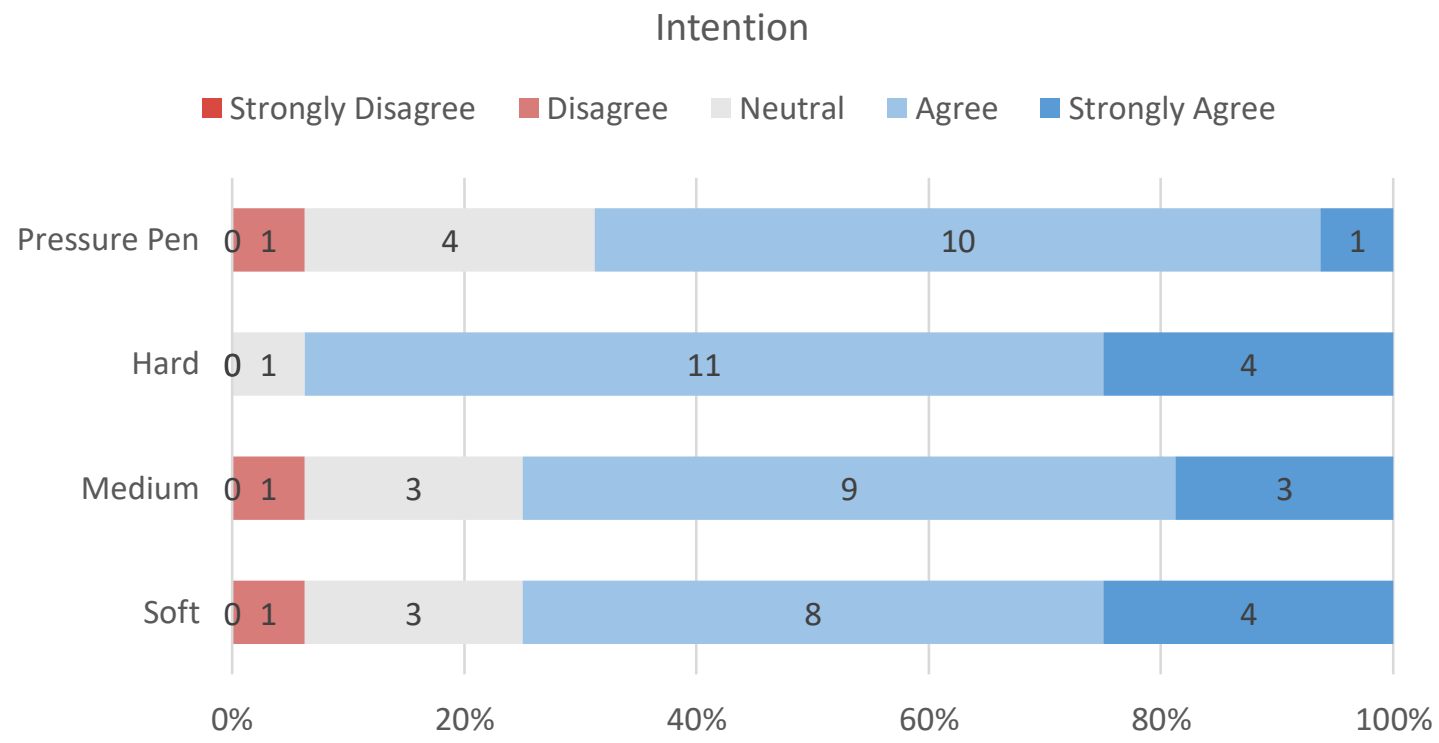

Figure 29. Likert scale responses on the question, "Did the cursor moved as intended?"

We found that most participants either agreed or strongly agreed with the hard flexibility input type $(\mathrm{n}=15)$, soft/medium at second most $(\mathrm{n}=12)$, and our baseline pressure input type the least $(\mathrm{n}=11)$ (Figure 29). 


\subsubsection{Responsiveness}

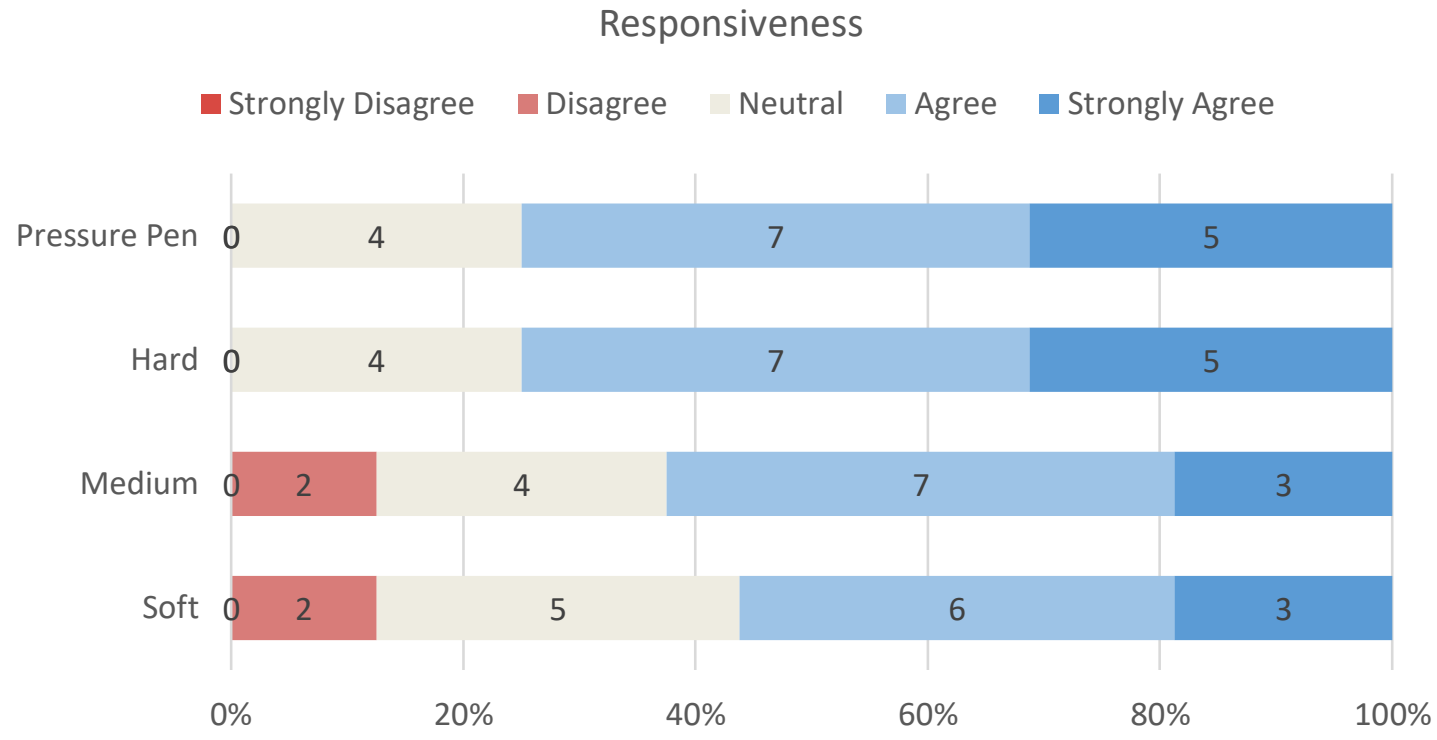

Figure 30. Likert scale responses on the question, "How responsive was our device?"

From our results in Figure 30, the hard flexibility and pressure input types each had $n=12$ participants in total agree or strongly agree as being responsive. Moreover $\mathrm{n}=0$ participants rated lower than neutral as compared to both the soft and medium flexibilities where $\mathrm{n}=2$ for both. 


\subsubsection{Navigation}

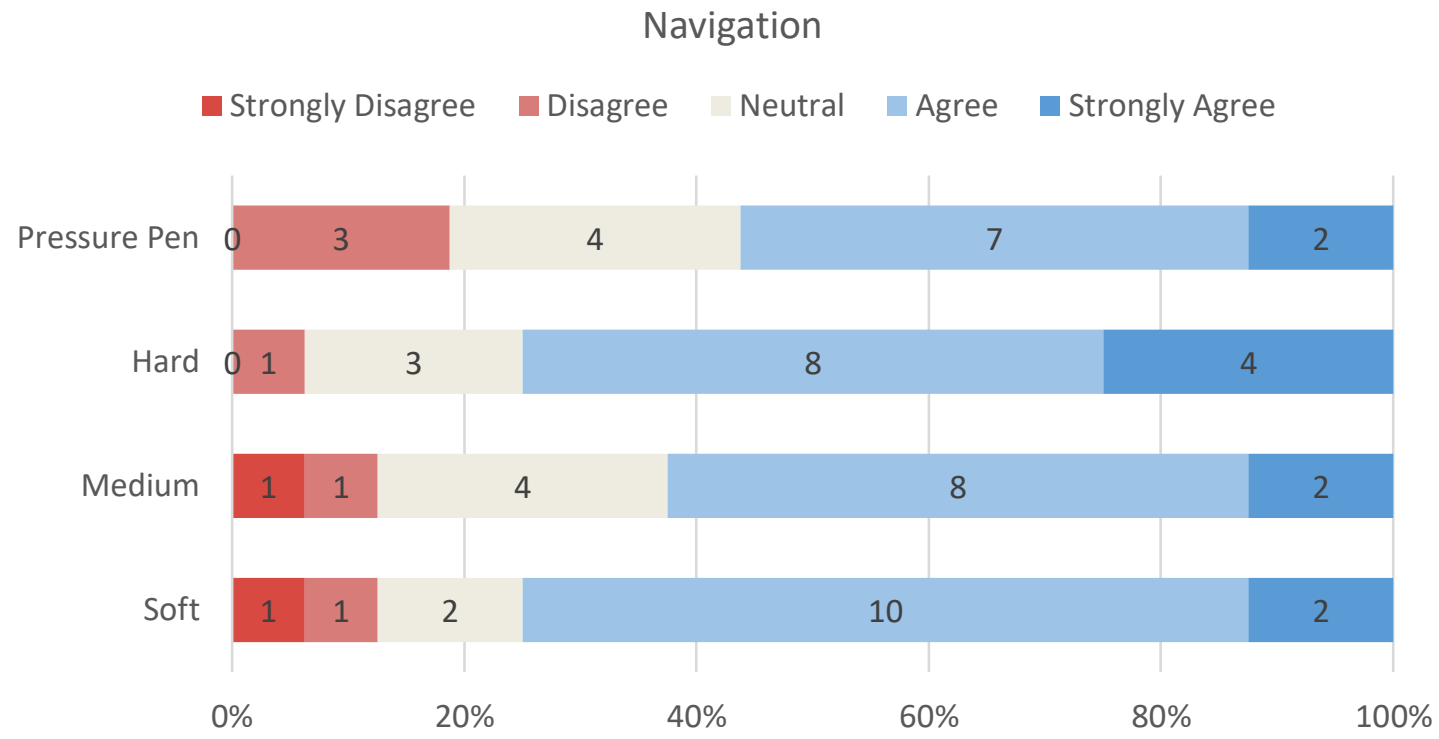

Figure 31. Likert scale responses on the question, "Are you able to navigate to the target?"

Figure 31 shows that most people either agreed or strongly agreed with the soft and hard flexibility types at $n=12$ for both while the pressure input type had $n=3$ participants disagreeing with their ability to navigate.

\subsubsection{Flexibility Type Rankings}

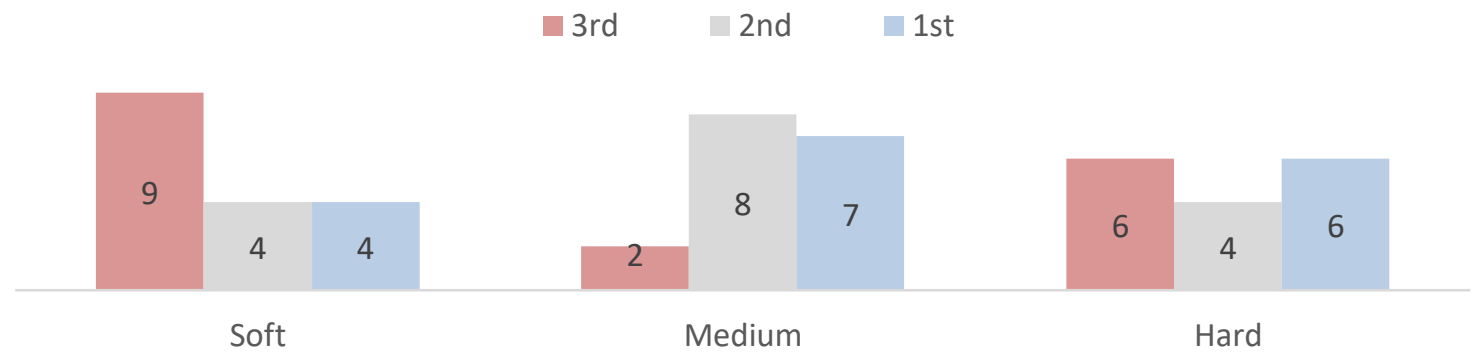

Figure 32. Summary of participant rankings from most $\left(1^{\text {st }}\right)$ to least $\left(3^{\text {rd }}\right)$ preferred flexibility input type. 
Similar to the first study our Wilcoxon Signed Rank Test on flexibility preference yielded in no significant results. However, the medium flexibility resulted in the most preferred mean score of 1.7 while the soft and hard flexibilities were 2.3 and 2.0, respectively (Figure 32).

\subsection{Discussion}

We hypothesized that the soft flexibility would perform better in width accuracy than the other flexibility types (H2). We did not find any significant differences between flexibility type for width accuracy. From looking at how users perceived the performances for each flexibility, it may also be inconclusive to approximate a dominant flexibility type.

Looking back at our marking menu study, we also did not find any effects from flexibility. We found these results interesting as we speculated that flexibility could depend on the user's personal preference. With our HyperBrush, users have the option to interchange components to their preferred flexibility or based on the current task being executed. For example, to reiterate we found that people liked the stiffer materials for smaller precision tasks, in this case writing tasks could benefit. As for a softer material, drawing or sketching might be valuable here. As for drawing or sketching, we think that the softer material might be of value for these scenarios that require quick gestures and strokes. Commercial versions of HyperBrush could either be a set of several stiffnesses brushes, or a single brush with interchangeable parts of different flexibilities, including a rigid one. 
We also hypothesized that the static target pattern will produce better positional accuracy as opposed to the dynamic pattern type (H3). From our analysis, we found that the Euclidian distance between the user and target positions were significantly smaller in the static pattern type compared to the dynamic pattern type. We believe that because the dynamic pattern required the user to constantly increase/decrease the width amount, thus making it difficult to control the position of the stylus.

We noticed two main scenarios where the bending direction of the HyperBrush was the same as the moving or opposing direction. For example, if the participant was moving leftward, they would also bend HyperBrush towards the left or opposite, right. We denoted this as either dragging - where the tip is being dragged behind or pushing - the tip is in front being pushed from the back. Although we did not restrict or control the direction of bend for this study, this could be an interesting factor to also consider for future work involving flexible styluses.

\subsection{Limitations}

Our HyperBrush had a larger pen tip surface area $\left(28 \mathrm{~mm}^{2}\right)$ as opposed to the Microsoft Surface pressure pen $\left(0.80 \mathrm{~mm}^{2}\right)$, which made it difficult for users to make very fine (small) brushstrokes. Additionally, HyperBrush's tip was made from a rubberized compressible material opposed to the matte plastic texture from the MS pen therefore making it difficult to compare the feeling produced when drawing. We chose a larger pen tip to provide a sufficient amount of frictional force to avoid accidental slippage when bending the device. We believed that the MS pen being a commercialized product had the performance 
advantage due to its pressure transfer functions being thoroughly calibrated for this intention. If that is the case, our comparable results between a prototype and a commercial product could be interpreted as something positive.

We explored a range of flexural stiffnesses for our HyperBrush, from $0.1 \mathrm{~N} / \mathrm{mm}$ to $0.139 \mathrm{~N} / \mathrm{mm}$ yet neither study found any influence on users' performance. Considering only that a total range difference of $0.039 \mathrm{~N} / \mathrm{mm}(4 \mathrm{~g} / \mathrm{mm})$ could potentially be too narrow to yield any significant differences. While it would have been ideal to explore a larger range differences, $0.08 \mathrm{~N} / \mathrm{mm}(7.14 \mathrm{~g} / \mathrm{mm})$ for example, we were constrained by the minimum amount of wall thickness without compromising the robustness of the device and fitting a sensor within its cavity. We also consider that participants also had prior exposure with using the medium flexibility during study 3 which may have an additional learning effect.

\subsection{Conclusion}

We explored participants performance of simultaneously controlling pen movement and bend or pressure input. We measured the effects of flexural stiffness with three flexibility conditions (soft, medium and hard) and similarly to Study 1 we did not find any effects. We also could not conclude if there is a distinct preferred flexibility, however, we speculate that flexibility maybe a matter of personal preference. 


\section{Study 3: Creative Digital Drawing Task}

\subsection{Introduction}

In this study, we sought to assess how our HyperBrush can be a supportive tool for users' creativity. We were also interested on how it performs compared to a commercially available pressure sensitive stylus, the Microsoft Surface Pen. We used the same set of participants complete this study prior to executing study 2. To reiterate, majority of participants had no prior experience to using flexible styluses and little experience using digital styluses.

\subsection{Methodology}

We developed our own free form digital drawing application that supported input from both stylus types. We programmed our application using Unity Engine and coupled both absolute bend and pressure input to vary the width of the brushstroke. For instance, a larger bend or pressure input resulted in a larger brushstroke width. For the flexible input, we decided to only use the medium flexibility $(0.129 \mathrm{~N} / \mathrm{mm})$ condition, being the combination of both hard and soft extremes.

We followed a study design similar to Aslan et al. that instructed participants to draw an illustration for both input types [5]. We instructed participants to draw two illustrations (cityscape and landscape), one per input type (bend and pressure), in which the order was reversed per participant. We gave participants 10 minutes for each illustration and was also encouraged to think aloud. We hypothesize that our participants would perceive 
HyperBrush to support creativity more than a pressure stylus (H1). To address H1, we had participants fill out a Creative Support Index (CSI) questionnaire that examined their subjective perception of how each stylus type can support the five dimensions of creativity (Enjoyment, Expressiveness, Exploration, Results Worth Effort, and Immersion) [13]. The 18 participants in Study 3 also completed study 2.

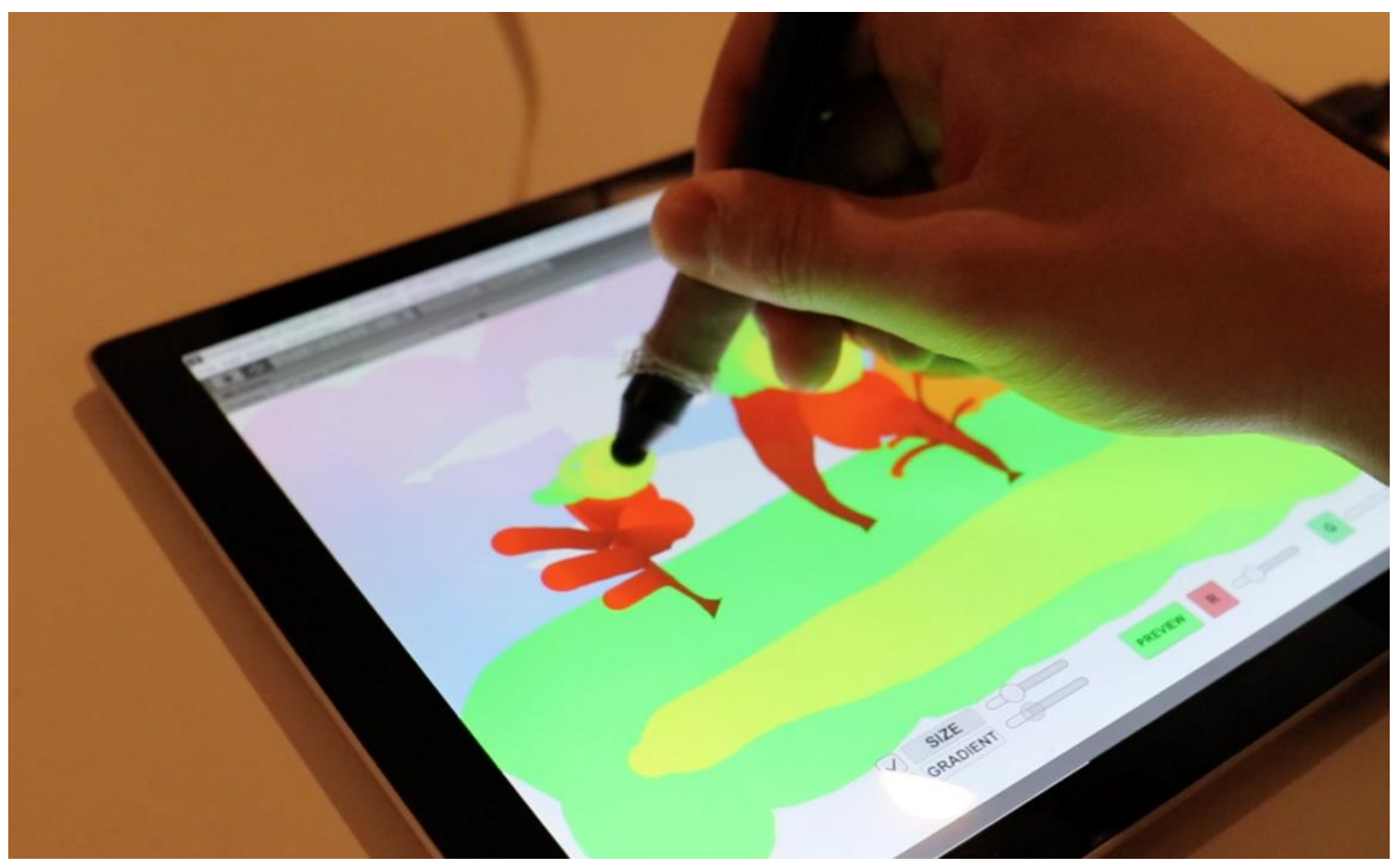

Figure 33. Drawing Application with HyperBrush.

\subsubsection{Application Interface Design}

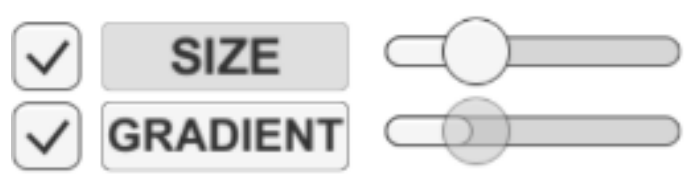

Figure 34. Menu buttons for Brushstroke Size Modifier (Active) and Gradient Toggle with Modifier (Inactive).

Brushstroke Size Modifier - Size modifier amplified the default brushstroke width size by multiplying the width by a variable value from the slider input (Figure 34). 
Gradient Toggle - Applied a gradient effect of $[0,255]$ to a given colour. This feature was available, however, not utilized by the majority of the participants (Figure 34).

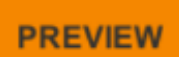

$\mathbf{R}$

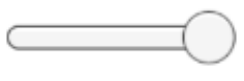

B

Figure 35. Interface for the Colour Modifier Toolbar.

Colour Modifier - Three individual RGB colour sliders ([0, 255], [0, 255], [0,255]) with a preview window displaying the current colour being used. Though, a colour palette selector interface may have been more ideal in this scenario, however, due to programming and time restrictions this was our best implementation (Figure 35).

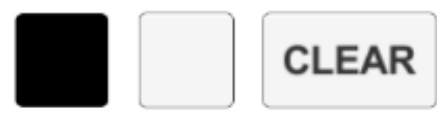

Figure 36. Menu shortcut buttons for black, eraser, and clear.

Black Selection - Dedicated selectable button to change the current colour to black (Figure 36).

Eraser (White Selection) - Dedicated eraser function that changed the current colour to an eraser tool (white) (Figure 36).

Clear - Clears and deletes all strokes to an empty canvas (Figure 36). 


\subsection{Creative Digital Drawing Results}

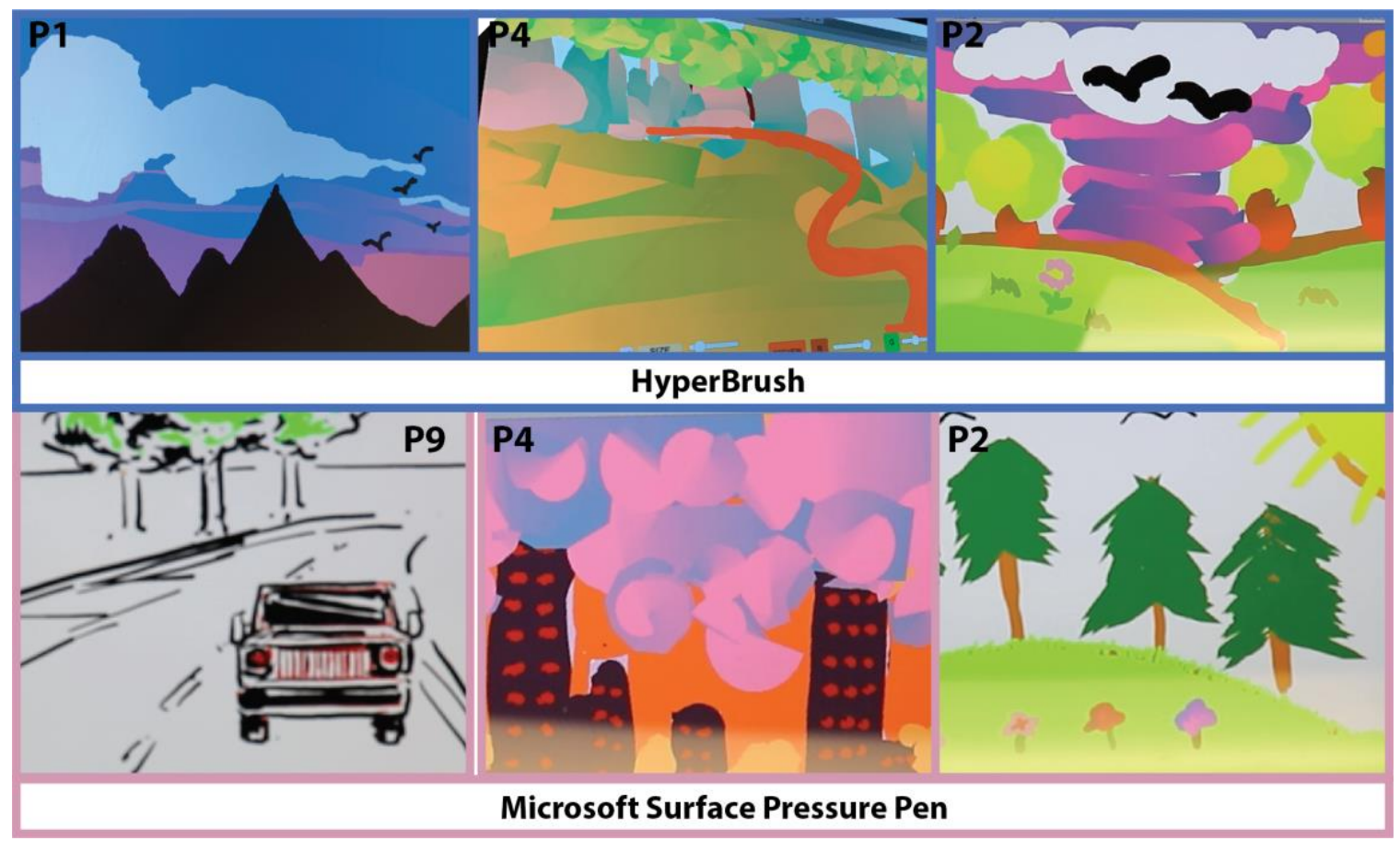

Figure 37 - Collage of participants' drawings of a landscape or cityscape from using both styluses.

Figure 37 illustrates P1, P2, P4, and P9 drawings from both input and illustration types. Overall, most illustrations produced by our HyperBrush exhibited larger brushstrokes that was ideal for drawing large patches of land, grass, and sky. There was also a trend of participants drawing skies with colorful sunsets taking advantage of the gradient tool. On the other hand, the MS pressure stylus revealed drawings of smaller strokes detailing thin outlines, birds, small bits of grass, and windows.

P1, P2, P12, P17, and P18 expressed that the HyperBrush acted similarly to a paintbrush. We believe that this is because the flexible barrel bends in the same fashion to how the bristles on a paintbrush bends, it curves as the tool is dragged on the surface. P5, P14, and P16 also mentioned that the visual affordance from our HyperBrush helped them control 
the amount of bend due to being able to see the curvature as well feeling the resistive feedback from the flexible barrel.

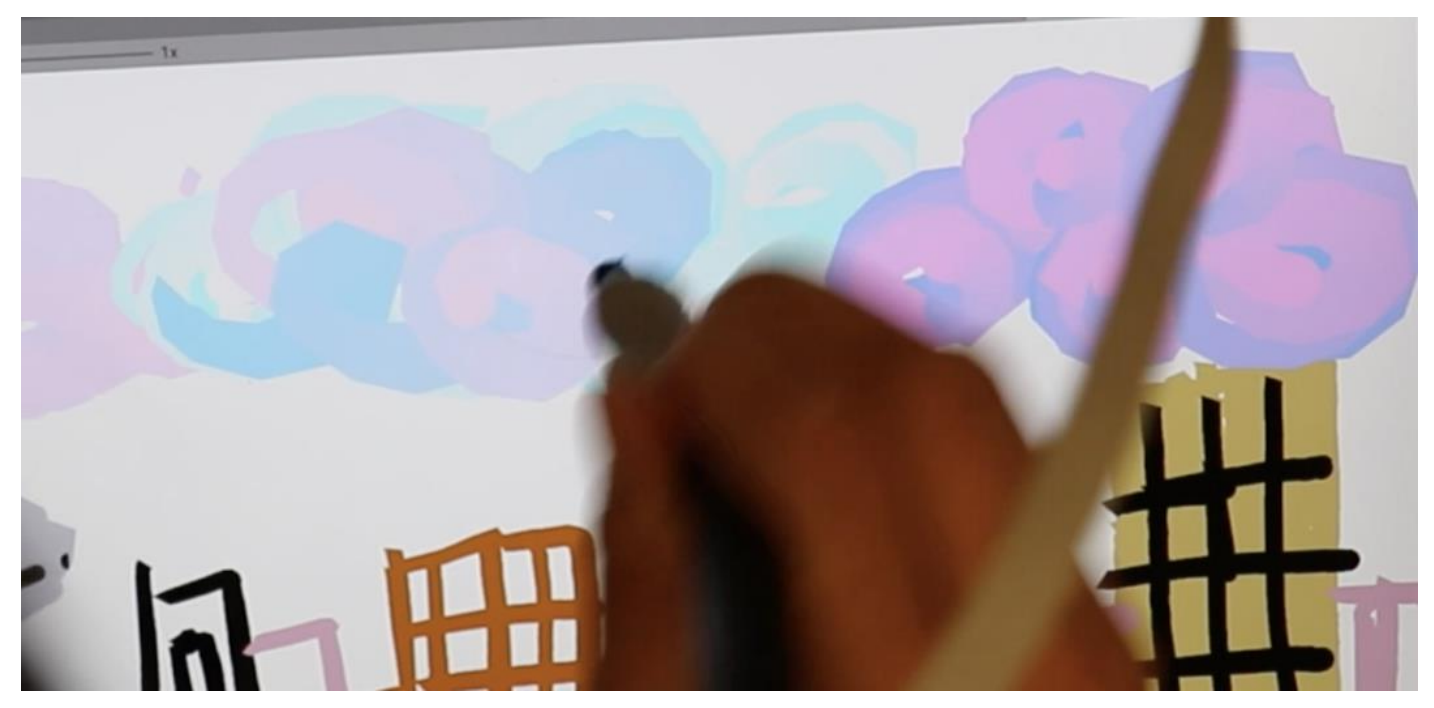

Figure 38. Example of $P 12$ drawing colorful gradient clouds with using repetitive circular motions while bending and rotating the HyperBrush.

P12 commented on how they enjoyed performing a circular motion technique with both styluses, in which the gradient effect added color depth to objects such as clouds and treetops (Figure 38). P12 explained that they bent the device while directionally pointing it in a rotational fashion creating colorful gradients and visually appealing cloud like figures. P4 and others have done a similar technique while drawing their own clouds as well as treetops.

We observed that participants held the HyperBrush low on the shaft with the intention to prevent any unintentional bending and continue to use it as a regular stylus. P5 emphasized that it felt unusual gripping the HyperBrush directly over the flexible point as it felt too soft compromising a sturdy grip. 
P2 and P16 expressed the same concern with the difficulty of changing directions while maintaining bend input. P2 also argued that this could be a feature like painting Chinese calligraphy where directional changes can add a unique style to the size of the brushstroke width. In this case with some experience and learning, directional changes can be used to the user's advantage to produce smooth, intentional, and artistic, variants in brushstroke width.

\subsubsection{Creative Support Index}

To analyze how users perceived HyperBrush as a tool can support creativity, we calculated the CSI scores for our HyperBrush and used the MS pressure pen as the baseline. Figure 39 shows that the average perceived creative support across all dimensions were similar for both input types. Interestingly, our HyperBrush's average perceived creative support was similar to the baseline condition. We conducted a Wilcoxon Signed Rank Test for each dimension of creativity and the overall CSI score and strangely did not find any significant differences between our HyperBrush and the baseline.
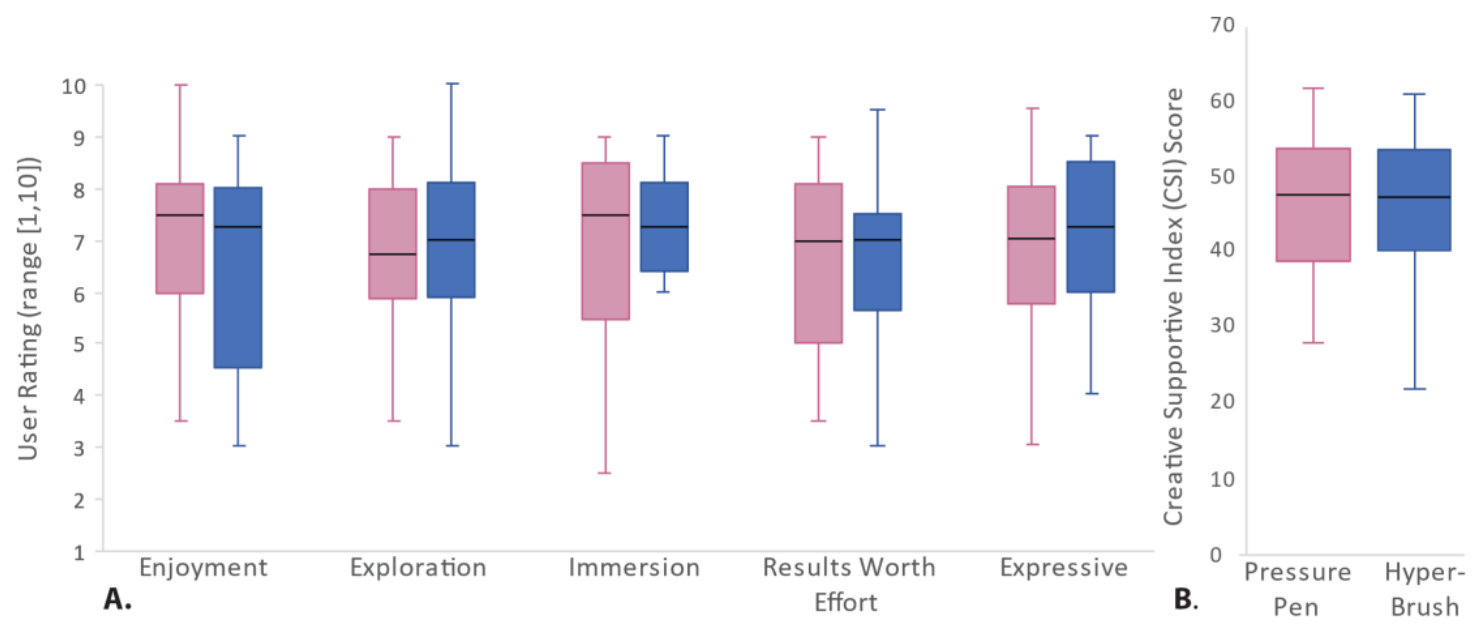

MS Pressure Pen $\mathbf{H y p e r B r u s h}$ 
Figure 39. A. Perceived average Likert scale ratings with SD for each dimension of creativity and B. Calculated Creative Support Index (CSI) scores with SD for our HyperBrush and the baseline condition MS Pressure Pen.

\subsection{Discussion}

We hypothesized that users would perceive our HyperBrush to be a greater tool to support creativity as compared to the pressure stylus (H1). Our participants felt that using our HyperBrush, they could express themselves more creatively as opposed to using the pressure stylus. For all other dimensions of creativity and overall CSI score, we did not find any significant differences, thus we cannot conclude that our HyperBrush can foster greater overall creativity. Although we did not find any differences in supporting creativity between our HyperBrush and the pressure stylus, most participants preferred our HyperBrush $(n=13)$ over the pressure pen $(n=5)$ as they stated it was more enjoyable for them to use. We speculate the reasoning behind this is that many participants referred to our HyperBrush similar to using a digitalized paint brush. Few commented that the curve created from bending was almost resembling the bristles of a paintbrush being dragged against the surface.

\subsection{Limitations}

Our HyperBrush had a larger pen tip surface area $\left(28 \mathrm{~mm}^{2}\right)$ as opposed to the Microsoft Surface pressure pen $\left(0.80 \mathrm{~mm}^{2}\right)$, which made it difficult for users to make very fine (small) brushstrokes. Additionally, HyperBrush's tip was made from a rubberized compressible material opposed to the matte plastic texture from the MS pen therefore making it difficult to compare the feeling produced when drawing. We had chosen to have a larger pen tip area to provide a sufficient amount of frictional force to avoid accidental slippage when 
bending the device. Because of these differences, we suspected that this could have had an influence on the user's performance. We believed that the MS pen being a commercialized product had the performance advantage due to its pressure transfer functions being thoroughly calibrated for this intention. Overall, this may be why users were more comfortable with the MS pen.

Our participants were recruited from the general population through email, social networking, and posters, of which not many considered themselves as artists or experienced using digital stylus interfaces. Although we obtained valuable feedback from our participants, a study with a more targeted population could reveal different results.

As mentioned before, participants experimented with the HyperBrush's bending direction as we did not constrain this factor. We think that this could have ultimately affected performance data as participants were still trying to figure out a comfortable direction to bend the device. 


\section{Conclusion \& Future Work}

We presented HyperBrush, a digital stylus capable of utilizing bendable input as an additional degree of freedom for pen-based applications. We designed HyperBrush to have interchangeable flexible components so that the stiffness of the device can be accommodated to the user's preference and task. We conducted two studies to evaluate HyperBrush, one assessing the performance on a menu technique and the second comparing bend and pressure when controlling brushstroke width while moving the pen.

We evaluated the usability of a bend marking menu technique and recommend that items should be placed in the south-west region as opposed to the occluded south-east region for right-handed users. For a bend menu technique, we found that a menu size no greater than 8 items is ideal. From participants feedback, they enjoyed using our HyperBrush device and thought that the idea of quickly flicking the bend towards an item was fast and effective. We obtained majority of right-handed participants therefore we were not able to gather enough data to conduct an analysis on left-handed users. It would also be interesting to investigate the effects of item location on left-handed users and compare our results to see if there is a symmetrical outcome involved.

We observed how HyperBrush can perform similarly to a commercial pressure pen when it came to supporting users' creativity and controlling brushstroke width while moving. Our first two studies evaluated the effects of flexural stiffness on users' performances. While our analysis did not find any influence of flexural stiffness on users' performance or preference, participants feedback showed that the stiffness of a flexible stylus could be of 
user's personal preference. We believe that HyperBrushs' variety of flexibility is analogous to having a wide selection of paintbrushes available for an artist, each flexibility or brush providing their own distinct advantages and style to the artist.

For future prototyping of a flexible sensing stylus, we recommend exploring different pen tip sizes and materials. We think that a smaller pen tip size that does not compromise the ability and control of bending could potentially expand this research field into other applications such as digital writing or object manipulation that demands precision of the pen tip.

It would be interesting for further work to look into an active HyperBrush type where a mechanism within the stylus can change the flexibility properties by adding or reducing the amount of flexibility prompted by the user with just a turn of a knob integrated on the stylus-top barrel. We think this could improve the seamlessness between changing stiffnesses because there would be no need to swap out any components or hardware.

Although research on a bendable stylus is still preliminary, we believe that there is a lot of potential to expand in studying its capabilities as an alternative pen input technique. In upcoming work, we look forward to an in-the-wild, longitudinal study with digital creative artists or designers to understand how HyperBrush could be a tool to better support their creative applications. We also hope to implement a comprehensive set of HyperBrush prototypes ready to go similarly to a different pencil type in a pencil case. 


\section{Bibliography}

[1] Ahlström, D., Cockburn, A., Gutwin, C. and Irani, P. 2010. Why it's quick to be square: Modelling new and existing hierarchical menu designs. Conference on Human Factors in Computing Systems - Proceedings. 2, (2010), 1371-1380. DOI:https://doi.org/10.1145/1753326.1753534.

[2] Andersen, T.H. and Zhai, S. 2010. Writing with music: Exploring the use of auditory feedback in gesture interfaces. ACM Transactions on Applied Perception. 7, 3 (2010). DOI:https://doi.org/10.1145/1773965.1773968.

[3] Annett, M. and Anderson, F. 2014. The pen is mightier: Understanding stylus behaviour while inking on tablets. Proceedings of Graphics Interface 2014 - GI 2014 (2014), 193-200.

[4] Arduino Mega: https://store.arduino.cc/usa/mega-2560-r3.

[5] Aslan, I., Weitz, K., Schlagowski, R., Flutura, S., Valesco, S.G., Pfeil, M. and André, E. 2019. Creativity Support and Multimodal Pen-based Interaction. 2019 International Conference on Multimodal Interaction (New York, NY, USA, Oct. 2019), 135-144.

[6] Bailly, G., Lecolinet, E. and Nigay, L. 2008. Flower menus. Proceedings of the working conference on Advanced visual interfaces - AVI '08 (New York, New York, USA, 2008), 15.

[7] BendLabs Inc.: https://www.bendlabs.com/.

[8] Bi, X., Moscovich, T., Ramos, G., Balakrishnan, R. and Hinckley, K. 2008. An exploration of pen rolling for pen-based interaction. Proceedings of the 21 st annual ACM symposium on User interface software and technology - UIST '08 
(New York, New York, USA, 2008), 191.

[9] Boem, A. and Troiano, G.M. 2019. Non-Rigid HCI: A review of deformable interfaces and input. DIS 2019 - Proceedings of the 2019 ACM Designing Interactive Systems Conference (New York, NY, USA, Jun. 2019), 885-906.

[10] Briotto Faustino, D., Nabil, S. and Girouard, A. Bend or PIN: Studying Bend Password Authentication with People with Vision Impairment. 2.

[11] Buxton, W., Hill, R. and Rowley, P. 1985. Issues and Techniques in TouchSensitive Tablet Input. Computer Graphics (ACM). 19, 3 (1985), 215-224. DOI:https://doi.org/10.1145/325165.325239.

[12] Cami, D., Matulic, F., Calland, R.G., Vogel, B. and Vogel, D. 2018. Unimanual Pen+Touch Input Using Variations of Precision Grip Postures. The 31st Annual ACM Symposium on User Interface Software and Technology - UIST '18 (New York, New York, USA, 2018), 825-837.

[13] Cherry, E. and Latulipe, C. 2014. Quantifying the creativity support of digital tools through the creativity support index. ACM Transactions on Computer-Human Interaction. 21, 4 (Aug. 2014), 1-25. DOI:https://doi.org/10.1145/2617588.

[14] Cho, Y., Bianchi, A., Marquardt, N. and Bianchi-Berthouze, N. 2016. RealPen: Providing realism in handwriting tasks on touch surfaces using auditory-tactile feedback. UIST 2016 - Proceedings of the 29th Annual Symposium on User Interface Software and Technology. (2016), 195-205. DOI:https://doi.org/10.1145/2984511.2984550.

[15] Ernst, M. and Girouard, A. 2016. Bending Blindly : Exploring Bend Gestures for the Blind. CHI'16 Extended Abstracts. (2016), 2088-2096. 
[16] Fares, E., Cheung, V. and Girouard, A. 2017. Effects of bend gesture training on learnability and memorability in a mobile game. Interactive Surfaces and Spaces. (2017), 240-245. DOI:https://doi.org/10.1145/3132272.3134142.

[17] Fellion, N., Eady, A.K. and Girouard, A. 2016. FlexStylus: A deformable stylus for digital art. SIGCHI Conference Extended Abstracts on Human Factors in Computing Systems (New York, New York, USA, 2016), 2482-2489.

[18] Fellion, N., Pietrzak, T. and Girouard, A. 2017. FlexStylus: Leveraging bend input for pen interaction. UIST 2017 - Proceedings of the 30th Annual ACM Symposium on User Interface Software and Technology (New York, NY, USA, Oct. 2017), $375-385$.

[19] Girouard, A., Lo, J., Riyadh, M., Daliri, F., Eady, A.K. and Pasquero, J. 2015. One-Handed Bend Interactions with Deformable Smartphones. Proceedings of the ACM CHI'15 Conference on Human Factors in Computing Systems. 1, (2015), 1509-1518. DOI:https://doi.org/10.1145/2702123.2702513.

[20] Grossman, T., Hinckley, K., Baudisch, P., Agrawala, M. and Balakrishnan, R. 2006. Hover widgets. Proceedings of the SIGCHI conference on Human Factors in computing systems - CHI '06 (New York, New York, USA, 2006), 861.

[21] Hasan, K., Yang, X.-D., Bunt, A. and Irani, P. 2012. A-coord input. Proceedings of the 2012 ACM annual conference on Human Factors in Computing Systems CHI '12 (New York, New York, USA, 2012), 805.

[22] Hinckley, K., Baudisch, P., Ramos, G. and Guimbretiere, F. 2005. Design and analysis of delimiters for selection-action pen gesture phrases in scriboli. $C H I$ 2005: Technology, Safety, Community: Conference Proceedings - Conference on 
Human Factors in Computing Systems. (2005), 451-460.

DOI:https://doi.org/10.1145/1054972.1055035.

[23] Hinckley, K., Chen, X. “Anthony” and Benko, H. 2013. Motion and context sensing techniques for pen computing. GI '13: Proceedings of the 2013 Graphics Interface Conference. (2013), 71-78.

[24] Hinckley, K., Guimbretiere, F., Agrawala, M., Apitz, G. and Chen, N. 2006. Phrasing techniques for multi-stroke selection gestures. Proceedings - Graphics Interface. 2006, (2006), 147-154.

[25] Hinckley, K., Wilson, A., Pahud, M., Benko, H., Irani, P., Guimbretière, F., Gavriliu, M., Chen, X. “Anthony” A., Matulic, F. and Buxton, W. 2014. Sensing techniques for tablet+stylus interaction. Proceedings of the 27th annual ACM symposium on User interface software and technology - UIST '14 (New York, New York, USA, 2014), 605-614.

[26] Hinckley, K., Yatani, K., Pahud, M., Coddington, N., Rodenhouse, J., Wilson, A., Benko, H. and Buxton, B. 2010. Pen + touch $=$ new tools. Proceedings of the 23nd annual ACM symposium on User interface software and technology - UIST '10 (New York, New York, USA, 2010), 27.

[27] Hung, M., Ledo, D. and Oehlberg, L. 2019. WatchPen: Using Cross-Device Interaction Concepts to Augment Pen-Based Interaction. Proceedings of the 21st International Conference on Human-Computer Interaction with Mobile Devices and Services - MobileHCI '19. (2019), 1-8.

DOI:https://doi.org/10.1145/3338286.3340122.

[28] Huot, S., Nancel, M. and Beaudouin-Lafon, M. 2008. PushMenu: Extending 
Marking Menus for Pressure-Enabled Input Devices.

[29] Kildal, J. 2012. Interacting with Deformable User Interfaces: Effect of Material Stiffness and Type of Deformation Gesture. Haptic and Audio Interaction Design. $71-80$.

[30] Kildal, J. and Wilson, G. 2012. Feeling it: the roles of stiffness, deformation range and feedback in the control of deformable ui. Proceedings of the 14th ACM international conference on Multimodal interaction - ICMI '12 (New York, New York, USA, 2012), 393.

[31] Kristensson, P. and Zhai, S. 2004. SHARK 2. Proceedings of the 17th annual ACM symposium on User interface software and technology - UIST '04 (New York, New York, USA, 2004), 43.

[32] Kurtenbach, G. and Buxton, W. 1993. The limits of expert performance using hierarchic marking menus. Conference on Human Factors in Computing Systems Proceedings. (1993), 482-487. DOI:https://doi.org/10.1145/169059.169426.

[33] Kurtenbach, G. and Buxton, W. 1994. User learning and performance with marking menus. Proceedings of the SIGCHI conference on Human factors in computing systems celebrating interdependence - CHI '94 (New York, New York, USA, 1994), 258-264.

[34] Lahey, B., Girouard, A., Burleson, W. and Vertegaal, R. 2011. PaperPhone: Understanding the Use of Bend Gestures in Mobile Devices with Flexible Electronic Paper Displays. Proc. CHI. Vancouver, (2011), 1303-1312. DOI:https://doi.org/10.1145/1978942.1979136.

[35] Lee, S.-S., Kim, S., Jin, B., Choi, E., Kim, B., Jia, X., Kim, D. and Lee, K. 2010. 
How users manipulate deformable displays as input devices. Proceedings of the 28th international conference on Human factors in computing systems - CHI '10 (New York, New York, USA, 2010), 1647.

[36] Lee, S., Lim, Y. and Lee, K.-P. 2012. Exploring the effects of size on deformable user interfaces. Proceedings of the 14th international conference on Humancomputer interaction with mobile devices and services companion - MobileHCI '12 (New York, New York, USA, New York, USA, 2012), 89.

[37] Lo, J. and Girouard, A. 2017. Bendy. Proceedings of the Tenth International Conference on Tangible, Embedded, and Embodied Interaction - TEI '17. (2017), 163-172. DOI:https://doi.org/10.1145/3024969.3024970.

[38] Lo, J. and Girouard, A. 2014. Fabricating bendy: Design and development of deformable prototypes. IEEE Pervasive Computing. 13, 3 (2014), 40-46. DOI:https://doi.org/10.1109/MPRV.2014.47.

[39] Martín-Gutiérrez, J. and Contero, M. 2011. FlexRemote: Exploring the Effectiveness of Deformable User Interface as an Input Device for TV. Springer Berlin Heidelberg.

[40] Matulic, F., Vogel, B., Kimura, N. and Vogel, D. 2019. Eliciting Pen-Holding Postures for General Input with Suitability for EMG Armband Detection. Proceedings of the 2019 ACM International Conference on Interactive Surfaces and Spaces (New York, NY, USA, Nov. 2019), 89-100.

[41] McGuffin, M., Burtnyk, N. and Kurtenbach, G. 2002. Fast sliders: Integrating marking menus and the adjustment of continuous values. Proceedings - Graphics Interface. (2002), 35-41. 
[42] Microsoft Surface Pro: https://www.microsoft.com/en-ca/p/surface-pro7/8n17j0m5zzqs?activetab $=$ overview.

[43] Murakami, T., Hayashi, K., Oikawa, K. and Nakajima, N. 1995. DO-IT:

Deformable Objects as Input Tools. Conference companion on Human factors in computing systems - CHI '95 (New York, New York, USA, 1995), 87-88.

[44] Ortega, M. and Goguey, A. 2019. BEXHI: A Mechanical Structure for Prototyping Bendable and EXpandable Handheld Interfaces. Proceedings of the 2019 ACM International Conference on Interactive Surfaces and Spaces (New York, NY, USA, Nov. 2019), 269-273.

[45] Ramos, G., Boulos, M. and Balakrishnan, R. 2004. Pressure widgets. Proceedings of the 2004 conference on Human factors in computing systems - CHI '04 (New York, New York, USA, 2004), 487-494.

[46] Ramos, G.A. and Balakrishnan, R. 2007. Pressure marks. Conference on Human Factors in Computing Systems - Proceedings. (2007), 1375-1384.

DOI:https://doi.org/10.1145/1240624.1240834.

[47] Ryokai, K., Marti, S. and Ishii, H. 2004. I/O Brush: Drawing with everyday objects as ink. Proceedings of the 2004 conference on Human factors in computing systems - CHI '04 (New York, New York, USA, 2004), 303-310.

[48] Senturia, S. 2002. Microsystem Design. Kluwer Academic Publishers.

[49] Shahmiri, F. and Dietz, P.H. 2020. ShArc : A Geometric Technique for MultiBend / Shape Sensing. (2020), 1-12.

[50] Shinohara, A., Shinohara, A., Tomita, J., Tomita, J., Kihara, T., Kihara, T., Nakajima, S., Nakajima, S., Ogawa, K. and Ogawa, K. 2007. Stylus Enhancement 
to Enrich Interaction with Computers. Springer Berlin Heidelberg.

[51] Shorey, P. and Girouard, A. 2017. Bendtroller: An exploration of in-game action mappings with a deformable game controller. SIGCHI Conference on Human Factors in Computing Systems (2017), 1447-1458.

[52] Soft Angular Displacement Sensor Theory Manual: https://github.com/bendlabs/two_axis_ads.

[53] Song, H., Benko, H., Guimbretiere, F., Izadi, S., Cao, X. and Hinckley, K. 2011. Grips and gestures on a multi-touch pen. Proceedings of the 2011 annual conference on Human factors in computing systems - CHI '11 (New York, New York, USA, 2011), 1323.

[54] Strohmeier, P., Burstyn, J., Carrascal, J.P., Levesque, V. and Vertegaal, R. 2016. ReFlex: A flexible smartphone with active haptic feedback for bend input. TEI 2016 - Proceedings of the 10th Anniversary Conference on Tangible Embedded and Embodied Interaction (New York, New York, USA, 2016), 185-192.

[55] Tan, H.Z., Srinivasan, M.A., Eberman, B. and Cheng, B. 1994. Human Factors for the Design of Force-Reflecting Haptic Interfaces. Dynamics Systems and Control. 55,1 (1994).

[56] Tian, F., Xu, L., Wang, H., Zhang, X., Liu, Y., Setlur, V. and Dai, G. 2008. Tilt menu. Proceeding of the twenty-sixth annual CHI conference on Human factors in computing systems - CHI '08 (New York, New York, USA, 2008), 1371.

[57] Tian, F., Xu, L., Wang, H., Zhang, X., Liu, Y., Setlur, V., Dai, G., Road, P.M. and Alto, P. 2008. Tilt Menu : Using the 3D Orientation Information of Pen Devices to Extend the Selection Capability of Pen-based User Interfaces. Conference on 
Human Factors in Computing Systems - Proceedings. (2008), 1371-1380.

DOI:https://doi.org/10.1145/1357054.1357269.

[58] Vogel, D. and Casiez, G. 2011. Conté: Multimodal input inspired by an artist's crayon. Proceedings of the 24th annual ACM symposium on User interface software and technology - UIST '11 (New York, New York, USA, 2011), 357.

[59] Wightman, D., Ginn, T. and Vertegaal, R. 2010. TouchMark: Flexible document navigation and bookmarking techniques for E-book readers. Proceedings of Graphics Interface 2010. Canadian Information Processing Society. (2010), 241244.

[60] Xin, Y., Bi, X. and Ren, X. 2011. Acquiring and pointing. Proceedings of the 2011 annual conference on Human factors in computing systems - CHI '11 (New York, New York, USA, 2011), 849.

[61] Xin, Y., Bi, X. and Ren, X. 2012. Natural use profiles for the pen. Proceedings of the 2012 ACM annual conference on Human Factors in Computing Systems - CHI '12 (New York, New York, USA, 2012), 801.

[62] Youssefi, K. and Anagnous, T. Structures and Stiffness.

[63] Zhao, S., Agrawala, M. and Hinckley, K. 2006. Zone and polygon menus. Proceedings of the SIGCHI conference on Human Factors in computing systems CHI '06 (New York, New York, USA, 2006), 1077.

[64] Zhou, X. and Ren, X. 2009. A comparison of pressure and tilt input techniques for cursor control. IEICE Transactions on Information and Systems. E92-D, 9 (2009), 1683-1691. DOI:https://doi.org/10.1587/transinf.E92.D.1683. 
Appendices 


\section{Appendix A}

\section{A.1 Consent Form}

Carleton

Canada's Capital University
Permission to take part in a research study:

\section{Evaluating Bendable Inputs for Digital Stylus} Interactions

\author{
CUREB-B Clearance \# 11345
}

Date of ethics clearance: Aug. $22^{\text {nd }}, 2019$

Clearance for the Collection of Data Expires: Aug. 31 $31^{\text {st }}, 2020$

What we are studying: This study explores bendable interactions of a digital bendable stylus. The researchers are Alfrancis Guerrero in the School of Information Technology at Carleton University, Supervised by Dr. Audrey Girouard. The study will last 60 minutes. Participants will be asked to use a digital bendable stylus and perform a series of tasks that utilize bendable interactions. The tasks instruct the participant to use the bend or pressure stylus to change the brush stroke width and move along a path. They will be asked to complete a post questionnaire following completion of the study.

To participate you must be at least 18 years old, able to provide informed consent, and be able to operate a smartphone with your hands.

We will record: Images of the prototype in use and your think aloud statements.

We will not record: Your face, name, or other identifying material.

Withdrawing from the study: You have the right to end your participation at any time during the session for any reason, simply inform the researcher. If you choose to withdraw, we will stop collecting data and any information gathered will be destroyed and not used in the research. It is not possible to withdraw after the study session has ended because we do not associate your identity with collected data.

Video recording: With your consent, a video recording device will be used to capture device interactions and your statements during the study. Images and video stills may be used in promotions and publications. We will crop or blur images to remove faces in publications, but there is still an inherent risk that you may be identifiable in such imagery. You do not have to consent to video recording to participate in the study.

Participant data protection and use: Research data collected during the session and in surveys will not be associated with your identity. Stored research data will be password protected. Hard copies will be kept in a locked cabinet at Carleton University. Research data will be accessible to the researchers and the research supervisor.

Once the project is completed, research data will be kept to inform future research. | 
If you would like a copy of the finished research project, you are invited to contact the researcher to request an electronic copy which will be provided to you.

Compensation: You will receive $\$ 15.00$ in cash upon completion of the study, which should last approximately $60 \mathrm{~min}$. This compensation will still be provided if you elect to withdraw from the study.

Your Consent:

Do you agree to participate in the study:

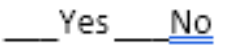

Do you agree to be video-recorded:

_ Yes__ No

Signature of participant

Date

Siqnature of researcher

Date

Researchers:

Alfrancis Guerrero

Information Technology

Carleton University

Alfrancis.Guerrero@carleton.ca

\[ \frac{\text { Date }}{\text { Dr.|Audrey Girouard (Research Supervisor) }} \]
Information Technology
Carleton University
Audrey.Girouard@carleton.ca

The ethics protocol for this project was reviewed by the Carleton University Research Ethics Board CUREB-B \#11345, which provided clearance to carry out the research. If you have any ethical concerns with the study, please contact Ethics Board Chair, Carleton University Research Ethics Board-B (phone 613-520-2600 ext. 4085 or via email at ethics@carleton.ca). 


\section{Appendix B}

\section{B.1 Study 1 \& 2 Demographics Questionnaire}

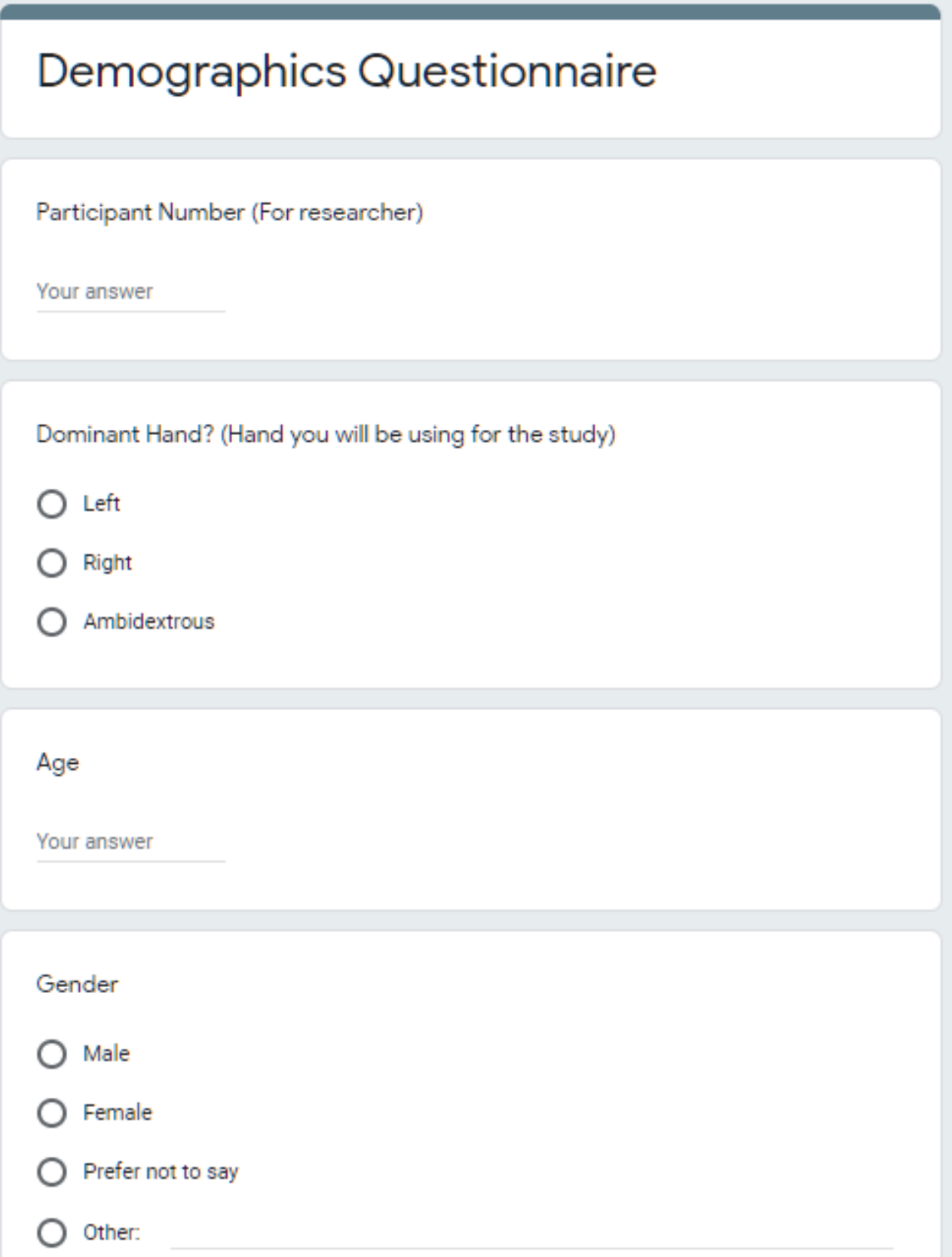


Status

Student

Employed

Other:

Previous experience with deformable/bendable interactions

None

Some Experience

Extensive Experience

Previous experience with using pens/styluses

None

Some Experience

Extensive Experience 


\section{Digital Stylus Usage Questionnaire}

How often do you use a digital stylus on a touch device to do the following...

Drawing

Never

Occasionally

Sometimes

Often

Always

Digital Writing

Never

Occasionally

Sometimes

often

Always

Editing Digital Documents

Never

Occasionally

Sometimes

Often

Always 
Regular Stylus Usage Questionnaire

How often do you use REGULAR STYLUS (pencil or pen) on a piece of paper

Drawing

Never

Occasionally

Sometimes

Often

Always

Writing

Never

Occasionally

Sometimes

$\mathrm{O}$ often

Always 


\section{B.2 Between Task Questionnaire}

\section{Task Questionnaire (For Participant)}

The brushstroke width changed as I intended

Strongly Agree

Agree

Neutral

Disagree

Strongly Disagree

The brushstroke width was responsive to my inputs
Strongly Agree
Agree
Neutral
Disagree
Strongly Disagree

I was able to navigate to the proper target width
Strongly Agree
Agree
Neutral
Disagree
Strongly Disagree 


\section{B.3 Post Questionnaire}

\section{Post Questionnaire (After Study has been completed)}

Rank the Flexbility from most preferred to least preferred (Soft, Medium, Hard)

ie. Soft, Medium, Hard

Your answer

What is the reasoning behind your rankings?

Your answer

The best part of using a bendable stylus was...

Your answer

The worst part of using a bendable stylus was...

Your answer

The best part of using a pressure stylus was...

Your answer

The worst part of using a pressure stylus was...

Your answer 
Which stylus input did you prefer?

\section{Flexible}

Pressure

Why?

Your answer

Any additional comments

Your answer 


\section{B.4 Creative Survey Index Questionnaires}

I would be happy to use this system or tool on a regular basis.

$\begin{array}{lllllllllllll} & 1 & 2 & 3 & 4 & 5 & 6 & 7 & 8 & 9 & 10 & \\ \text { Highly Disagree } & \bigcirc & \bigcirc & \bigcirc & \bigcirc & \bigcirc & \bigcirc & \bigcirc & \bigcirc & \bigcirc & \bigcirc & \text { Highly Agree }\end{array}$

It was easy for me to explore many different ideas, options, designs, or outcomes, using this system or tool.
$\begin{array}{llllllllll}1 & 2 & 3 & 4 & 5 & 6 & 7 & 8 & 9 & 10\end{array}$
Highly Disagree $\mathrm{O} 0 \mathrm{O} 0 \mathrm{O} 0 \mathrm{O}$ Highly Agree

I became so absorbed in the activity that I forgot about the system or tool that I was using.

$\begin{array}{lllllllllllll} & 1 & 2 & 3 & 4 & 5 & 6 & 7 & 8 & 9 & 10 & \\ \text { Highly Disagree } & \mathrm{O} & \mathrm{O} & \mathrm{O} & \mathrm{O} & \bigcirc & \bigcirc & \bigcirc & \bigcirc & \bigcirc & \bigcirc & \text { Highly Agree }\end{array}$

I was satisfied with what I got out of the system or tool.

$\begin{array}{lllllllllllll} & 1 & 2 & 3 & 4 & 5 & 6 & 7 & 8 & 9 & 10 & \\ \text { Highly Disagree } & \bigcirc & \bigcirc & \bigcirc & \bigcirc & \bigcirc & \bigcirc & \bigcirc & \bigcirc & \bigcirc & \bigcirc & \text { Highly Agree }\end{array}$

I enjoyed using the system or tool.

$\begin{array}{llllllllllllll} & 1 & 2 & 3 & 4 & 5 & 6 & 7 & 8 & 9 & 10 & \\ \text { Highly Disagree } & \bigcirc & \bigcirc & \bigcirc & \bigcirc & \bigcirc & \bigcirc & \bigcirc & \bigcirc & \bigcirc & \bigcirc & \text { Highly Agree }\end{array}$ 
The system or tool was helpful in allowing me to track different ideas. outcomes,or possibilities.

$\begin{array}{llllllllllllll} & 1 & 2 & 3 & 4 & 5 & 6 & 7 & 8 & 9 & 10 & \\ \text { Highly Disagree } & 0 & \bigcirc & \bigcirc & \bigcirc & \bigcirc & \bigcirc & \bigcirc & \bigcirc & \bigcirc & \bigcirc & \text { Highly Agree }\end{array}$

The system or tool allowed me to be very expressive.

$\begin{array}{lllllllllllll} & 1 & 2 & 3 & 4 & 5 & 6 & 7 & 8 & 9 & 10 \\ \text { Highly Disagree } & \bigcirc & \bigcirc & \bigcirc & \bigcirc & \bigcirc & \bigcirc & \bigcirc & \bigcirc & \bigcirc & \bigcirc & \text { Highly Agree }\end{array}$

My attention was fully tuned to the activity, and I forgot about the system or tool that I was using.

$\begin{array}{llllllllllllll} & 1 & 2 & 3 & 4 & 5 & 6 & 7 & 8 & 9 & 10 & \\ \text { Highly Disagree } & \bigcirc & \bigcirc & \bigcirc & \bigcirc & \bigcirc & \bigcirc & \bigcirc & \bigcirc & \bigcirc & \bigcirc & \text { Highly Agree }\end{array}$

I was able to be very creative while doing the activity inside this system or tool.

$\begin{array}{llllllllllllll} & 1 & 2 & 3 & 4 & 5 & 6 & 7 & 8 & 9 & 10 & \\ \text { Highly Disagree } & \mathrm{O} & \bigcirc & \bigcirc & \bigcirc & \bigcirc & \bigcirc & \bigcirc & \bigcirc & \bigcirc & \bigcirc & \text { Highly Agree }\end{array}$

What I was able to produce was worth the effort I had to exert to produce it.
$\begin{array}{llllllllll}1 & 2 & 3 & 4 & 5 & 6 & 7 & 8 & 9 & 10\end{array}$
Highly Disagree $\mathrm{O} O \mathrm{O} O \mathrm{O} O \mathrm{O}$ Highly Agree 


\section{Appendix C}

\section{C.1 Participant Drawings with HyperBrush}
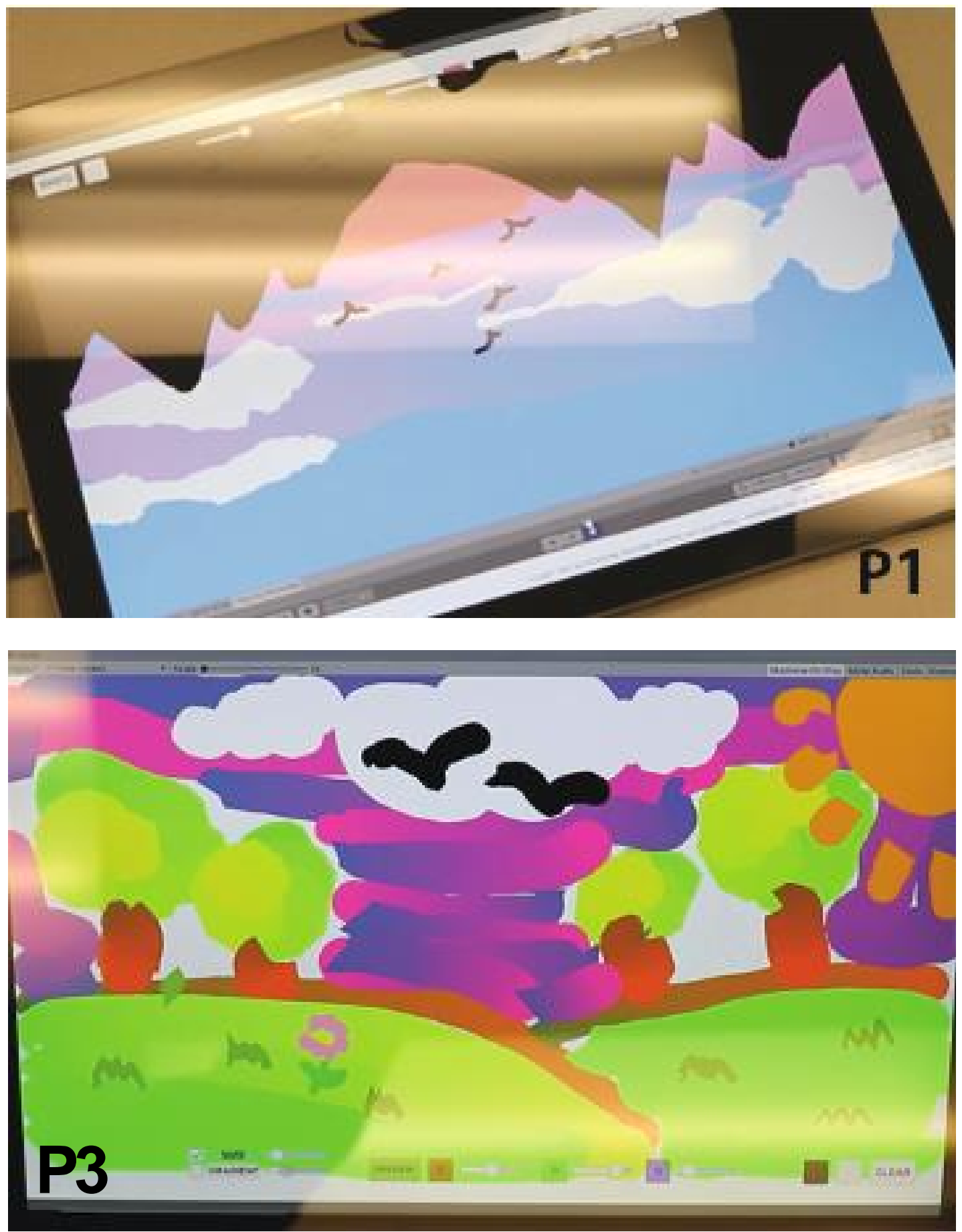

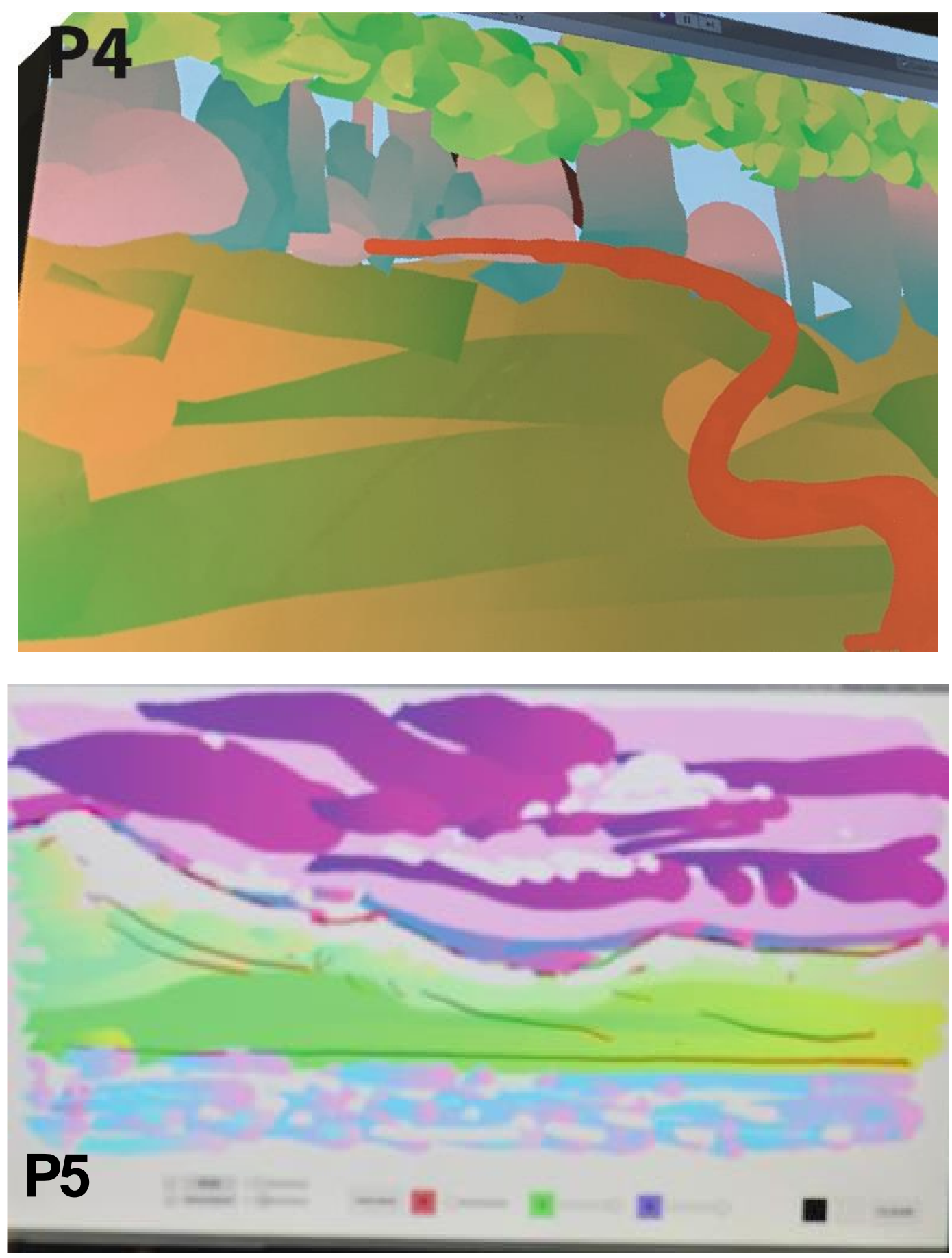

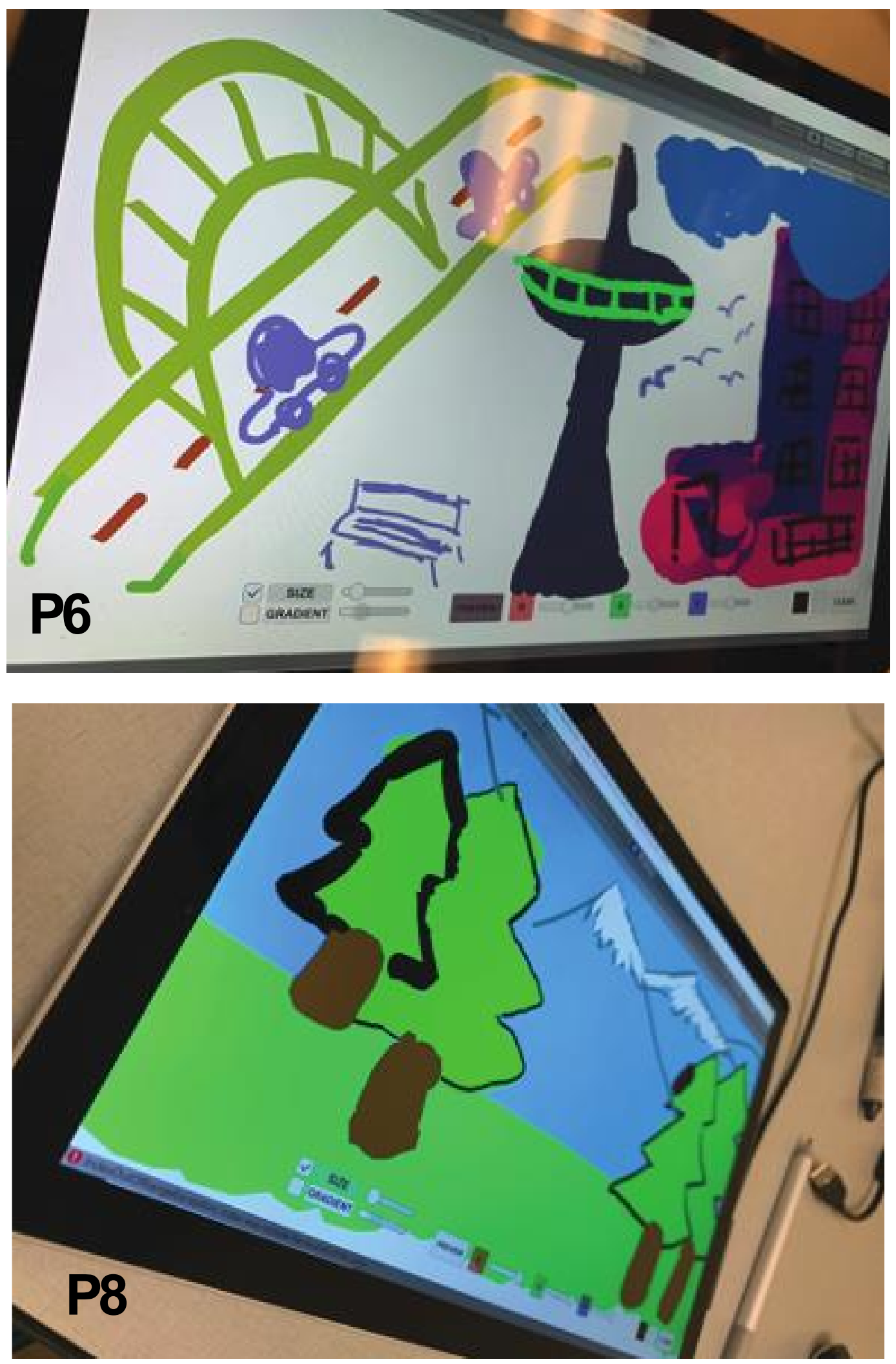


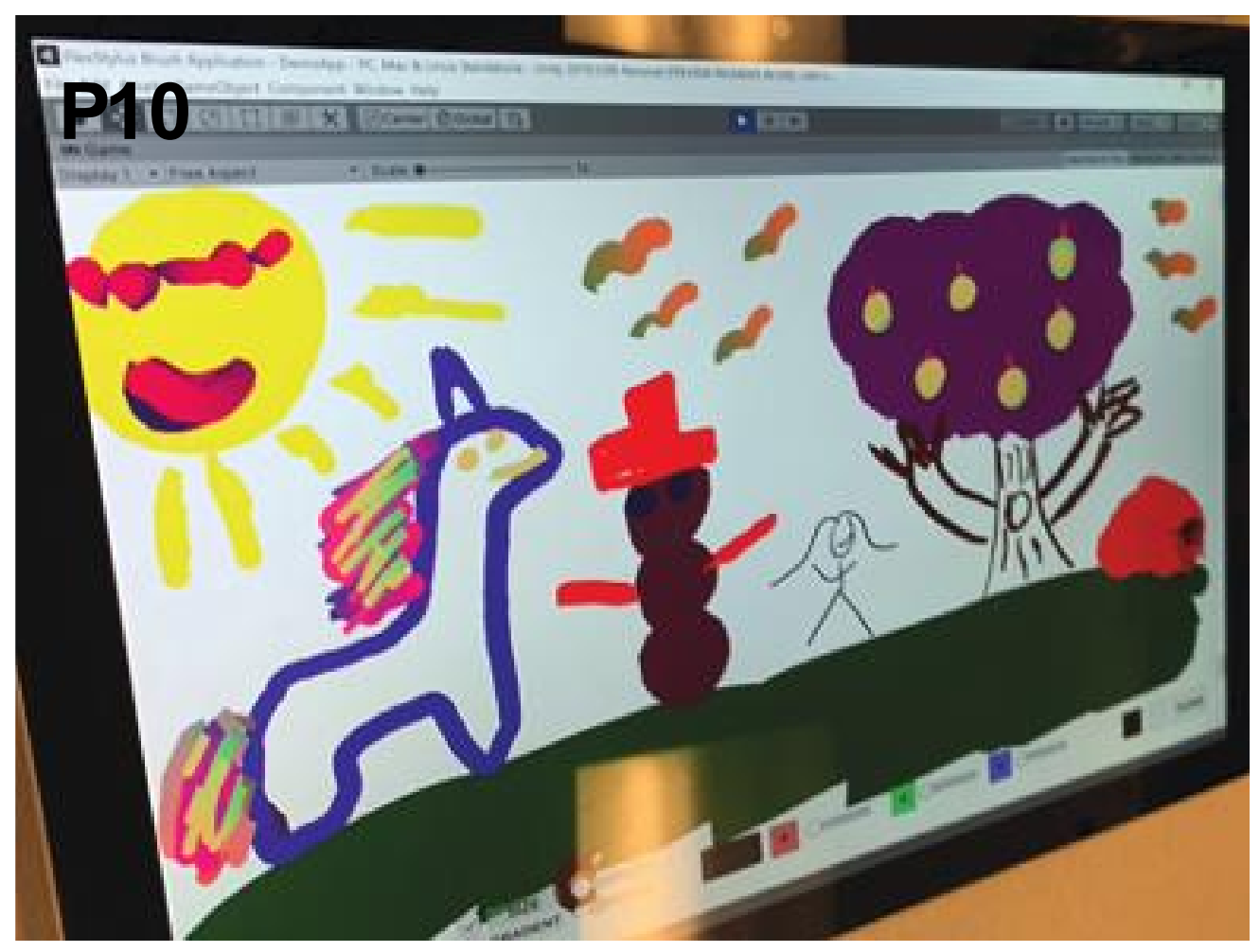

C.2 Participant Drawings with Pressure Pen

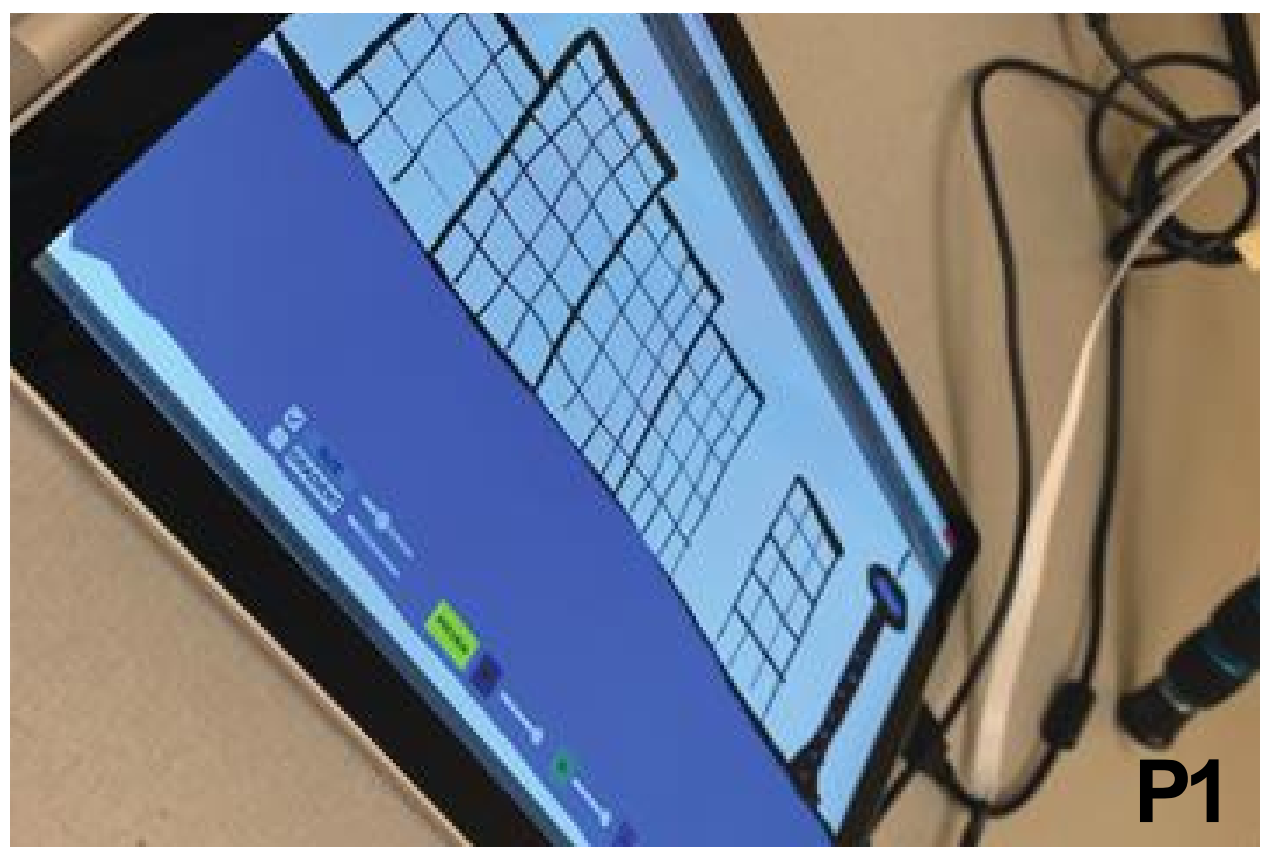



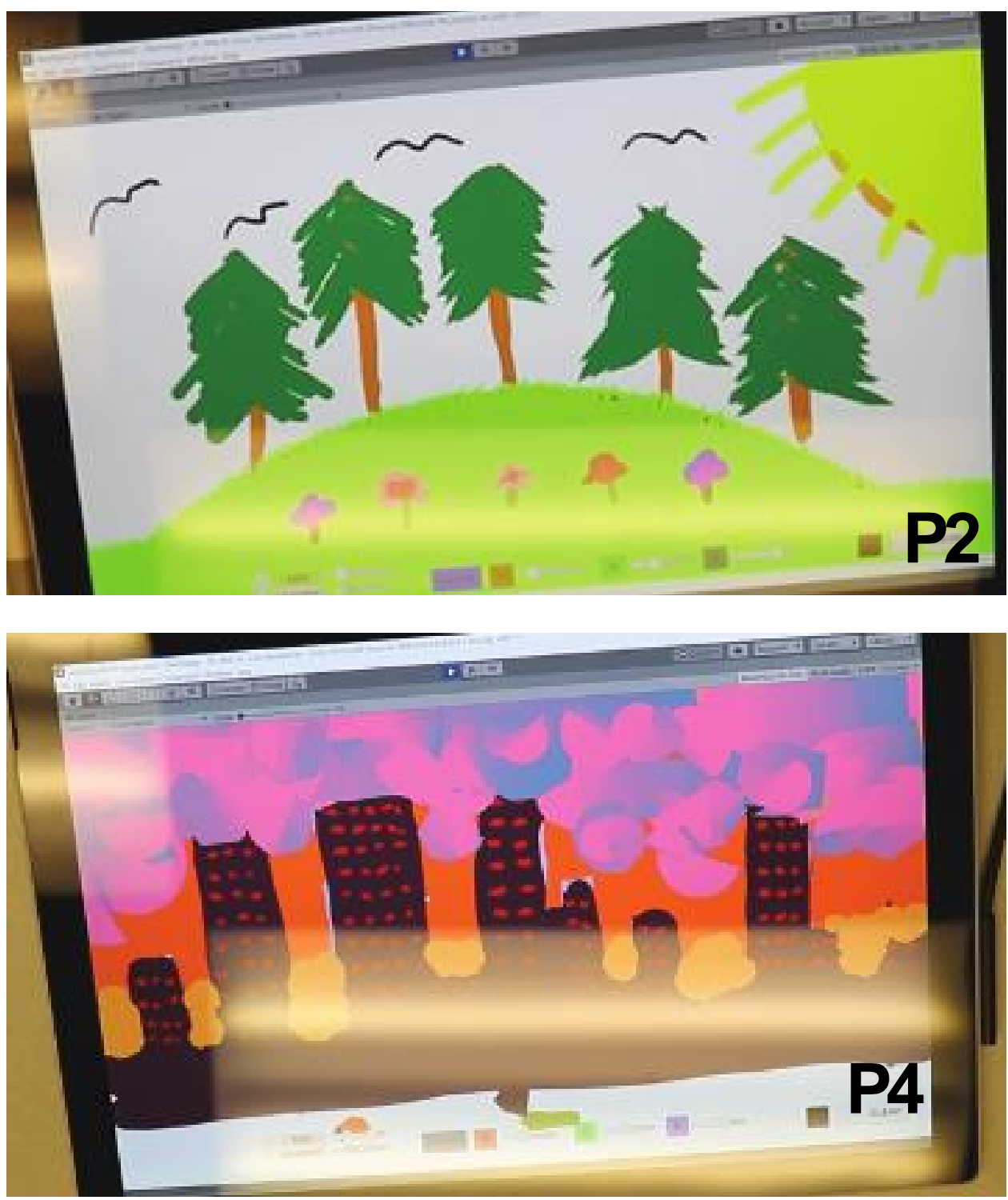

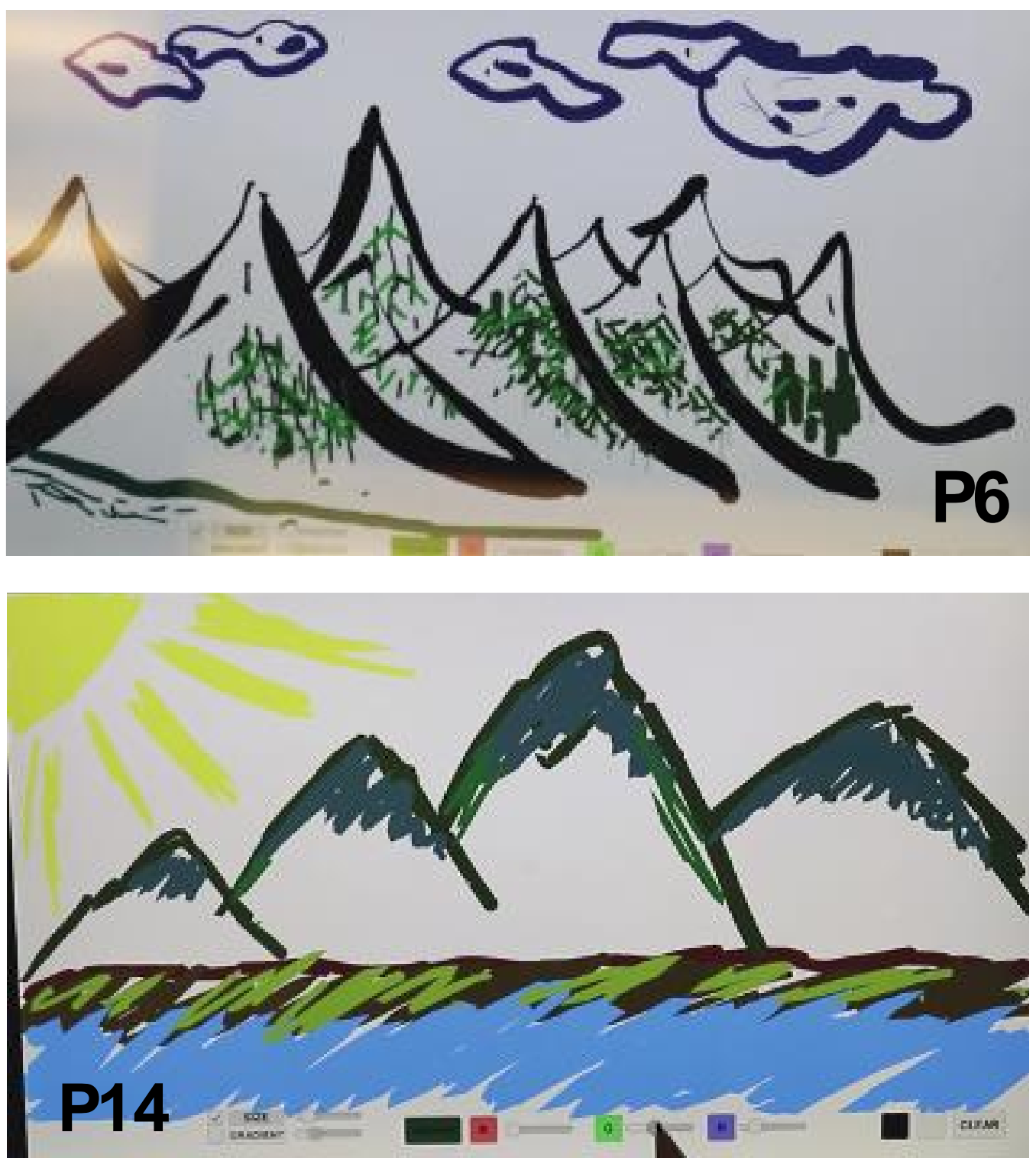

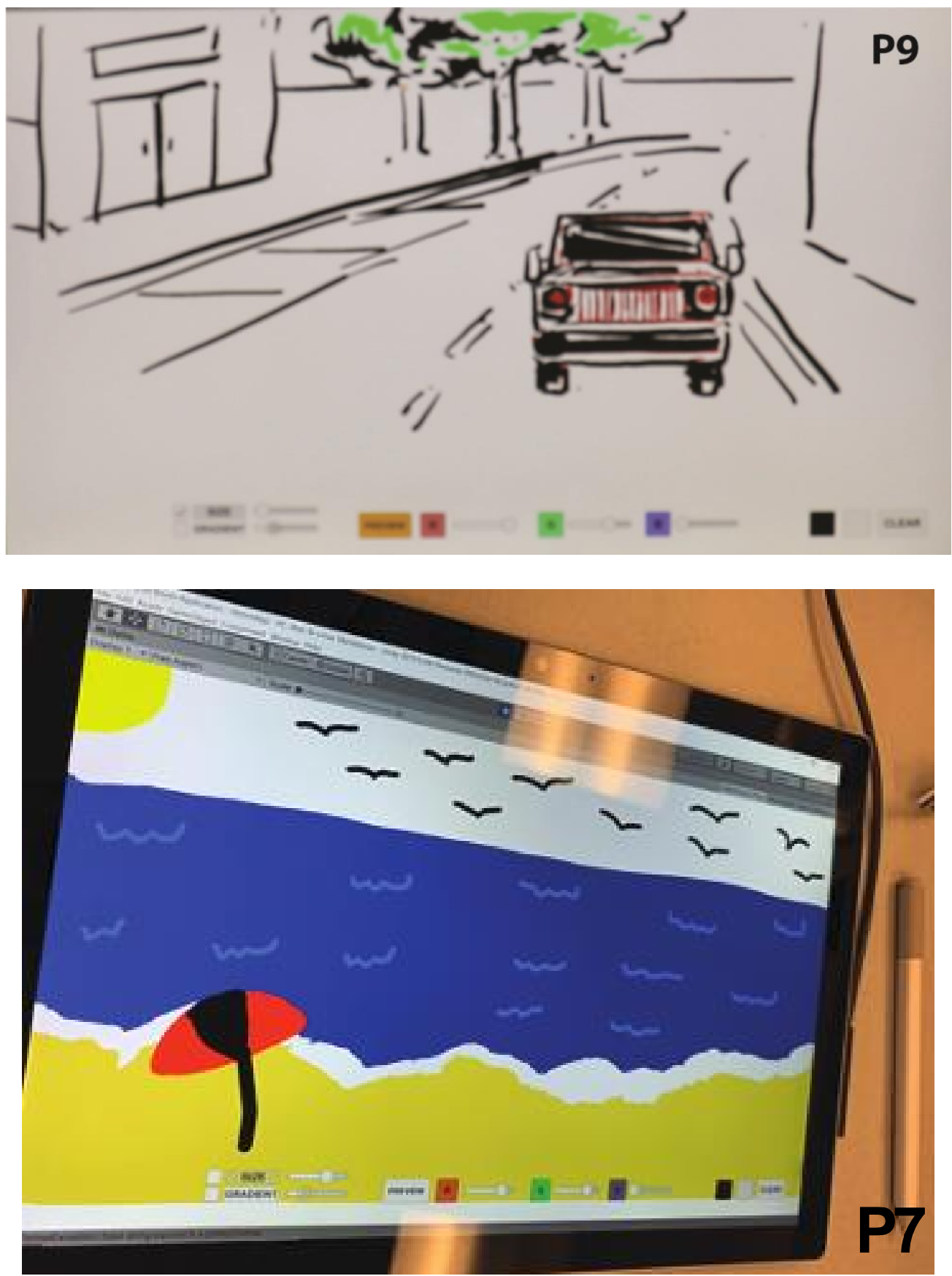

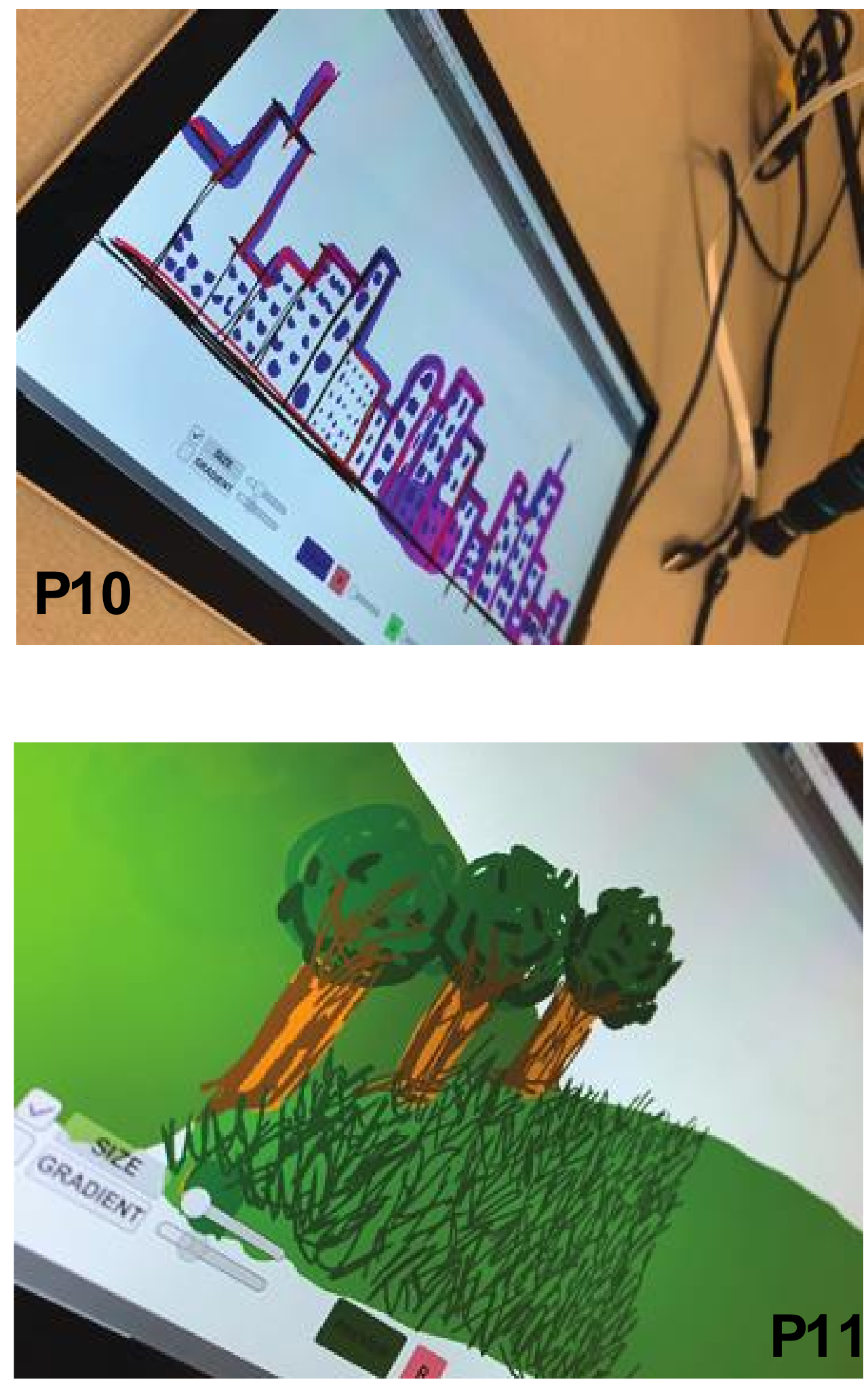